\title{
Broadband stripline ferromagnetic resonance spectroscopy of ferromagnetic films, multilayers and nanostructures
}

\author{
Ivan S. Maksymov and Mikhail Kostylev ${ }^{\mathrm{a}}$ \\ School of Physics M013, University of Western Australia, Crawley 6009, WA, Australia \\ ${ }^{a}$ Corresponding author, e-mail: Mikhail.Kostylev@uwa.edu.au
}

\begin{abstract}
This article presents a comprehensive critical overview of fundamental and practical aspects of the modern stripline broadband ferromagnetic resonance (BFMR) spectroscopy largely employed for the characterisation of magnetic low-dimensional systems, such as thin ferro- and ferromagnetic, multiferroic and half-metallic films, multi-layers and nanostructures. These planar materials form the platform of the nascent fields of magnonics and spintronics. Experimental and theoretical results of research on these materials are summarised, along with systematic description of various phenomena associated with the peculiarities of the stripline BFMR, such as the geometry of stripline transducers, the orientation of the static magnetic field, the presence of microwave eddy currents, and the impacts of non-magnetic layers, interfaces and surfaces in the samples. Results from more than 240 articles, textbooks and technical reports are presented and many practical examples are discussed in detail. This review will be of interest to both general physical audience and specialists conducting research on various aspects of magnetisation dynamics and nanomagnetism.
\end{abstract}

Keywords: ferromagnetic resonance, spin waves, eddy currents, thin films, nanostructures, metamaterials

\section{Introduction}

Much of the modern technology that energises today's society is based on magnetism. Magnets and numerous sophisticated magnetic effects play key roles in computer hard drives, medical equipment, telecommunications systems, data storage, sensors [1], and non-volatile random access memory already used in spaceships [2]. However, modern magnets are not only bulk magnets familiar from high-school physics classes. A large class of magnetic devices is based on low-dimensional systems, such as thin continuous films or complex nano-patterned structures. This is because thin magnetic films and nanostructures may possess very different properties with respect to their counterpart bulk materials, in part due to the presence of surfaces and interfaces. At a film surface (or interface) the symmetry is lower than in the bulk of the film, and the atoms experience a different local environment. When the films are made sufficiently thin, the impact of material boundaries, surfaces and interfaces becomes significant. From the technological perspective this is extremely important because by customising multi-layered thin films and nanostructures it is possible to create materials with unique properties that do not exist in nature.

The study of magnetic thin films has been around for nearly seven decades (see, e.g., [3, 4, $5,6,7,8,9,10])$ and this research direction still remains very active. An important aspect of the current research is the microwave magnetisation dynamics in thin films, multi-layers, and planar nanostructures made from ferromagnetic metallic materials. The interest in these structures is motivated by their potential as a future platform for microwave signal processing $[11,12,13,14,15$, 16], magnetic logics [17, 18, 19, 20, 21], magnetic memory [22, 23, 24, 25, 26], sensors [27, 28, 29, 30, 
$31]$, and other areas of science and technology [1].

Recently, it has been demonstrated that the magnetisation of a magnetic material can be controlled by using electric currents that transport spin angular momentum [32]. A changing magnetisation orientation produces currents that transport spin angular momentum. Understanding how these processes occur reveals the intricate connection between the magnetisation and the spin transport, and lays foundations of a new technology called spintronics. This technology can be used to develop novel devices that generate, store, or processes information via the magnetisation direction (spin orientation in quantum language) [33]. Consequently, there is a huge interest in a number of spintronic effects such as the spin transfer torque, direct and inverse Spin-Hall effects, and spin pumping [33, 34, 35]. A deep understanding of physics of these effects is crucial for the development of magnetic random access memory (MRAM), spin-torque MRAM, and spin-torque nano-oscillators $[33,34]$. The time scale for responses of the aforementioned devices corresponds to the microwave frequency range. These devices are based on continuous thin films or nanostructures consisting of an (often intricate) sequence of thin non-magnetic and ferromagnetic layers. Furthermore, we are now witnessing a huge progress in the microwave quality of halfmetallic [36] and ferrimagnetic films [37,38] with thicknesses in the nanometre range. It is highly likely that availability of these new materials will give a new boost to this research direction.

More generally, the advances in the research on magnetisation dynamics and spintronics stem from the progress in nanofabrication and experimental characterisation techniques. One of the key characterisation techniques - the broadband ferromagnetic resonance (FMR) spectroscopy - is the main subject of this review. For several decades the FMR spectrometers have employed a microwave cavity to take a measurement of FMR absorption [39, 40]. The importance of the FMR technique has recently skyrocketed thanks to the advent of the broadband stripline FMR (BFMR) spectroscopy. The BFMR allows characterising materials in a broad range of frequencies, often from several hundreds of $\mathrm{MHz}$ to $30-40 \mathrm{GHz}$ [41, 42]. This allowed significant improvement of accuracy of extraction of material parameters from the raw FMR absorption traces [42].

Furthermore, the Gilbert magnetic damping constant [43] is extracted as a slope of the frequency dependence of the resonance line width. Without BFMR it is difficult to distinguish between the contribution to the resonance linewidth due to the intrinsic damping (given by the value of the Gilbert constant) and extrinsic contributions [44, 45, 46]. The high interest in the magnetisation damping originates, for example, from the technological importance of the spin transfer and spin pumping effects (see, e.g., [32, 33, 34, 35, 41, 42, 47]). In this case, the Gilbert damping constant affects the critical current needed to switch the magnetisation direction [32, 48]. In the case of self-sustained oscillations, the Gilbert damping affects the instability current that confines the regions of microwave emission [49, 50, 51].

We also stress the importance of the BFMR spectroscopy in the emerging area of magnetically tuneable microwave meta-materials and sensors of various substances including sensing of gases and nanoparticles. For more information, we refer the interested reader to Ref. [29, $52,53,54,55,56,57]$ and references therein.

Several reviews, books chapters, and technical reports on various aspects of the FMR spectroscopy have been completed up to date $[10,39,58,59,60,61,62,63,64]$. However, some of these works are either technically outdated [10], written in very introductory style [61, 64] or very specialised and focused on technical aspects of particular FMR setups [39, 58, 59, 62, 63] and their particular applications, e.g., on the characterisation of materials for high-frequency signal 
processing devices [62]. Despite a high practical value of the previous attempts to overview FMR spectroscopy, there is obviously a lack of a comprehensive review of the stripline BFMR spectroscopy. In order to be really useful for the research community, the scope of such a review should go beyond just giving a bird's-eye view of various aspects of the BFMR spectroscopy. It should also discuss the important peculiarities of the BFMR, the practical methods of raw data analysis and interpretation, as well as the numerical methods helping to better understand experimental results. The aim of the present review is to fill this gap.

Before we start, we note that results presented in many articles on FMR measurements and relevant topics are given in Gaussian units based on the cgs system. Furthermore, in many modern articles Gaussian units co-exist with SI units because, for example, the Oersted units are often used to display the applied field in raw data measurements. For this reason, below the reader will find that some quantities are expressed in cgs units and some in SI ones. We refer the reader to the magnetic units conversion table given in Appendix I. We also recommend Ref. [65] containing useful formulae in both systems of units.

\section{Ferromagnetic resonance spectroscopy}

\subsection{Ferromagnetic resonance and standing spin-wave modes}

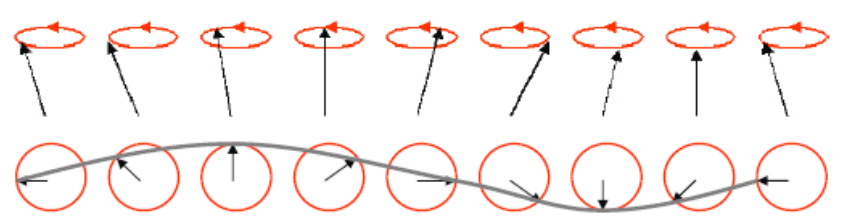

FIG. 1. A spin wave on a spin chain. Top: the spins viewed in perspective. Bottom: the spins viewed from above, showing one wavelength. The wave is drawn through the ends of the spin vectors. For clarity, the trajectories of spin precession are shown as circles.

Central for the understanding of the microwave magnetisation dynamics and the ferromagnetic resonance experiment is the notion of spin waves. Spin waves are eigen-excitations in ferromagnetic media, existing in the microwave frequency range. Spin waves represent collective precessional motion of spins coupled by short-range exchange and long-range dipole interactions in a magnetic medium (Fig. 1). The classical description of spin waves is given by the LandauLifshitz-Gilbert (LLG) equation [43] for the magnetisation vector $\mathbf{M}$

$$
\frac{\partial \mathbf{M}}{\partial t}=\gamma\left(\mathbf{M} \times \mathbf{H}_{\mathrm{eff}}\right)+\frac{\alpha_{\mathrm{G}}}{M_{\mathrm{s}}}\left(\mathbf{M} \times \frac{\partial \mathbf{M}}{\partial t}\right),
$$

where $\gamma$ is the gyromagnetic ratio, $\mathbf{H}_{\mathrm{eff}}$ is the effective magnetic field inside the medium, $M_{\mathrm{s}}$ is the saturation magnetisation, and $\alpha_{\mathrm{G}}$ is the Gilbert damping coefficient. The first term on the righthand-side (r.h.s) of Eq. (1) gives rise to the precessional motion of the magnetisation vector about an equilibrium direction determined by the effective magnetic field. The second term is the damping term responsible for the magnetisation vector spiralling back to the static equilibrium. Equation (1) can be solved together with the Maxwell's equations for particular geometries to yield spin wave 
eigen-modes. The eigen-frequencies depend on the sample shape, external field and material parameters.

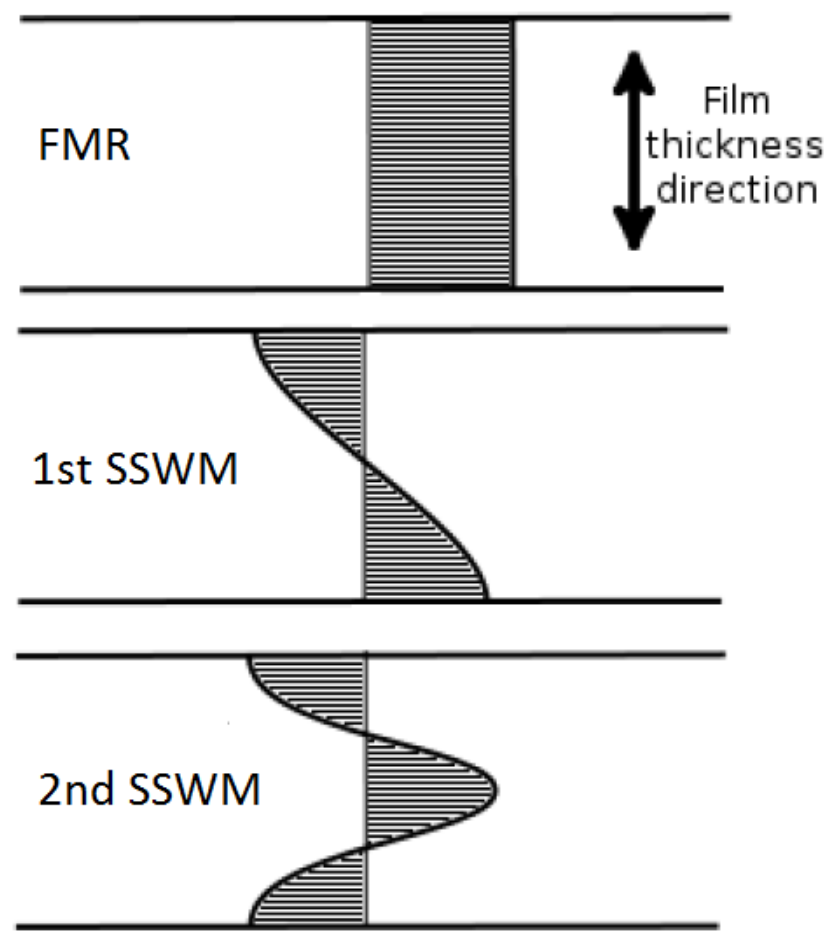

FIG. 2. Schematics of the spin precession amplitude across the film thickness direction for the first three standing spin wave modes (SSWMs). These profiles assume that the magnetisation vector is unpinned at the film surfaces. The fundamental resonance mode (FMR mode) represents in-phase precession with uniform amplitude across the thickness direction, while for the ${ }^{\text {st }}$ SSWM the spins at the top and bottom precess in anti-phase.

Ferromagnetic resonance (FMR) - also known as the fundamental mode of uniform precession of magnetisation - is the case where the spins precess with the same phase and amplitude over the whole volume of the magnetic material (Fig. 2). It may be considered as a spin wave with an infinite wavelength or zero wave number. FMR was unknowingly discovered by V. K. Arkad'yev in 1911 [66] - he was first to observe resonant absorption of ultra-high frequency radiation by ferromagnetic materials. A qualitative explanation of FMR along with an explanation of the results by Arkad'yev was given by J. Dorfmann in 1923; he suggested that the optical transitions due to Zeeman splitting could provide a way to study ferromagnetic structure [67].

The frequency of the uniform FMR mode for an ellipsoid of revolution magnetised along one of its axis (z-direction) is given by the Kittel equation [43]

$$
f^{2}=\gamma^{2}\left(H_{\mathrm{res}}+\left(N_{x x}-N_{z z}\right)\right)\left(H_{\mathrm{res}}+\left(N_{y y}-N_{z z}\right)\right),
$$

where $N_{\mathrm{xx}}, N_{\mathrm{yy}}$, and $N_{\mathrm{zz}}$ are the demagnetising factors along the axes of the ellipsoid.

In the limiting case of a thin continuous film magnetised in its plane (IP) $\left(N_{\mathrm{xx}}=1\right.$, $\left.N_{\mathrm{yy}}=N_{\mathrm{zz}}=0\right)$ this formula reduces to [43]

$$
f^{2}=\gamma^{2} H_{\text {res }}\left(H_{\text {res }}+4 \pi M_{s}\right) \text {, }
$$


and for the case of magnetisation of the film perpendicular to its plane (PP, $\left.N_{z z}=1\right)$ one has

$$
f=\gamma\left(H_{\text {res }}-4 \pi M_{s}\right) .
$$

In Eqs. (2-4), $f$ is the resonant frequency and $H_{\text {res }}$ is the resonant magnetic field. This mode is efficiently excited if the microwave magnetic field of the driving source is uniform across the thickness of the film [68].

Besides the uniform (or often quasi-uniform) mode, confined geometries often support excitation of higher-order FMR modes which represent standing spin waves across the direction of confinement ["Standing Spin Wave Modes" (SSWM)]. As the name implies, the dynamic magnetisation profiles of SSWMs across the thickness of the film represent stationary waves with wave vectors $k=n \pi / d$, where $n$ is the mode number and $d$ is the thickness of the film (Fig. 2). The IP Kittel equation is then modified [43]

$$
f^{2}=\gamma^{2}\left(H_{\mathrm{res}}+H_{e x}\right)\left(H_{\mathrm{res}}+H_{e x}+4 \pi M_{s}\right),
$$

where $H_{\mathrm{ex}}=D k^{2}$ is the exchange field, and $D$ is the exchange stiffness constant.

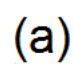

(a)

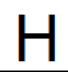

(b)
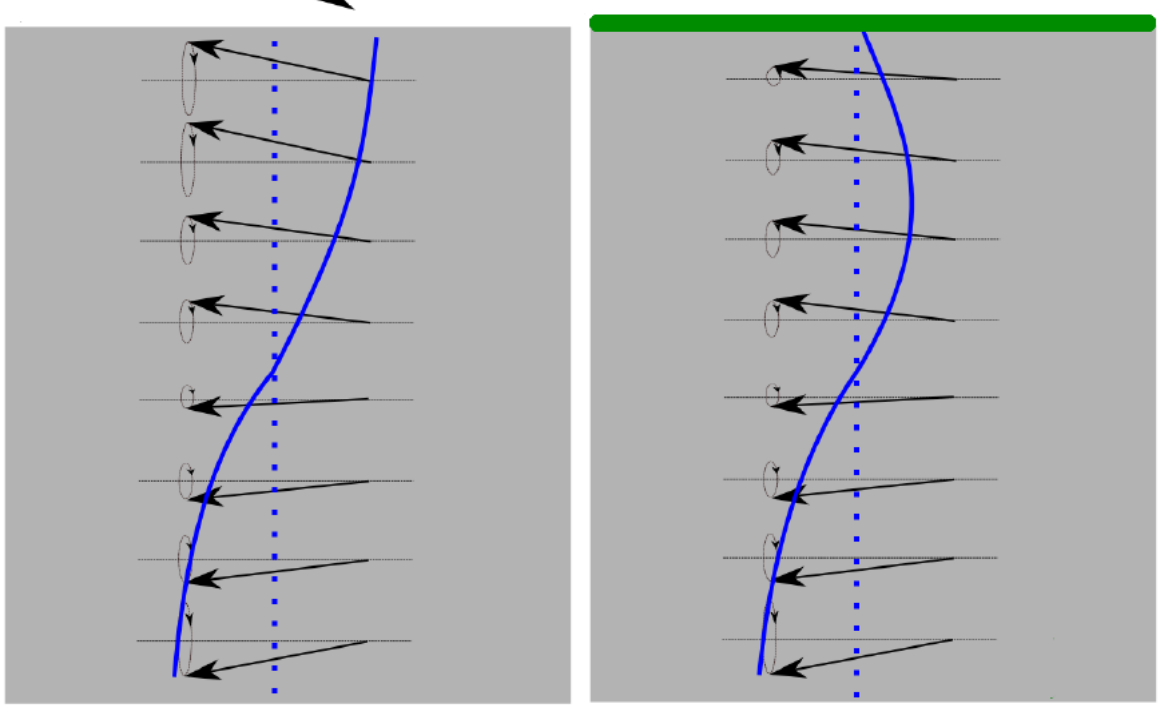

FIG. 3. (a) The unpinned first SSWM profile across the film thickness direction. Arrows in the film (grey area) show an instant snapshot of spin precession. The blue line shows the profile of precession amplitude across the film thickness. Label $\mathbf{H}$ denotes the direction of the applied static magnetic field. One notices that the mean value of the amplitude (the net dynamic magnetic moment) vanishes for this mode for symmetry reason. (b) Spin precession in the presence of spin (magnetisation) pinning at one of the film surfaces (shown schematically by the presence of a thin pinning layer at the top surface of the film). One sees that the precession amplitude is altered at this interface. As a result, the SSWM develops a non-vanishing net dynamic magnetic moment, which may couple to an external magnetic microwave field.

The efficiency of excitation of SSWMs depends on the uniformity of the driving microwave magnetic field of the FMR setup and the uniformity of the material properties over material's 
volume. For instance, observation of SSWMs in thin films requires either the presence of a surface anisotropy or non-uniformity of magnetic parameters across the film thickness. Typical examples are a multi-layered film lacking inversion symmetry or magnetisation pinning at the surface of a single-layer film. The latter case was actually first considered by Kittel [5, 68, 69].

The case of a continuous single-layer film with a normal uniaxial surface anisotropy [70] is the most instructive example. The anisotropy leads to pinning of dynamic magnetisation at the surface of the film where it is present. In the absence of the anisotropy the dynamic magnetisation $\mathbf{m}$ at the external surfaces of the magnetic film satisfies the Rado-Weertman condition [71]

$$
\frac{\partial \mathbf{m}}{\partial \mathbf{n}}=0
$$

If the anisotropy is present and the film is magnetised in its plane, this boundary condition should be generalised as

$$
\begin{aligned}
& \frac{\partial m_{\perp}}{\partial \mathbf{n}}+d \frac{\mathbf{n}}{|\mathbf{n}|} m_{\perp}=0 \\
& \frac{\partial m_{\|}}{\partial \mathbf{n}}=0,
\end{aligned}
$$

where $\mathbf{n}$ is the vector normal to the surface of the magnetic film, and $m \perp$ and $m_{\|}$are, respectively, the perpendicular (PP) and parallel (IP) components of the dynamic magnetisation vector with respect to the surface of the film. In Eqs. $(7,8)$, the effect of pinning is taken into account by means of the surface pinning parameter $d=-K_{\mathrm{u}} / A$, where $K_{\mathrm{u}}$ represents the uniaxial surface anisotropy constant and $A$ is the exchange constant (not to be confused with the exchange stiffness constant $D$ ).

In a multilayer film, in addition to the pinning, the angle of magnetisation precession obeys specific conditions at the internal interface between two magnetic layers [72, 73, 74]. The corresponding (linearised) boundary conditions read

$$
\begin{gathered}
\frac{\partial \mathbf{m}_{1}}{\partial y}+\frac{A_{12}}{A_{1}} \mathbf{m}_{1}-\frac{A_{12}}{A_{1}} \frac{M_{s 1}}{M_{s 2}} \mathbf{m}_{2}=0 \\
\frac{\partial \mathbf{m}_{2}}{\partial y}-\frac{A_{12}}{A_{2}} \mathbf{m}_{2}+\frac{A_{12}}{A_{2}} \frac{M_{s 2}}{M_{s 1}} \mathbf{m}_{1}=0
\end{gathered}
$$

where $A_{12}$ is a constant describing the exchange coupling between the magnetic layers ' 1 ' and ' 2 ', $\mathbf{m}_{1}$ and $\mathbf{m}_{2}$ are the magnetisation vectors in the magnetic layers '1' and '2', and $M_{\mathrm{s} 1}, M_{\mathrm{s} 2}, A_{1}, A_{2}$ are the saturation magnetisations and the exchange constants of the magnetic layers ' 1 ' and ' 2 ', respectively.

The surface pinning means that the magnetisation at the surface cannot precess as freely as in the bulk. Because sharp spatial variation in the amplitude of precession would result in a strong exchange contribution to the resonance energy, the amplitude of the precession can vary only slowly with the distance from the surface, from its surface value to the bulk one. In this way the surface state of magnetisation "propagates" across the bulk of the film which leads to important consequences for experimental detection of the conditions for the magnetisation vector at the surfaces (and interfaces). The case of integer values $n=0,1,2,3 \ldots$ in Eq. (5) corresponds to the absence of the surface pinning. The perfect symmetry/anti-symmetry of the $n=1,2,3$ modes 
[Fig. 3(a)] makes them unobservable while driven by a spatially uniform microwave magnetic field [68]. In the presence of surface anisotropy the mode profiles are deformed [Fig. 3(b)] and SSWMs become driven in a uniform microwave field. As seen from Fig. 3(b), the $n$-values in this case are not integer numbers [40]. Thus, the SSWM frequencies are affected by the inhomogeneous exchange interaction and carry important information about the value of the exchange constant for the material. They also depend on the surface/interface state of magnetisation, and thus, due to the surface state "propagation" through the bulk, one gets access to valuable information about the behaviour of the surface/interface magnetisation vector.

This effect was predicted by Kittel [68] and confirmed experimentally in [40]. It is noteworthy that the frequencies for the higher order modes are more strongly shifted than those for the lower order modes because of the square spin wave dispersion law Eq. (5). This is the best seen for the case of the PP magnetised film.

$$
f=\gamma\left(H_{\mathrm{res}}+H_{e x}-4 \pi M_{s}\right) .
$$

Also, it is worth noting that different types of surface anisotropy pin different vector components of the dynamic magnetisation. Furthermore, the strength of the pinning strongly depends on the orientation of the vector of the static (equilibrium) magnetisation with respect to the anisotropy axis. For instance, if the static magnetisation vector lies in the film plane (i.e. perpendicular to the anisotropy axis) the perpendicular-to-plane component of the dynamic magnetisation can be pinned, but the in-plane component of the magnetisation is free to precess [see Eqs. $(7,8)$ ]. However, if the static magnetisation is aligned along the anisotropy axis, as it happens in the case of a film magnetisation perpendicular to its plane, both components of the dynamic magnetisation (which are now lying in the film plane) can be pinned. Hence, the effect of the surface magnetisation pinning on the SSWM frequencies is generally stronger for the PP film magnetisation than for the IP one.

To understand why different components of the dynamic magnetisation are pinned differently for different directions of the applied field, let us make use of the idea of the effective field of surface anisotropy. In the case of the normal uniaxial anisotropy, the effective anisotropy field is along the film normal. For a film magnetised IP, only the dynamic component of the anisotropy field matters in the linear approximation, since this field is perpendicular to the static magnetisation. This field is $h_{\mathrm{uy}}=2 K_{u} /\left(M_{\mathrm{s}}\right)^{2} m_{\mathrm{y}}[75,76]$. In the same linear approximation, there is no effective anisotropy field $h_{\mathrm{ux}}$ in the direction perpendicular to the direction of the anisotropy axis, i.e. in the film plane. This explains why there is an effect of the uniaxial anisotropy on one vector component of the dynamic magnetisation, but no effect on the other one.

As follows from the formula above, depending on the sign of $K_{u}$ this field either adds to or subtracts from the PP dynamic dipole field $-4 \pi m_{\mathrm{y}}$. The PP dipole field is known to strongly reduce the PP component of the dynamic magnetisation $m_{\mathrm{y}}$ with respect to the in-plane one $m_{\mathrm{x}}$. This may be considered as the effective dipole pinning of $m_{\mathrm{y}}$ all over the film bulk. Similarly, one may expect that the $m_{\mathrm{y}}$ component is further reduced at the surface if easy-plane $\left(K_{u}<0\right)$ surface anisotropy is present. This will be seen as surface pinning of $m_{\mathrm{y}}$. If $K_{u}>0$, this component will be anti-pinned. On the contrary, there will be no direct effect of $h_{\mathrm{ux}}$ on the dynamics of $m_{\mathrm{x}}$, as there is no direct effect of $-4 \pi m_{\mathrm{y}}$ on the dynamics of $m_{\mathrm{x}}$ in the linear approximation. Hence, the boundary condition for this component is "unpinned spins".

When the film is magnetised PP, the effective field of normal uniaxial anisotropy is of different nature. It is now purely static: $H_{\mathrm{uy}}=2 K_{u} / M_{\mathrm{s} \text {. }}$ [75]. $H_{\mathrm{uy}}$ adds to the static demagnetising field 
$-4 \pi M_{\mathrm{s}}$. Similarly to the effect of $-4 \pi M_{\mathrm{s}}$ in this geometry, the effective surface anisotropy field affects both dynamic components of the magnetisation equally. This explains why one obtains the same boundary conditions for both dynamic components of $\mathbf{m}$ in this case. One sees that the applied field orientation with respect to the anisotropy axis rather than to the film plane is important. Every time when the field is applied perpendicular to the anisotropy axis, the dynamic component of the magnetisation along the axis will be pinned and the other one will remain fully unpinned at the surface. Similarly, every time when the applied field is along the anisotropy axis, both dynamic magnetisation components will be pinned, just for the sheer reason of the uniaxial symmetry introduced by the uniaxial anisotropy. For instance, if an IP exchange-bias field is present at one of the film surfaces and the static field is applied in the direction of the exchange bias, both components of $\mathbf{m}$ will be pinned/anti-pinned, although the geometry is one of the IP FMR [77].

As follows from above, the pinning constant scales as the uniaxial anisotropy constant $K_{\mathrm{u}}$. For the easy-axis anisotropy $\left(K_{\mathrm{u}}>0\right)$ the pinning constant is negative. This situation can be termed "anti-pinning" since the dynamic magnetisation precesses more freely at the surface where the anisotropy is present than in the bulk of the film. This results in a surface character of the fundamental FMR mode [10]. Examples of the thickness profiles of the fundamental mode for the cases of $K_{\mathrm{u}}<0$ (easy-plane anisotropy, surface magnetisation pinning) and $K_{\mathrm{u}}>0$ (easy plane anisotropy, anti-pinning) are shown in Fig. 4.

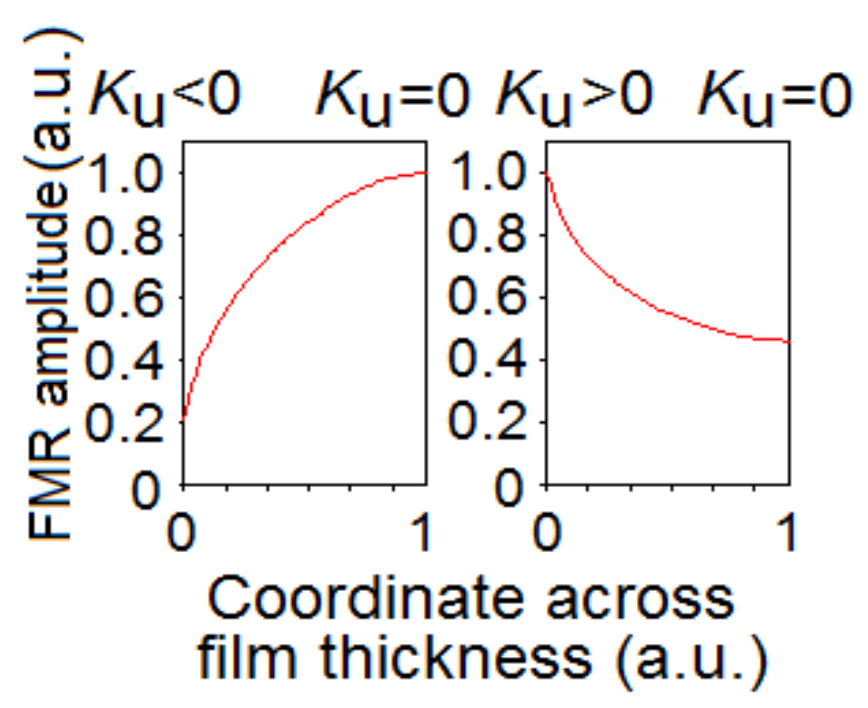

FIG. 4. Thickness profiles of the fundamental FMR mode for the surface magnetisation pinning (left-hand panel) and anti-pinning (right-hand panel). " 0 " and " 1 " of the horizontal axes correspond to the two film surfaces. The surface pinning is present at the left-hand surface of the film only. The spins at the other film surface are unpinned $\left(K_{\mathrm{u}}=0\right)$.

Another useful example is the interface Dzyaloshinskii-Moriya interaction [78, 79, 80, 81]. It pins circular components of the dynamic magnetisation vector. The pinning constants for the leftand right-polarised components are of different signs and scale as the spin- wave wave number [82].

As discussed above, the term "ferromagnetic resonance" or FMR is also employed as the name of a spectroscopy technique with which to probe the magnetisation dynamics in magnetic 
materials. Although, to the best of our knowledge, it is unknown who was the first to employ the FMR spectroscopy, its possible date of birth is somewhere in the 1930s. Early FMR experiments used a microwave cavity to drive magnetisation precession. This configuration is very sensitive since it exploits "resonance amplification" of a usually very weak FMR signal by a microwave cavity. Importantly, the microwave cavity produces a microwave field which is highly uniform on the length scale of a typical magnetic film sample [39, 58, 59, 61, 63, 64].

Modern BFMR setups use a section of a stripline (coplanar or microstrip line) instead of a cavity to drive magnetisation precession. BFMR creates highly inhomogeneous driving rf-fields if the magnetic sample is a good electric conductor (see Section 5). Furthermore, in contrast to the single-frequency cavity FMR, BFMR is broadband (hence the name) and it allows the excitation frequency to be selected, so that resonance conditions may be more fully explored and exploited by, e.g., extracting the Gilbert damping parameter from a series of field-resolved FMR traces taken in a broad range of frequencies. However, because no resonant signal amplification is available with the broadband stripline elements, BFMR is usually less sensitive than the cavity FMR. Consequently, most often one needs to conduct several measurements as the same frequency/applied field and take the average to improve the signal-to-noise ratio.

\subsection{Cavity FMR vs. stripline FMR}

Historically, the first FMR experiments used a microwave (rectangular or cylindrical) resonant cavity with an electromagnet (Fig. 5). A typical cavity FMR setup also consists of a source of microwave power (e.g. a klystron), a microwave waveguide, and a detector diode (e.g. a Schottky barrier diode). The waveguide is used to supply microwave power to the cavity with the sample. The same waveguide is also used to return the signal reflected from the cavity to the detector. A circulator is employed in this scheme to direct the reflected signal to the diode and simultaneously protect the generator from the reflected power.

Given the single frequency operation of the cavity, the measurements are conducted as follows. The generator frequency is tuned to the resonance frequency of the cavity. The cavity with the magnetic sample is placed between the poles of an electromagnet and the magnetic field is swept while the resonant absorption intensity of microwaves is detected. The variation in the applied field "sweeps" the FMR eigen-frequency for the sample. When the FMR frequency and the resonant cavity frequency become equal, the microwave absorption by the cavity increases sharply which is indicated by a decrease in the signal intensity at the detector.

For the measurements the $\mathrm{TE}_{102}$ resonant mode of the cavity [Fig. 5(a)] is exploited, since it ensures high spatial uniformity of the microwave magnetic field right in the middle of the cavity. The microwave field uniformity is very important for the interpretation of experimental results. In particular, uniformity of the microwave field at the position of the sample ensures the absence of microwave eddy currents in the ferromagnetic samples which typically have sub-skin-depth thicknesses (see the discussion in Section 5). Thus, highly conducting samples respond as insulating

films in the cavity FMR method. This considerably simplifies the theoretical interpretation of experimental results.

The resonant cavity has a high quality factor $Q=f_{\text {res }} / \Delta f$, where $f_{\text {res }}$ is the resonant mode frequency of the cavity and $\Delta f$ is the full width at half maximum of the resonance peak. The high quality factor of the cavity explains a very high sensitivity of the cavity FMR. Naturally, $Q$ 
decreases when the magnetic sample is inserted into the cavity. This is because the sample and its holder absorb microwave energy, which in turn slightly shifts the resonance frequency and broadens the resonance peak. These deviations can be compensated by adjusting the iris that controls the effective impedance by varying the size of the aperture between the cavity and the waveguide, or/and by adjusting the position of the metallic cap.

(a)

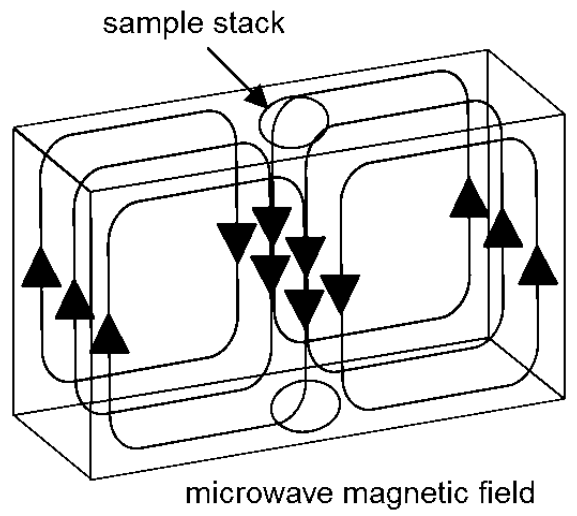

(b)

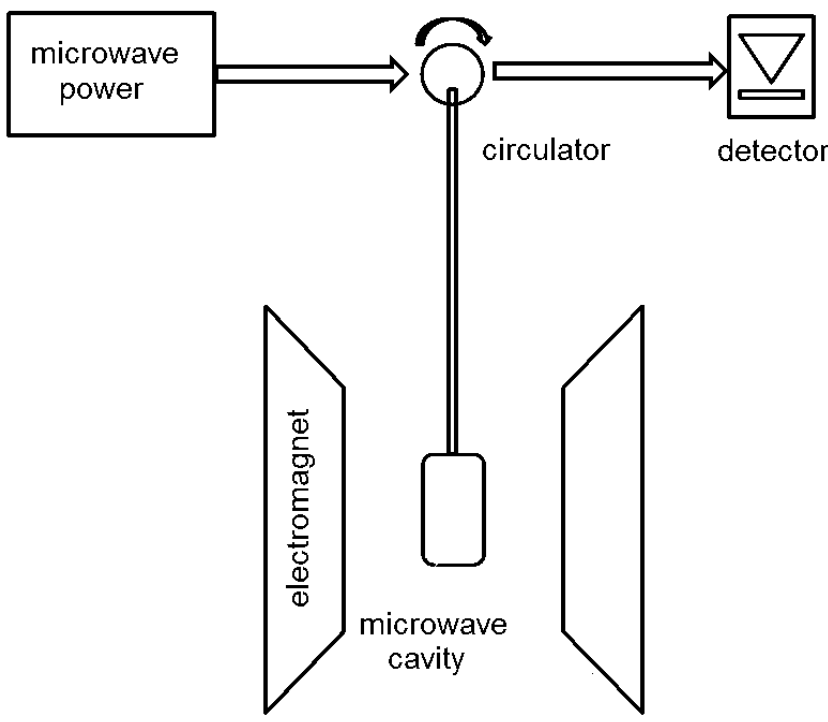

FIG. 5 (a) Microwave magnetic field of the $\mathrm{TE}_{102}$ mode of a rectangular cavity. (b) Basic configuration of the cavity FMR setup. For a relevant discussion see, e.g., Ref. [83].

Apart from very high sensitivity, the other advantages of the cavity FMR are that this technique is well-established and uses standard microwave components. We have already mentioned that the results of measurements in the cavity are easy to interpret due to the microwave field uniformity and the absence of the microwave electric currents in the sample. Similarly, one finds that the higher-order SSWMs do not absorb microwave power and thus are not seen in the cavity FMR spectrum unless the inversion symmetry of the material is broken on the length scale of the free propagation path for travelling spin waves. This conclusion follows from the simple idea that the FMR response driven by a spatially uniform microwave magnetic field scales as the net dynamic magnetic moment (i.e., the mean value of the dynamic magnetisation over the sample volume). A break in the symmetry may occur due to the surface magnetisation pinning in thin continuous films or due to nano-patterning of the films in the film plane (see the discussion in Section 2.1) 
Some of the advantages of the cavity FMR are also its limitations. Because the cavity has a very high $Q$-factor and operates at a single frequency, measurements are conducted for one frequency only and thus the amount of extracted information is very limited. Furthermore, the cavity method is difficult to employ for the characterisations of the samples with very small magnetic losses. When the intrinsic Q-factor of a sample is very high (due to the small losses), coupling of the sample resonance to the cavity resonance may be very strong which may lead to the shift the resonance frequency of the cavity $[84,85]$ and thus make the measurement of the FMR frequency impossible.

Furthermore, the resonant frequency of a cavity may be too low in order to observe some magnetisation precession modes. For instance, at $9.5 \mathrm{GHz}$, which is the typical frequency of operation of commercially available Electron Spin Resonance spectrometers, no FMR response will be seen for many nano-patterned materials (see, e.g., Fig. 3 in [86]). One may also expect that no SSWMs will be seen in the spectra of very thin ferromagnetic films at $9.5 \mathrm{GHz}$.

The disadvantages of the cavity FMR are not present in the stripline BFMR method. The stripline transmission line is a type of a microwave waveguide. In the following, we will be interested in the microstrip [Fig. 6(a)] and coplanar [Fig. 6(b)] microwave transmission lines. We will use the notion of a "stripline" as a more general term which refers to both coplanar and microstrip lines. On the contrary, when particular stripline types are important for particular contexts we will employ the terms "microstrip line" and "coplanar line".

The planar structure of striplines and easy accessibility of the area of localisation of their microwave field make them very convenient for the characterisation of thin films and planar nanostructures. Furthermore, being naturally broadband microwave waveguides, the striplines can be employed for taking measurements in a broad frequency range, often from several hundreds of $\mathrm{MHz}$ to 30-40 GHz [41, 42]. The magnetic sample is mounted on top of the stripline [Fig. 6(c)]. The magnetisation dynamics in the sample is induced by a microwave Oersted (dynamic) magnetic field, which is created by a microwave current flowing through the signal line of the stripline. 

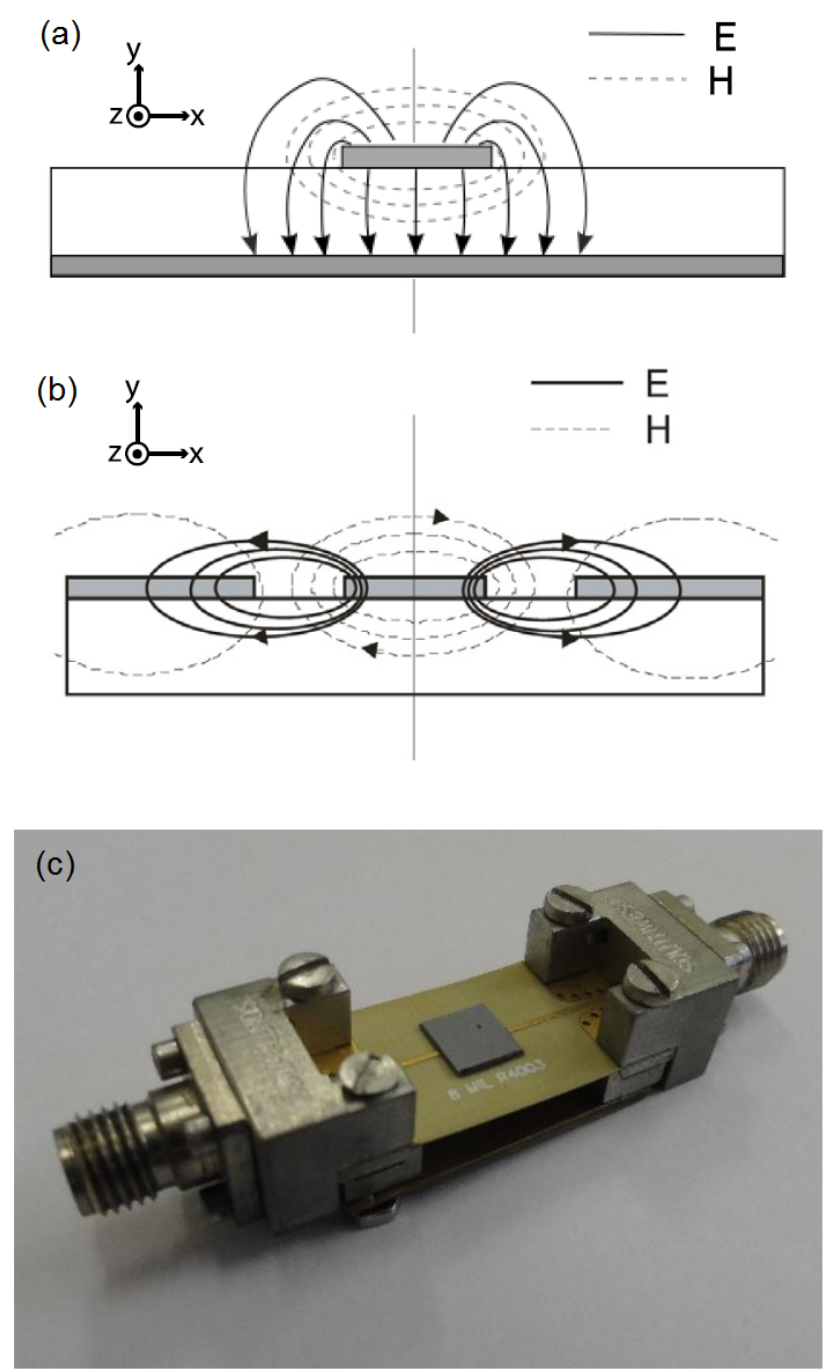

FIG. 6. Schematic of the microwave magnetic field of an unloaded microstrip line (a) and coplanar line (b). After [87]. The microwave electric field distribution is shown by the solid lines and the distribution of the microwave magnetic field by the dashed lines. The thin vertical lines in (a) and (b) show the symmetry axes of the geometries of the microstrip and coplanar lines. (c) Photograph of a microwave microstrip line with the sample under test (dark grey square) across the signal line. This fixture was used to take the measurements in [88].

A microstrip line represents combination of a metal strip of a finite width $w$ called the signal line (or sometimes "microstrip") and a parallel ground plane of infinite width. The two metallic planes are separated by a layer called the substrate made from a microwave dielectric material. A coplanar line represents a combination of three parallel planar metallic electrodes, all lying in the same plane. The central electrode has a finite width $w$ and is called the signal line. The two other electrodes represent half-planes and are called the ground lines. The electrodes are separated by two air gaps.

Numerically simulated distribution of the microwave electric potential over the crosssection of a microstrip line is shown in Fig. 7(a). An important observation from Fig. 6(a) is that the microwave magnetic field of a microstrip has two components: an in-plane (IP) one and an perpendicular-to-plane (PP, also called out-of-plane) one. They are characterised by similar peak amplitudes but different spatial symmetries: the IP one is symmetric with respect to the vertical 
symmetry axis of the microstrip line [shown by the thin vertical lines in Fig. 6(a) and (b)], but the PP one is anti-symmetric (and hence vanishes on the symmetry axis, see Fig.(7b)). The similarity of the peak amplitudes for the two field components is a fundamental property of an Oersted field of a plane sheet of a current: it follows from the circulation law for the Oersted field (see Eq. (15) in Ref. [89]). The microwave magnetic field of a coplanar line is more complicated [Fig. 6(b)]. The IP component is mostly localised above the signal (central) line (also called "track") of the coplanar line and the PP component in the slits separating the signal line from the ground lines (half-planes).

In a stripline FMR experiment, one measures microwave absorption as a function of the driving microwave frequency or externally applied static magnetic field while keeping the applied field or frequency (respectively) fixed. At resonance, a dip in the trace of microwave power transmitted through the stripline vs. frequency or applied field indicates absorption of microwave power into the sample. The experiment is usually repeated for a number of frequencies or static magnetic fields (respectively). This feature makes the stripline FMR very useful for studying different material parameters such as the damping constant $\alpha_{\mathrm{G}}$ and $g$-factor (see, e.g., [41, 42]). These parameters are extracted from experimental data by fitting them with the appropriate analytical formula [e.g., the Kittel equations Eq. (2)] or numerical simulation (see, e.g., Ref. [90]).

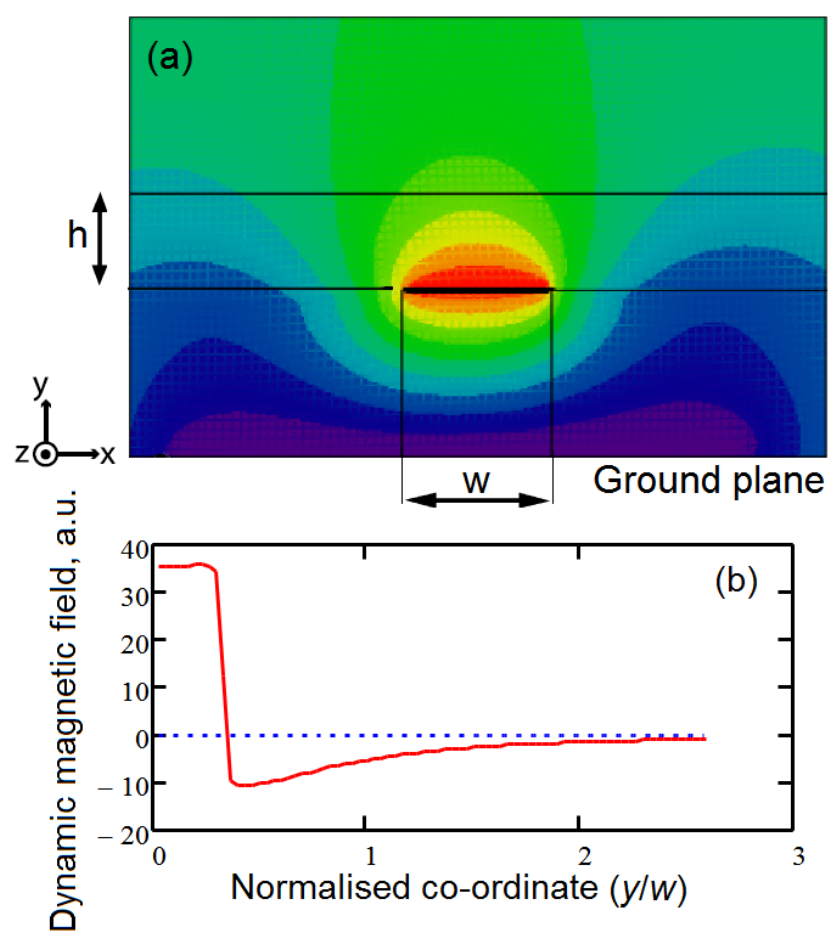

FIG. 7. (a) Simulated distribution of the electric potential over a $(x-y)$ cross-section of a microstrip line loaded with a thin film sample. The width of the microstrip line is $w=1.5 \mathrm{~mm}$. The parameter $h$ denotes the thickness of the substrate of the sample. (b) The in-plane (solid line) and perpendicular-to-plane (dotted line) component of the microwave magnetic field of a microstrip line as a function of the $y$-coordinate. This profile is along the vertical symmetry axis of the geometry in Fig. 6(a). In this graph the microstrip ground plane is at 0 and the microstrip is located at 0.3 (the distance is normalised to the microstrip width $w$ ). This profile was calculated with customised software developed by one of the authors (M.K.). The numerical method is based on the duality of the microwave electric and magnetic fields and the approach from [91] to calculate the microwave electric field of the microstrip line. 


\subsection{Pulse Inductive Microwave Magnetometry}

Historically, most of the early stripline FMR spectrometers [92] mimicked the "Fourier Transform Method" of operation of Nuclear Magnetic Resonance spectrometers [93] - they exploited broadband pulse excitation of the stripline. The method got the name "Pulsed Inductive Microwave Magnetometry" or shortly PIMM $[92,94]$. Below we describe it on the example of the setup at the University of Western Australia ${ }^{1}$.

The magnetic sample is placed upon a coplanar line (Fig. 8). The sample is electrically isolated from the waveguide by a thin separating layer of teflon. The sample can then be placed face-side down to maximise the inductive coupling with the field to the coplanar line. The coplanar line is connected to the measurement system via coaxial cables.

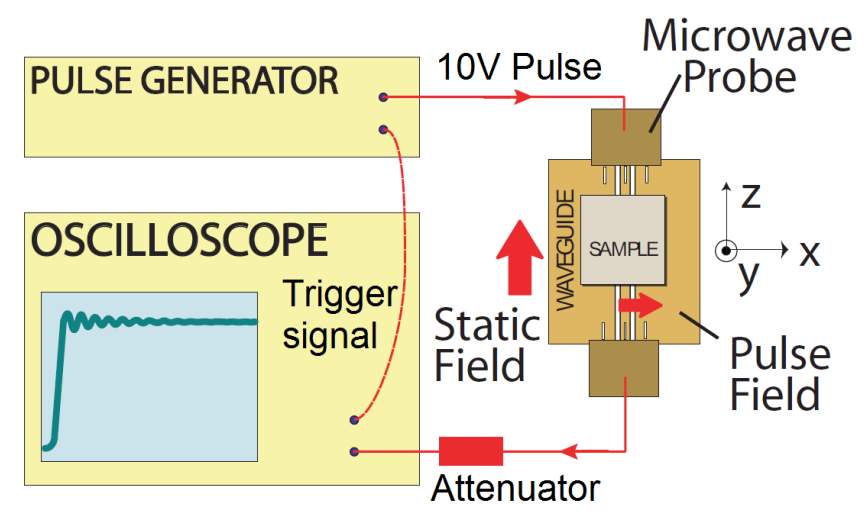

FIG. 8. Schematic of the PIMM setup described in the main text. The excitation field is created by a pulse generator, and the transmitted pulse is measured with a sampling oscilloscope.

An excitation field is launched onto the waveguide from a step pulse generator. The pulses have a nominal rise time of $50 \mathrm{ps}$, a pulse duration of $10 \mathrm{~ns}$, and are outputted with a repetition rate of $10 \mathrm{kHz}$. The excitation field disturbs the equilibrium position of the magnetisation by altering the effective field direction. The magnetisation precesses around this new equilibrium position. The precessing magnetisation induces a voltage back into the coplanar line.

The harmonic part of the signal is exponentially damped due to energy dissipation within the sample, and is super-imposed onto the original much larger $10 \mathrm{~V}$ excitation pulse. This is measured by a $20 \mathrm{GHz}$ sampling oscilloscope, measuring 512 pulses. As the period between the pulses $(0.1 \mathrm{~ms})$ is much larger than the relaxation time of the magnetisation, the measurement is completely repeatable. Noise is reduced by averaging the measurement a few hundred times. Time Fourier transformation of measured data is used to obtain the susceptibility of the investigated magnetic film. The susceptibility for a Permalloy $\left(\mathrm{Ni}_{80} \mathrm{Fe}_{20}\right)$ thin film is shown in Fig. 9.

This setup was developed by K. J. Kennewell, R. Woodward and R. L. Stamps under guidance by T. Silva from the National Institute of Standards and Technology (NIST) Boulder, Colorado, USA. This description is taken from the PhD thesis by K. J. Kennewell. 


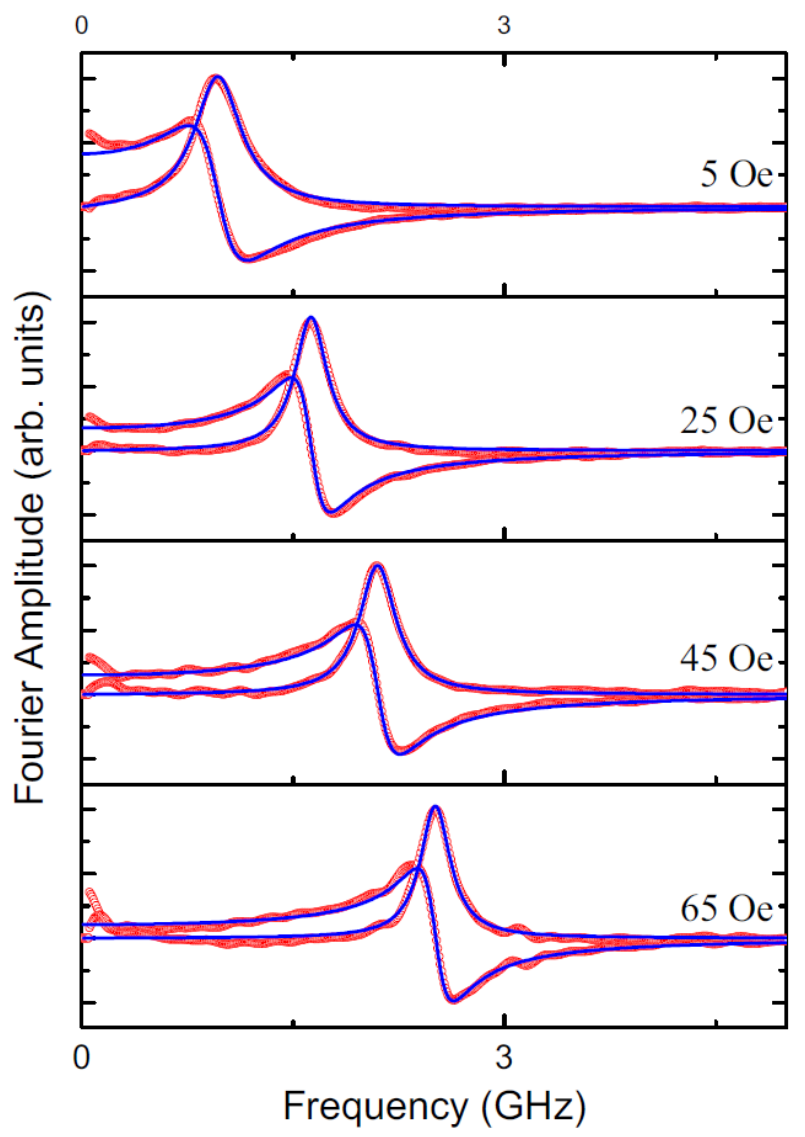

FIG. 9 Susceptibility spectra of a Permalloy $\left(\mathrm{Ni}_{80} \mathrm{Fe}_{20}\right)$ thin film for different applied fields calculated from the Fourier transformed PIMM data. Both the real and imaginary parts are shown (red open circles). The real part of the data exhibits a crossing and the imaginary part of the data exhibits a peak at the samples resonant frequency. The solid blue lines are, respectively, the best fits to the Lorentzian (bell) shape characteristic and a sign varying function featuring a characteristic $\pi$ phase jump at the resonance frequency. (The red open circles largely overlap making an impression of a thick red solid line.)

The results in Fig. 9 are seen to be dependent on the applied bias field $H$, with the frequency increasing with field. In this case the Kittel equation reads

$$
f^{2}=\gamma^{2}\left(H_{\mathrm{K}}+H\right)\left(H_{\mathrm{K}}+H+4 \pi M_{s}\right),
$$

where $H_{\mathrm{K}}$ is the effective magnetic field of magnetocrystalline anisotropy. By plotting the square of the resonant frequency versus $H$ and fitting the resulting curve to the Kittel equation, one can extract the values of $H_{\mathrm{K}}$ and $g$ from the gradient by taking the approximation when $H_{\mathrm{K}}+H<<4 \pi M_{\mathrm{s}}$. Moreover, the parameter $\Delta f f f$, which is a measure of the resonance peak line width, can be extracted from the Fourier transformed data and used to extract the Gilbert damping constant $\alpha_{\mathrm{G}}$.

To summarise the discussion of the PIMM, we notice a considerable complexity of the postprocessing of the experimental data due to the time-domain origin of this spectroscopy technique. Whereas the PIMM benefits from broadband properties of the stripline, the post-processing and analysis of the data require complex Fourier transformations. Furthermore, each step of 
mathematical processing of data registered with a finite experimental accuracy "amplifies" the uncertainty in the values of material parameters extracted from the experiment. This makes the PIMM a much more complex and less accurate method as compared with the cavity FMR. Furthermore, the costs of the nanosecond pulse generator and the broadband oscilloscope are typically higher than the costs of a microwave generator and a detector used in the cavity FMR. Some of the disadvantages of the PIMM - its time-domain character and the complex postprocessing - are not present in the VNA FMR configuration, which is discussed in the next section.

\subsection{Vector Network Analyser (VNA) FMR}

The Vector Network Analyser (VNA) FMR is used to study excitations of the magnetisation dynamics in thin magnetic films and high-quality magnetic nanostructures (see, e.g., [46, 88, 92, 95, 96, 97, 98, 99, 100, 101, 102, 103, 104, 105, 106, 107, 108, 109, 110]). These nanostructures have attracted a lot of attention due to the advent of modern fabrication technologies allowing one to fabricate high-quality thin magnetic films as well as single and periodic nanostructures. The latter are often called "magnonic crystals" [14, 15, 16, 111].

By analogy with the PIMM, the VNA FMR is an inductive method to study the magnetisation dynamics in magnetic films and nanostructures because the magnetisation dynamics in the sample under test is induced by a microwave field. This field can be created, for example, by a microwave current flowing through the signal line of a microwave stripline (Fig. 10).

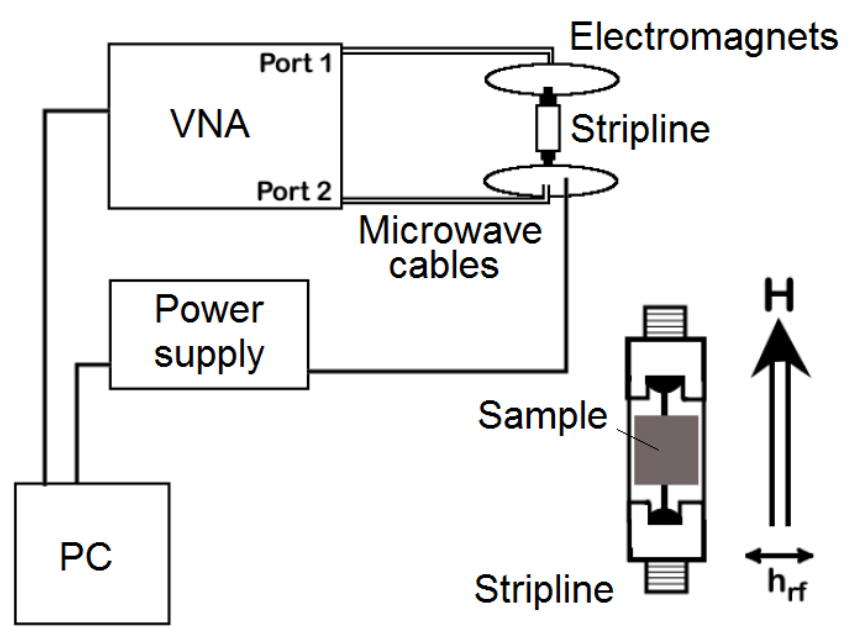

FIG. 10. (a) VNA-FMR setup displayed schematically, with the stripline and sample geometry in the bottom right. Shown is the applied field direction $H$, relative to the rf microwave field $h_{\mathrm{rf}}$. VNA denotes the Vector Network Analyser, PC denotes the Personal Computer.

The stripline with the sample under test on top of it is placed between the poles of an electromagnet (Fig. 10) such that the line is parallel to the direction of the applied static magnetic field (so-called in-plane FMR configuration). The stripline is connected on both ends to the two ports of a VNA. The VNA functions as both a source of a microwave signal to excite the magnetisation dynamics in the magnetic sample, and as a signal receiver. More precisely, it 
measures the scattering parameters $-S_{21}$ (transmission) and $S_{11}$ (reflection) - of the device under test (DUT). The values of the scattering parameters represent proxies to the strength of the microwave magnetic absorption by the sample. One measures the microwave absorption as a function of the driving microwave frequency and/or externally applied static magnetic field. Resonant absorption of microwave power by the sample is seen as a dip in the measured spectrum. There are two methods to measure the FMR response of the sample: frequency sweep and field sweep. They are discussed below.

In the frequency sweep method, the field of the electromagnet is fixed, and the scattering parameters are measured as a function of frequency. This method is quick, but less sensitive compared to a field sweep. In addition, frequency sweeps may yield signals which are nonmagnetic in origin, but simply due to variations in the impedance of the DUT as the frequency is swept. Therefore, the raw experimental data require post-processing to eliminate the parasitic resonances from the traces. This will be explained below.

An example of the spectrum taken with the VNA FMR using the field sweep method is shown in Fig. 11. In this method, the VNA is set to operate at a single frequency, and the electromagnetic field is swept. The same scattering parameters are measured, but now as a function of the field. This method is slower, because it is usually impossible to vary the applied field with the same rate as VNA may sweep the microwave frequency. However, it is more sensitive than a frequency sweep. In addition to the advantage in sensitivity, it only yields signals which vary with the magnetic field. Ensuring that all microwave parts of the setup which are in contact with the magnetic field (such as cables, coaxial-to-stripline adapters, and the stripline itself) do not contain magnetic materials, removes the parasitic magnetic background completely. This gives the fieldsweep method an important advantage with respect to the frequency sweeps.

Here one has to note that this method fails if the measurements are to be taken on magnetically unsaturated samples, because varying the applied magnetic field will gradually change the ground state of magnetisation. Therefore, for taking measurements inside hysteresis loops for a material the frequency swept FMR is more appropriate [54, 60, 112, 113, 114, 115].

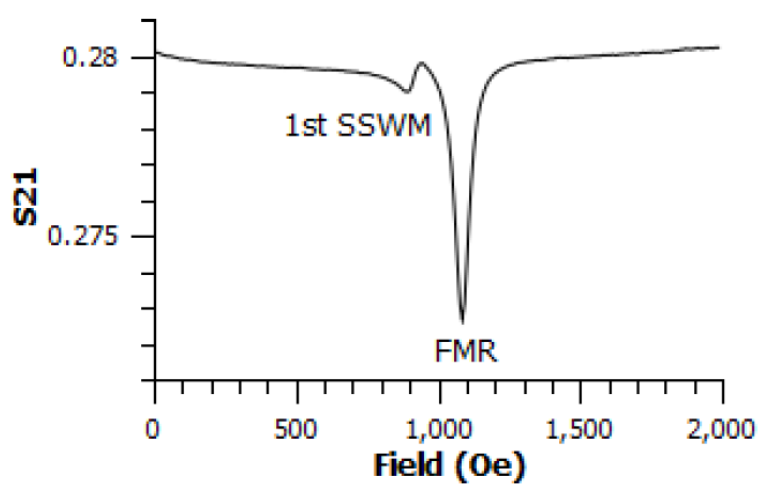

FIG. 11. Microwave absorption spectrum of a $100 \mathrm{~nm}$ thick Permalloy film at the frequency of $10 \mathrm{GHz}$, showing the fundamental mode (labelled as "FMR" in the figure) and the $1^{\text {st }} \mathrm{SSWM}$ as absorption dips.

In all cases of the VNA FMR, the measured quantities are $S$-parameters. Similar to the 
PIMM, the relationship of the $S$-parameters to the resonance in the sample (via susceptibility of the sample) should be determined. The $S$-parameters represent ratios of the complex pre- and posttransmission (and reflection) standing power waves as discussed in detail in [116].

To summarise Section 2.4, we have discussed the advantages and disadvantages of the VNA FMR. The merit of the VNA FMR is that it enables one to take measurements of the value of the microwave magnetic absorption in terms of well-defined scattering parameters and in absolute units. The complex S-parameters are measured with a standard commercially available tool (a VNA) that also serves as the source of microwave power. Usually, a VNA is supplied with embedded software and calibration kits allowing one to quickly calibrate the device.

The disadvantage of this technique is that it measures the scattering parameters of the whole DUT - both the stripline line and the sample of interest. Due to a significant difference between the stripline characteristic sizes and the sample thickness, the magnetic part of the absorption signal inserted by the sample is almost always much smaller that the total signal from the output of the stripline, appearing as a blip on top of the background stripline signal. Typically, background subtraction needs to be done to isolate the sample signal from the total DUT signal. It complicates post-processing and interpretation of the data.

An important exception is the samples made from single-crystal yttrium-iron garnet (YIG). Due to exceptionally small magnetic losses for this material the magnetic absorption by those samples is most often comparable to the non-magnetic background. Thus, no background subtraction is needed in this case.

\subsection{Field-modulation FMR (FM FMR) and interferometric detection of weak FMR signals}

Due to the aforementioned disadvantages of the VNA FMR, lock-in and modulation BFMR techniques are also often used [64, 61, 117, 118]. In these cases, the VNA is replaced by a microwave generator, a microwave diode, and a lock-in amplifier. In addition, modulation coils are fixed at the poles of the electromagnet (Fig. 12). Given high costs of a brand-new VNA, this method is much cheaper to implement which may be an important factor in many cases; especially if some of the above listed equipment pieces are already available in the lab.

The microwave signal transmitted through the DUT is measured as a function of applied field for given microwave frequencies. Alternatively, the reflected signal can also be measured by redirecting the power reflected from the DUT to the diode with a circulator. Similar to [99, 103], the field is modulated using two small coils attached to the poles of the electromagnet. The setup used at the University of Western Australia employs the modulation frequency of $220 \mathrm{~Hz}$, and the AC magnetic field produced by the coils is typically between 1 and 9 Oe, depending on the anticipated linewidth of the measured resonances. This input power is set such that the rectified bias voltage at the output end of the tunnel diode is in the most sensitive and linear region of the particular diode's response. The transmitted/reflected signal from the DUT is rectified using the diode and fed into a lock-in amplifier referenced by the same $220 \mathrm{~Hz}$ signal driving the modulation coils. The signal obtained in this way is proportional to the field derivative of the imaginary part of the microwave susceptibility $\chi[103]$.

Consider the microwave susceptibility of the DUT $\chi(H)$ as a function of the magnetic field $H$. The modulation produces an AC field on top of the DC field, so the susceptibility becomes 
$\chi\left(H+h e^{i \omega t}\right)$, where $h$ is the magnetic field of the modulation coils and $\omega$ is the modulation frequency. The first two terms of the Taylor expansion (with respect to time) of the susceptibility are

$$
\chi(H)+i \omega h \mathrm{e}^{i \omega t} \frac{\mathrm{d} \chi}{\mathrm{d} H} .
$$

This signal is fed into the lock-in amplifier. The first term is effectively a DC term, which gets filtered out. The second term is an oscillatory signal with the same frequency as the field modulation frequency. By referencing the lock-in amplifier with the driving frequency of the modulation coils, the second term gets "locked-in".

It is worth noting that the second term is proportional to the modulation amplitude and the shape of the curve is the first derivative of the susceptibility curve ("differential absorption"). This curve can be fit with analytical and numerical model in order to extract important material constants such as the Gilbert damping parameter. However, in contrast to the VNA FMR, the direct extraction of the amplitude of the FMR and higher-order SSWMs in absolute units is hardly possible, or at least would require a sophisticated analysis of the microwave track between the generator and the diode (which is automatically done by the VNA built-in software in the case of the VNA FMR). Note that in order to extract the full width at half maximum (FWHM) of the FMR resonance from the first derivative of the susceptibility curve, one needs to measure the distance between the two peaks (the positive one and the negative one) of the first derivative of the susceptibility curve and multiply this distance by $\sqrt{3}$.

For those who are accustomed to analysing the VNA FMR traces, a differential absorption trace may look unusual. However, by taking a first "anti-derivative" of a raw differential absorption trace numerically one easily retrieves the conventional Lorentzian shape for the resonance lines typical for the raw VNA data (Fig. 13). Here one has to note that often the features in differential absorption traces, such as a fine peak structure, are more pronounced as in the "anti-derivative" (i.e. in the equivalent VNA data), as seen in Fig. 13. This is due to the simple fact that differentiating emphasises any changes in the slope present in a curve. 


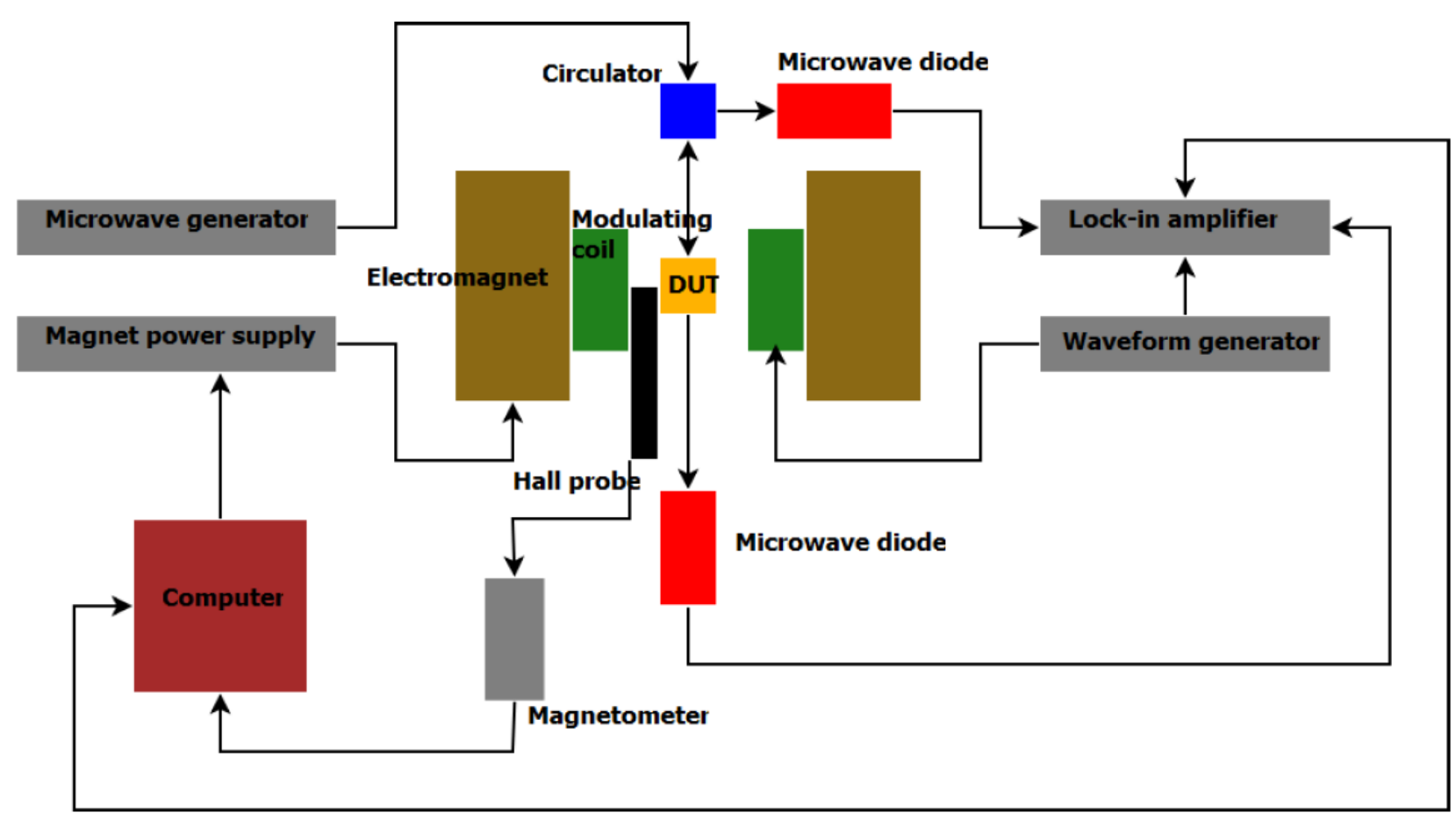

FIG. 12 Lock-in with field modulation broadband method circuitry.

Furthermore, typically, background signals from the sample itself, the stripline and other potentially magnetic components located between the electromagnet poles are broadband features. However, signals from the magnetic resonances in the sample are typically sharp. Consequently, the derivative of the background signal is effectively vanishing in the differential absorption data as compared to the sample resonance signal. The practical absence of background means that the sensitivity of the lock-in amplifier can be set to the sample signal level. Furthermore, one does not need to take a background sweep as necessary in the case of the frequency-swept VNA FMR.

It is noteworthy that $1 / f$ noise [119] in the collected data can be reduced by increasing the modulation frequency. Furthermore, as follows from Eq.(13), the amplitude of the FM response and hence the setup sensitivity scale as the modulation frequency. Thus, increasing the modulation frequency is beneficial. Here one has to take care that the modulating coils are located well away from the pole pieces of the electromagnet and as close as possible to the sample. If they are attached to the poles of the electromagnet (as in Fig. 12), due to the large contribution from the magnet yoke, the coil inductance will quickly increase with modulation frequency resulting in a quick drop in the current through the modulating coil and hence in the AC field it produces for a given AC voltage supplied by a function generator. For a coil sitting on a magnet pole piece frequencies of modulation of a couple of hundreds of Hertz are typical [120], but with a standard Varian-IV cavity with built-in modulation coils modulation frequencies of several tens of $\mathrm{kHz}$ are easily achievable [121]. 


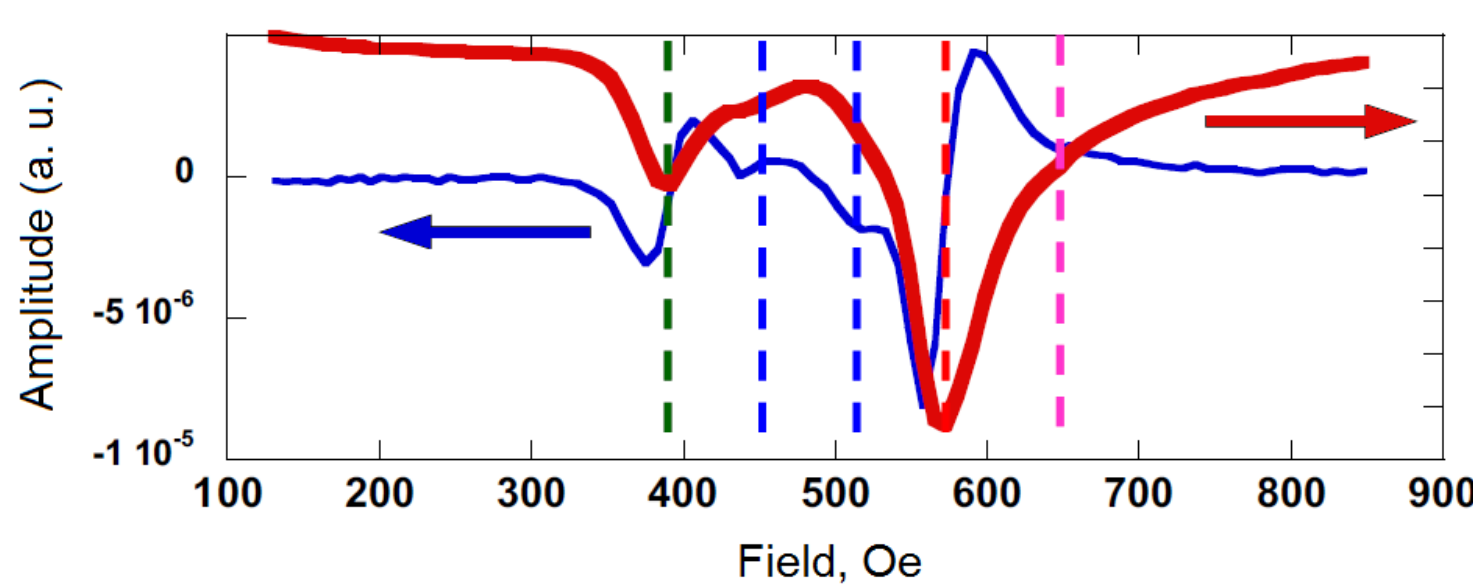

0

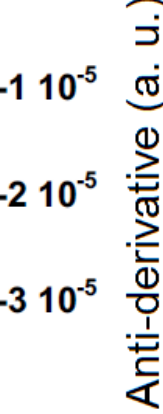

FIG. 13 Differential absorption curve (blue line) and its first anti-derivative (equivalent to a VNA FMR trace) taken on a magnetic nano-dot array. One sees that the fine structure of the resonance line is significantly better visible in the differential data than in the equivalent VNA trace [122].

All the above considered, a single run of the FM FMR yields much better signal-to-noise ratio compared to VNA traces taken without averaging over numerous VNA measurement runs. A further decrease in the noise level in the FM FMR data is achieved by increasing the lock-in time constant and collecting the lock-in signal for a longer time accordingly (at least 5 to 6 time constants). This is equivalent to an increase in the number of VNA runs in the VNA FMR method over which the FMR signal is averaged.

However, the most radical reduction in the noise floor is achieved by replacing the microwave diode with a microwave interferometric instrument ("receiver") [123]. With this custom-build receiver a single measurement run allows one to register FMR signals with amplitude of just $100 \mathrm{nV}$ with no noticeable noise background at all when the lock-in time constant is set to $0.3 \mathrm{~s}$ which is just 60 periods of the field modulation [120], with plenty of room for the further noise level improvement by increasing the field modulation frequency (see the discussion above of the importance of proper positioning of the modulation coils).

The receiver is easy to tune and represents quite a small investment compared with a typical price of a brand-new VNA. Importantly, sensitivity to the signal of either real or imaginary part of the complex microwave magnetic susceptibility can be achieved by proper tuning of the receiver. Moreover, it is even possible to measure both parts simultaneously by investing slightly more into the microwave parts. In the latter case, one eliminates the main disadvantage of the FM FMR method with respect to the VNA FMR one - the impossibility of measuring the real and imaginary parts of the complex FMR response simultaneously.

The authors of this paper now employ the interferometric instrument for most of their FMR measurements.

\subsection{In-plane vs. perpendicular-to-plane FMR}

Magnetic multilayer materials with strong perpendicular magnetic anisotropy are amendable for use in emerging spintronics [33, 124, 125] and next-generation data-storage technologies [126] due to the high degree of tuneability of both anisotropy and saturation magnetisation. Such multi-layers can be formed from alternating two ferromagnetic materials such as $\mathrm{Co} / \mathrm{Ni}$ [127] and $\mathrm{CoFe} / \mathrm{Ni}$ 
[128], or alternating a ferromagnetic material with a non-ferromagnetic material such as $\mathrm{Co} / \mathrm{Pt}$ [129, 130, 131, 132], $\mathrm{Co} / \mathrm{Pd}$ [133], $\mathrm{CoFe} / \mathrm{Pd}$ [134], and $\mathrm{CoNi} / \mathrm{Pd}$ [135]. The high-frequency behaviour of these materials is of considerable technological interest due to the strong dependence of spin-torque critical currents and patterned-media switching rates on the degree of damping for gyromagnetic precession [136]. However, measurements of damping in multi-layers by means of FMR can be challenging in the case of strong perpendicular anisotropies (which require high measurement frequencies in excess of $30 \mathrm{GHz}$ ) and/or broad FMR line widths (which necessitate high instrument signal-to-noise ratio and stability) [41, 137, 138, 139].

Néel was the first to propose that an interface or surface will generate a perpendicular anisotropy due to the broken symmetry of a surface or interface [140]. However, the fundamental origin of this effect has been the focus of much debate over the past two decades. Many investigations on the origin of surface anisotropy have focused on the measurement of the orbital and spin moments of magnetic interfaces and surfaces [141, 142, 143, 144, 145, 146]. Nevertheless, measurement of the orbital moment in materials along specific directions remains an experimental challenge. Whereas synchrotron facilities are required to conduct $\mathrm{X}$-ray magnetic circular dichroism (XMCD) measurements [147, 148], the orbital moment can also be determined in the laboratory using FMR through careful evaluation of the spectroscopic splitting factor $g[59,149]$.

Measurements of the $g$-factor were made accessible due to the increased availability of broadband VNA's discussed in Section 2.4. However, precise $(<1 \%)$ determination of the $g$-factor of thin films has remain a challenge for the in-plane VNA FMR configuration due to the nonlinear dependence of the resonance frequency on the resonance field [see Eq. (5)] resulting in a difficulty of fitting the experimental data with this equation. This drawback is less present in the perpendicular-to-plane FMR configuration, in the first place due to the linear law Eq. (4). This becomes of high importance for precise measurements. Moreover, in the PP geometry the contribution of two-magnon scattering to the measured line width is minimised [150]. Another important advantage of the PP FMR arises specifically for the samples with the normal (perpendicular) surface/interface uniaxial anisotropy. As we have shown above (Section 2.1) in this case both components of the dynamic magnetisation are pinned at the surface/interface possessing the interface anisotropy, whereas in the conditions of the IP FMR only the PP component of dynamic magnetisation feels the presence of the normal surface/interface anisotropy. Thus, one may expect a stronger contribution of the normal uniaxial anisotropy to the PP FMR data.

It is known that the resonance is described by the complex susceptibility $\chi\left(H_{\text {res }}\right)$ derived from the LLG equation [Eq. (1)]. For the perpendicular-to-plane configuration the susceptibility for a single-layer continuous film reads

$$
\chi\left(H_{\text {res }}\right)=\frac{M_{\text {eff }}\left(H_{\text {res }}-M_{\text {eff }}\right)}{\left(H_{\text {res }}-M_{\text {eff }}\right)^{2}-H_{\text {eff }}^{2}-i \Delta H\left(H_{\text {res }}-M_{\text {eff }}\right)},
$$

where $H_{\text {res }}$ is the resonance field, $H_{\text {eff }}=2 \pi f /\left(\gamma \mu_{0}\right)$ is the effective field, $M_{\text {eff }}$ is the effective magnetisation, $\Delta H$ is the line width, and also $f$ is the frequency and $\gamma=\left(g \mu_{\mathrm{B}}\right) / \hbar$ is the gyromagnetic ratio being $\hbar$ the reduced Plank's constant. The VNA FMR technique is sensitive to both the amplitude and the phase, and therefore both the real and imaginary parts of the susceptibility can be measured. It can be done by simultaneously fitting the real and imaginary spectra to Eq. (14) in order to determine $H_{\text {res }}$ and $\Delta H$ [138]. According to the definition of the anisotropy energy density $E$ given in Ref. [151], which includes the second-order $\left(K_{2}\right)$ and fourth-order $\left(K_{4}\right)$ perpendicular 
anisotropies,

$$
E=\frac{\mu_{0} M_{s}^{2}}{2} \cos ^{2} \theta-K_{2} \cos ^{2} \theta-\frac{K_{4}}{2} \cos ^{4} \theta
$$

where $\theta$ is the polar angle relative to the sample normal (perpendicular) direction. The Kittel equations for the perpendicular-to-plane $(\perp)$ and in-plane $(\|)$ geometries in a saturated state are given by Eqs. $(16,17)$

$$
\begin{array}{r}
f\left(H_{\mathrm{res}}\right)=\frac{g^{\perp} \mu_{0} \mu_{B}}{2 \pi \hbar}\left(H_{\mathrm{res}}-M_{\text {eff }}^{\perp}\right), \\
f\left(H_{\mathrm{res}}\right)=\frac{g^{\|} \mu_{0} \mu_{B}}{2 \pi \hbar} \sqrt{H_{\mathrm{res}}\left(H_{\mathrm{res}}+M_{\text {eff }}^{\|}\right)} .
\end{array}
$$

Using Eq. (14), the perpendicular-to-plane $\left(M_{\text {eff }}^{\perp}\right)$ and the in-plane $\left(M_{\text {eff }}^{\|}\right)$effective magnetisations become

$$
\begin{gathered}
M_{\text {eff }}^{\perp}=M_{\mathrm{s}}-\frac{2 K}{\mu_{0} M_{\mathrm{s}}}=M_{\mathrm{s}}-\frac{2 K_{2}}{\mu_{0} M_{\mathrm{s}}}-\frac{2 K_{4}}{\mu_{0} M_{\mathrm{s}}}, \\
M_{\text {eff }}^{\|}=M_{\mathrm{s}}-\frac{2 K_{2}}{\mu_{0} M_{\mathrm{s}}},
\end{gathered}
$$

Examples of perpendicular-to-plane and in-plane geometry data with fits to the Kittel equations are given in Fig. 14 [41]. As stated earlier, precise $(<1 \%)$ determination of the $g$-factor is challenging for the in-plane geometry because, as Eq. (17) shows, the relationship between $f$ and $H_{\text {res }}$ is nonlinear unless $H_{\text {res }}>>M_{\text {eff }}$. As a result, measurements must be conducted over a very large range of $f$ and $H_{\text {res }}$ in order to obtain reliable fits. 
(a)

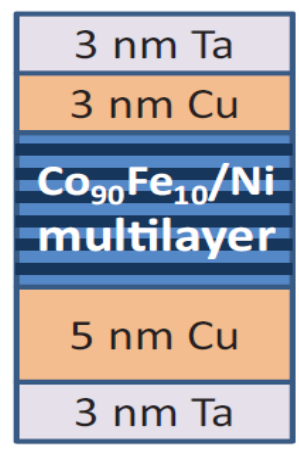

(b)

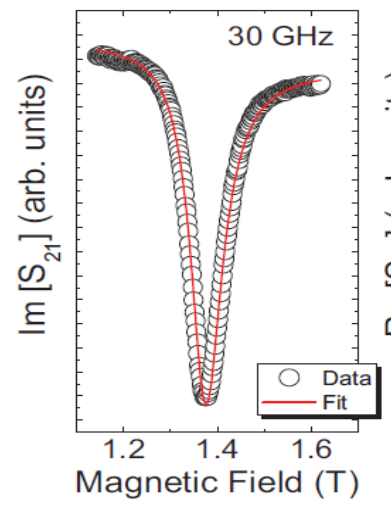

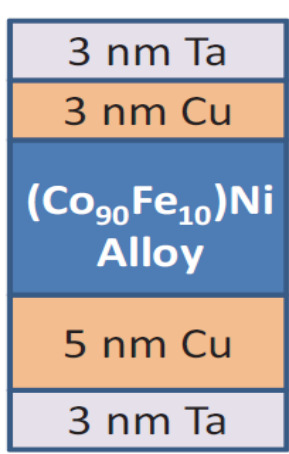

(d)

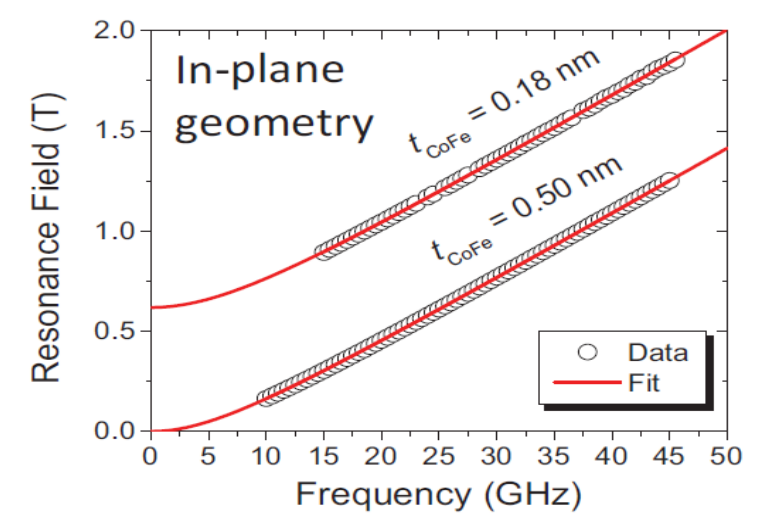

FIG. 14 (a) Schematic diagram of the multilayer and alloy sample structures. (b) Examples of imaginary and real parts of the FMR spectra taken at $30 \mathrm{GHz}$ for the $t_{\mathrm{CoFe}}=0.18 \mathrm{~nm}$ multilayer sample. The line through the data is the fit to Eq. (14). Exemplary Kittel plots of the resonance field versus frequency for the (c) perpendicular-to-plane and (d) in-plane geometries. The lines through the data are fits to Eqs. $(16,17)$. From [41].

\section{Peculiarities of the stripline FMR}

As we already mentioned, the cavity FMR is a well-established experimental technique and the interpretation of data obtained with it is relatively simple. However, the cavity FMR is very narrowband because of a very high $Q$-factor of the cavity.

In recent years the interest turned to the characterisation of the FMR line width. As the VNA FMR is a relatively new technique that has evolved in recent years, the interpretation of measured data (e.g., the accuracy of measurements of the resonance line width) is still sometimes a subject of discussions [46, 96, 99, 152, 153] because in the past measurements made on different setups did not yield identical results, largely due to differences in methodology or equipment limitations. In the VNA FMR, the scattering parameters $S_{11}$ and $S_{12}$ are measured. In this case, $S_{11}$ and $S_{12}$ contain information regarding both magnetic and non-magnetic properties of the sample. Knowing the $S$ parameters, the effective impedance arising from the magnetic response of the sample can be used in order to determine the effective susceptibility of the sample. To derive the change in the characteristic impedance caused by the magnetic response of the film, the dielectric properties of the film and the substrate must be known. In spite of the attempt to design the stripline with a near $50 \Omega$ impedance to match the VNA and cables, multiple reflections between the two ends of the sample must be considered. Hereafter, we discuss and compare several important raw data analysis techniques taking microwave reflection in the stripline into account. 
As suggested in Ref. [154], in order to take into account the effect of the reflections from the sample, the part of the stripline with the sample on top may be approximated as a lumped element with an effective inductance caused by the stripline itself and film susceptibility $(L)$, effective series resistance $(R)$, shunt conductance $(G)$, and capacitance $(C)$, caused by the stripline and the substrate of the sample. The equivalent device circuit is shown in Fig. 15. The reflection and transmission coefficients are

$$
\begin{array}{r}
S_{11}=\frac{i \omega L+R+\frac{Z_{0}}{1+Z_{0}(G+i \omega C)}-Z_{0}}{i \omega L+R+\frac{Z_{0}}{1+Z_{0}(G+i \omega C)}+Z_{0}}, \\
S_{21}=\frac{2 \frac{Z_{0}}{1+Z_{0}(G+i \omega C)}}{i \omega L+R+\frac{Z_{0}}{1+Z_{0}(G+i \omega C)}+Z_{0}},
\end{array}
$$

where $\omega$ is the microwave angular frequency and $Z_{0}$ is the characteristic impedance of the stripline. From Eqs. $(20,21)$ one obtains the simple relation

$$
i \omega L+R=\frac{1+S_{11}-S_{21}}{1-S_{11}} Z_{0} .
$$

In the particular permeameter setup described in Ref. [154] the static external magnetic field can be supplied either along or perpendicular to the stripline. When the sample is saturated along the hard axis, the microwave field is parallel/anti-parallel to the magnetisation vector, and thus $L=L_{\mathrm{w}}$, where $L_{\mathrm{w}}$ is the self-inductance of the stripline. The measured values are $S^{0}{ }_{11}$ and $S^{0}{ }_{12}$. When the magnetisation vector is along the easy axis with the microwave field perpendicular to it, noting that $\chi \approx \mu$, where $\chi$ and $\mu$ are relative susceptibility and relative permeability, the effective inductance is

$$
L \approx L_{\mathrm{w}}+\operatorname{clt} \mu \mu_{0}
$$

where $l$ is the length of the magnetic film of the sample, $t$ is the thickness of the film, $c$ is a geometry factor with a dimension $\mathrm{m}^{-1}$. Now the measured coefficients are $S^{1}{ }_{11}$ and $S_{12}^{1}$. The relative permeability can be written as

$$
\mu=\frac{Z_{0}\left(\frac{1+S_{11}^{1}-S_{21}^{1}}{1-S_{21}^{1}}-\frac{1+S_{11}^{0}-S_{21}^{0}}{1-S^{0}{ }_{21}}\right)}{i c l t \mu_{0} \omega} .
$$

The constant $c$ is typically determined by measuring a standard sample with known initial relative permeability. As a result, the complex relative permeability can be calculated without knowing the values of the other circuit components. 
(a)
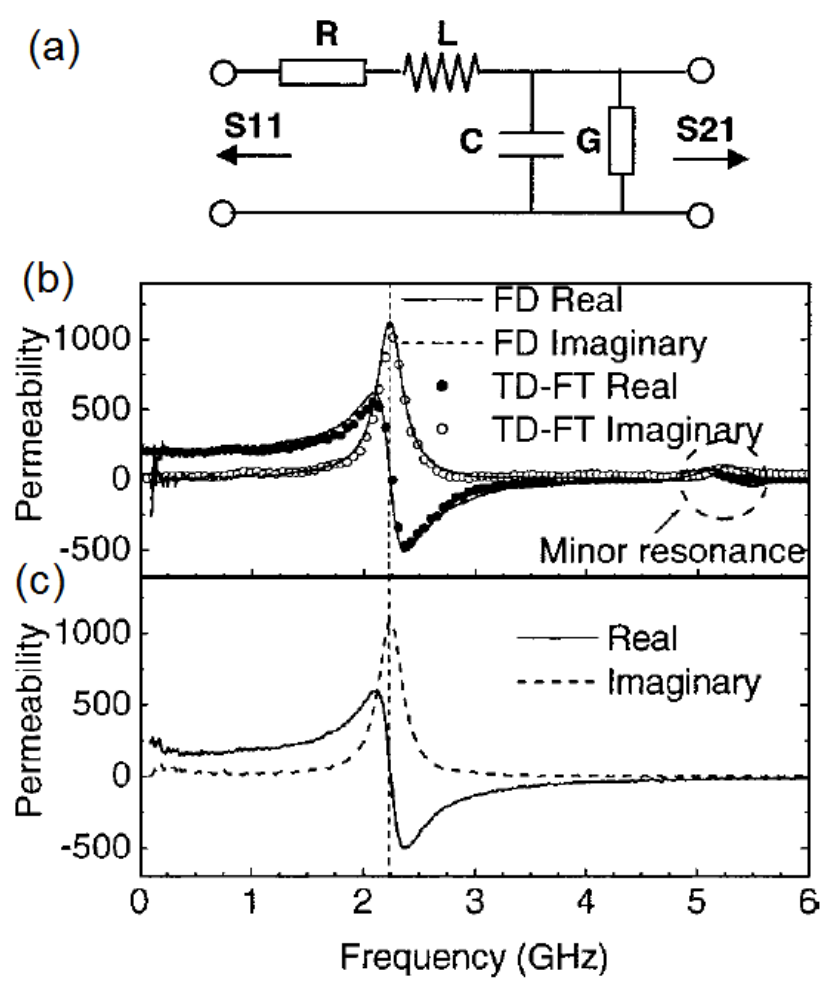

FIG. 15 (a) The equivalent circuit of a microstrip line section with a sample on the top is shown, where $L, R, C$, and $G$ are the effective inductance, series resistance, capacitance, and shunt conductance, respectively. $S_{11}$ and $S_{21}$ are the reflection and transmission coefficients. (b) Relative permeability of a Permalloy sample from microstrip frequency domain (solid and dotted lines) and time domain measurement (Fourier transform) (solid and open dots) with a longitudinal bias field of 50 Oe. (c) Relative permeability of the same sample from loop frequency domain measurement [154].

The solid and dashed lines in Fig. 15 show the real and imaginary parts of the relative permeability spectrum for a $100 \mathrm{~nm}$-thick Permalloy film at a bias field of 50 Oe. The circles in Fig. 15 show the real and imaginary parts of a Fourier transformed PIMM measurement for the same sample. One can see that the VNA FMR and the PIMM yield identical spectra. The close agreement between the frequency-domain (VNA-FMR) and time-domain (PIMM) measurements indicates that the $L R C$ equivalent circuit shown in Fig. 15 is a good approximation to the stripline with a magnetic film on the top. Furthermore, Fig. 15 shows equivalent data obtained using a loop permeameter, except the minor resonance corresponding to the first higher-order SSWM.

It is obvious that the different samples may have different dimensions and also the position of the sample with the respect to the axis of the stripline may vary (e.g. the sample can be shifted toward one of the input ports of the stripline.) This can affect the result of VNA FMR measurements leading to a considerable (up to $10 \%$ ) overestimation of the resonance line width [46]. To fix this problem, a full two-port data analysis technique was proposed in Ref. [46] to extract the resonance frequency and line width.

Let us assume that the stripline is connected to a VNA via two ports at the reference planes $R_{1}$ (left edge of the sample) and $R_{2}$ (right edge of the sample). A full two-port calibration has been performed. Thus, any contribution to the measured $S$-parameters from outside the stripline is compensated. The complex-valued $S$-matrix representing the section between the two ports is 
calculated analytically by multiplying several transmission matrices, followed by a transformation to the $S$-matrix.

$$
\begin{gathered}
T=\left(\begin{array}{cc}
e^{-\gamma_{0} l_{1}} & 0 \\
0 & e^{\gamma_{0} l_{1}}
\end{array}\right)\left(\begin{array}{cc}
\frac{1}{\sqrt{1-\Gamma^{2}}} & \frac{\Gamma}{\sqrt{1-\Gamma^{2}}} \\
\frac{\Gamma}{\sqrt{1-\Gamma^{2}}} & \frac{1}{\sqrt{1-\Gamma^{2}}}
\end{array}\right)\left(\begin{array}{cc}
e^{-\gamma l_{\mathrm{s}}} & 0 \\
0 & e^{\gamma l_{\mathrm{s}}}
\end{array}\right) \times \\
\\
\times\left(\begin{array}{cc}
\frac{1}{\sqrt{1-\Gamma^{2}}} & \frac{-\Gamma}{\sqrt{1-\Gamma^{2}}} \\
\frac{-\Gamma}{\sqrt{1-\Gamma^{2}}} & \frac{1}{\sqrt{1-\Gamma^{2}}}
\end{array}\right)\left(\begin{array}{cc}
e^{-\gamma_{0} l_{2}} & 0 \\
0 & e^{\gamma_{0} l_{2}}
\end{array}\right),
\end{gathered}
$$

where $l_{1} \neq l_{2}$ are the distances between the edges of the sample and the reference planes $R_{1}$ and $R_{2}$, respectively, and $l_{\mathrm{s}}$ the length of the sample. The five matrices multiplied in Eq. (25) represent: (i) the section of the stripline of length between the left port of the VNA and the sample edge (propagation constant $\gamma_{0}$ ); (ii) the change in the impedance from $Z_{0}$ to $Z$ at the left edge of the sample; (iii) the wave propagation in the loaded region of the stripline of length $l_{\mathrm{s}}$ with a modified propagation constant $\gamma$; (iv) the change in the impedance from $Z$ back to $Z_{0}$ at the right edge of the sample; and (v) the unloaded section of the stripline of length $l_{2}$ between the right edge of the sample and the right port.

In order for this description to be valid, the characteristic impedance of the stripline $Z_{0}$ needs to be equal to the characteristic impedance of the measurement system, e.g. $50 \Omega$. The impedance $Z$ of the stripline loaded with the sample is related to the complex reflection coefficient $\Gamma$ as follows

$$
\Gamma=\frac{Z-Z_{0}}{Z+Z_{0}} \Leftrightarrow \frac{Z}{Z_{0}}=\frac{1+\Gamma}{1-\Gamma} .
$$

Transformation of the $T$-matrix into an $S$-matrix results in the desired description of the stripline loaded with a magnetic sample

$$
S=\left[\begin{array}{cc}
e^{-\gamma_{0} 2 l_{1}} \frac{\Gamma\left(1-P^{2}\right)}{1-P^{2} \Gamma^{2}} & e^{-\gamma_{0}\left(l_{1}+l_{2}\right)} \frac{P\left(1-\Gamma^{2}\right)}{1-P^{2} \Gamma^{2}} \\
e^{-\gamma_{0}\left(l_{1}+l_{2}\right)} \frac{P\left(1-\Gamma^{2}\right)}{1-P^{2} \Gamma^{2}} & e^{-\gamma_{0} 2 l_{2}} \frac{\Gamma\left(1-P^{2}\right)}{1-P^{2} \Gamma^{2}}
\end{array}\right],
$$

where

$$
P=\exp \left(-\gamma l_{\mathrm{s}}\right)
$$

According to Eq. (27) the network is reciprocal, i.e. the two matrix elements $S_{12}$ and $S_{21}$ are equal within measurement accuracy. Consequently, only $S_{21}$ will be used. However, $S_{11}$ and $S_{22}$ are generally different, which is due to finite precision of centring of the sample between the ports $\left(l_{1} \neq l_{2}\right)$. This problem can be corrected mathematically by using a geometric mean of $S_{11}$ and $S_{12}$

$$
S_{11 / 22}=\sqrt{S_{11} \cdot S_{22}}=e^{-\gamma_{0}\left(l_{1}+l_{2}\right)} \frac{\Gamma\left(1-P^{2}\right)}{1-P^{2} \Gamma^{2}} .
$$

The transformation (29) makes the pre-factor $\exp \left[-\gamma\left(l_{1}+l_{2}\right)\right]$ the same for $S_{21}$ and $S_{11 / 22}$. This prefactor contains only the sum of the lengths of the two unloaded sections of the stripline, which equals to the total distance between the ports minus the sample length. In this way the effect of potential misalignment of the sample on the results of raw data processing becomes accounted for, as shown in Ref. [46]. 
In what follows, the reference planes are shifted from the positions of the ports connecting the stripline ( $R_{1}$ and $\left.R_{2}\right)$ to the VNA to the edges of the sample $\left(B_{1}\right.$ and $\left.B_{2}\right)$. This "de-embedding" is achieved by multiplying $S_{21}$ and $S_{11 / 22}$ with the inverse pre-factor. The latter is extracted by measuring $S_{21}$ of the stripline ${ }^{2}$ without the sample on top and not changing the positions $R_{1}$ and $R_{2}$. Since in the absence of the sample $S^{\mathrm{MS}}{ }_{21}=\exp \left(-\gamma_{0} l_{\text {total }}\right)$, the inverse pre-factor reads

$$
\left(e^{-\gamma_{0}\left(l_{\text {total }}-l_{\mathrm{s}}\right)}\right)^{-1}=\exp \left[-\frac{l_{\text {total }}-l_{\mathrm{s}}}{l_{\mathrm{s}}} \ln \left(S_{21}^{\mathrm{MS}}\right)\right] .
$$

The two de-embedded parameters are then given by

$$
\begin{aligned}
& S_{21}^{\mathrm{B}}=e^{\gamma_{0}\left(l_{\text {total }}-l_{\mathrm{s}}\right)} S_{21}=\frac{P\left(1-\Gamma^{2}\right)}{1-P^{2} \Gamma^{2}}, \\
& S_{11 / 22}^{\mathrm{B}}=e^{\gamma_{0}\left(l_{\text {total }}-l_{\mathrm{s}}\right)} S_{11 / 22}=\frac{\Gamma\left(1-P^{2}\right)}{1-P^{2} \Gamma^{2}} .
\end{aligned}
$$

As shown in Ref. [155], $\Gamma$ can be obtained from the measured $S$ parameters using

$$
\Gamma=K \pm \sqrt{K^{2}-1}
$$

with $K$ defined as

$$
K=\frac{1+\left(S_{11 / 22}^{\mathrm{B}}\right)^{2}-\left(S_{21}^{\mathrm{B}}\right)^{2}}{2 S_{11 / 22}^{\mathrm{B}}}
$$

The plus/minus sign before the square root in Eq. (33) is chosen such that $|\Gamma|<1$. The next step is computing $P$ (Eq. (28)) and extracting the propagation constant $\gamma$ from its value:

$$
\begin{gathered}
P=\frac{S_{11 / 22}^{\mathrm{B}}+S_{21}^{\mathrm{B}}-\Gamma}{1-\left(S_{11 / 22}^{\mathrm{B}}+S_{21}^{\mathrm{B}}\right) \Gamma}, \\
\operatorname{Re}(\gamma)=-\frac{\ln (|P|)}{l_{\mathrm{s}}} ; \operatorname{Im}(\gamma)=-\frac{\arg (\mathrm{P})}{l_{\mathrm{s}}} .
\end{gathered}
$$

It is worth noting that care has to be taken while evaluating the logarithm in (36) because $P$ is a complex number and the logarithm of a complex number is multi-valued. This can be resolved by calculating the real and imaginary parts of $\gamma$ separately [Eq. (36)], and taking care of possible $2 \pi$ jumps appearing in the complex argument of $P$. Our own experience shows that adding the appropriate number of $2 \pi$ to remove the jumps may be a problem when the measurements are taken applied field resolved for a given frequency (or frequencies). This problem is easily solved by taking an extra VNA sweep, this time a frequency sweep across the whole range of frequencies of interest for a given applied field (e.g. for a zero field). It is useful to start the sweep from the minimum VNA frequency. By adding $2 \pi n$ to the phase of $S_{21}, S_{22}$ and $S_{11}$ at the positions of the jumps in the respective raw phase vs. frequency dependence, the jumps can be removed to produce smooth curves. Usually, the wavelength of the microwave signal in the stripline for the lower-

${ }^{2}$ A coplanar line was used in Ref. [46]. However, their results should be applicable to the microstrip-line based BFMR as well. 
frequency edge of the VNA frequency range (typically 10 to $100 \mathrm{MHz}$ ) is significantly larger than the length of the stripline, therefore, for the lowest frequency jump one has $n=1$ and $n$ increases by one at each consecutive jump. The obtained values of $n$ for each frequency can be then used to correct the complex argument of $P$ in the field-resolved data.

The next step in the data evaluation procedure suggested in Ref. [46] is the introduction of an effective permittivity $\tilde{\varepsilon}_{\mathrm{r}}$ and an effective relative permeability $\tilde{\mu}_{\mathrm{r}}$. These effective quantities (marked by a tilde) are used to link the complex geometry, where several materials contribute to $\tilde{\varepsilon}_{\mathrm{r}}$ and $\tilde{\mu}_{\mathrm{r}}$, to an effective medium model where the stripline is surrounded entirely by one single material with these effective electromagnetic properties. $\tilde{\varepsilon}_{\mathrm{r}}$ and $\tilde{\mu}_{\mathrm{r}}$ are related to $\Gamma$ and $\gamma$ by

$$
\begin{aligned}
& \frac{\gamma}{\gamma_{\mathrm{fs}}}=\frac{\gamma}{i 2 \pi f \sqrt{\mu_{0} \varepsilon_{0}}}=\sqrt{\tilde{\mu}_{\mathrm{r}} \tilde{\varepsilon}_{\mathrm{r}}} \\
& \frac{1+\Gamma}{1-\Gamma}=\frac{Z}{Z_{0}}=G \sqrt{\frac{\tilde{\mu}_{\mathrm{r}}}{\tilde{\varepsilon}_{\mathrm{r}}}} \propto \sqrt{\frac{\tilde{\mu}_{\mathrm{r}}}{\tilde{\varepsilon}_{\mathrm{r}}}} .
\end{aligned}
$$

The variable $\gamma_{\mathrm{fs}}$ is the propagation constant of the stripline in free space, and $G$ is a frequencyindependent constant depending on the geometry of the stripline. Because $G$ is difficult to estimate, one can easily calculate values proportional to effective quantities

$$
\tilde{\mu}_{\mathrm{r}} \propto \frac{\gamma}{\gamma_{\mathrm{fs}}} \frac{1+\Gamma}{1-\Gamma} ; \tilde{\varepsilon}_{\mathrm{r}} \propto \frac{\gamma}{\gamma_{\mathrm{fs}}} \frac{1-\Gamma}{1+\Gamma} .
$$

Whereas the main interest is in permeability, it is still important to evaluate the effective relative permittivity. In this case, it is impossible to separate the different contributions from the relative permittivity $\varepsilon_{\mathrm{r}}$ of the substrate of the stripline, the substrate of the sample, and of the small air gap between the sample and the stripline. However, it Ref. [46] it was shown that $\operatorname{Im}\left(\tilde{\varepsilon}_{\mathrm{r}}\right)<<\operatorname{Re}\left(\tilde{\varepsilon}_{\mathrm{r}}\right)$ and that $\operatorname{Re}\left(\tilde{\varepsilon}_{\mathrm{r}}\right)$ is a constant over the frequency range of interest. Hence, this simplifies the calculation of $\tilde{\mu}_{\mathrm{r}}$ as

$$
\frac{\gamma}{\gamma_{\mathrm{fs}}} \propto \tilde{\varepsilon}_{\mathrm{r}} \frac{1+\Gamma}{1-\Gamma} \Rightarrow \tilde{\mu}_{\mathrm{r}} \propto\left(\frac{1-\Gamma}{1+\Gamma}\right)^{2} ; \tilde{\mu}_{\mathrm{r}} \propto\left(\frac{\gamma}{\gamma_{\mathrm{fs}}}\right)^{2} .
$$

Using Eq. (40) instead of Eq. (39) offers an advantage because only one of the two extracted quantities $(\Gamma$ or $\gamma$ ) is required. Consequently, depending on specific measurement conditions, one of these quantities can be determined with higher precision [156]. For instance, at frequencies up to a few $\mathrm{GHz}$ low noise is observed in both $\Gamma$ and $\gamma$. However, above $10 \mathrm{GHz}$ the noise in $\Gamma$ increases significantly, but it stays low in $\gamma$.

It is also worth noting that $\tilde{\mu}_{\mathrm{r}}$ is superposition of the relative permeability $\mu_{\mathrm{r}}$ of the magnetic layer of the sample and the relative permeability of the other surrounding material (which is equal to one). As $\tilde{\mu}_{\mathrm{r}}$ is an unknown linear function of $\mu_{\mathrm{r}}$ of the magnetic material, a value directly proportional to the transverse susceptibility $\chi$ can be introduced

$$
\chi=\mu_{\mathrm{r}}-1 \propto \tilde{\mu}_{\mathrm{r}}-\tilde{\mu}_{\mathrm{r}}^{\mathrm{ref}},
$$

where $\tilde{\mu}_{\mathrm{r}}$ is the measurement result (the magnetic field is typically orientated along the stripline) and $\tilde{\mu}_{\mathrm{r}}$ ref is the result of a reference measurement for which the magnetic field is orientated perpendicular 
to the stripline (and parallel to the excitation field), and hence no FMR is excited.

In Ref. [46] results obtained using the described data evaluation technique were compared to those obtained with the other evaluation schemes $[152,157]$. It was found that whereas the FMR frequencies obtained by the different methods agree well (the relative error $<1 \%$ ), the resonance line width differ up to $10 \%$.

BFMR measurements can be also affected by the presence of tiny amount of magnetic material in the end-launch connectors and $L$-shape connectors used to connect the stripline to microwave cables, as well as in the stripline itself. This introduces some experimental error. It can be quantified as $\mathrm{S} 21_{0}(H)$ - the field dependence of the $\mathrm{S} 21$ parameter of the microstrip line without a sample on its top. It was shown that $\ln \left[\mathrm{S} 21 / \mathrm{S} 21_{0}\right]$ scales as the complex microwave impedance inserted by the presence of the sample on top of the stripline. Accordingly, the real part of $r^{\prime}=\mathrm{S} 21(H) / \mathrm{S} 21_{0}(H)$ should have a Lorentzian (bell) shape characteristic to the real part of the impedance or imaginary part of $\chi \cdot \operatorname{Im}\left(r^{\prime}\right)$ is described by a sign varying function featuring a characteristic $\pi$ phase jump at the resonance frequency, characteristic to the resonance induced reactance or $\operatorname{Re}(\chi)$.

Experimental data, however, often display deviation from this picture - they frequently look as linear combination of these "standard" curves. Observations show that in the general case the experimentally measured real part has a shape as follows: $C_{1} \operatorname{Re}\left(r^{\prime}\right)+C_{2} \operatorname{Im}\left(r^{\prime}\right)$. The measured imaginary part of $\mathrm{S} 21$ can be cast in a similar form: $-C_{2} \operatorname{Re}\left(r^{\prime}\right)+C_{1} \operatorname{Im}\left(r^{\prime}\right)$. The coefficients $C_{1}$ and $C_{2}$ of these linear combinations are real numbers and $\left|C_{1}+i C_{2}\right|=1$. This demonstrates that $C_{1}=\cos (\Delta \phi)$ and $C_{2}=\sin (\Delta \phi)$, where $\Delta \phi$ is some phase shift. It appears that $\Delta \phi$ is not arbitrary, but is usually in good agreement with the formula as follows $\Delta \phi=\arg \left[\mathrm{S} 21\left(H_{\max }\right) / \mathrm{S} 21_{0}\left(H_{\max }\right)\right]$. Here $H_{\max }$ is the maximum field accessible in the experiment (which serves here as a proxy to an infinitely large field), and "arg" denotes the phase of the respective complex number. Introduction of this phase shift produces a new complex trace: $\quad r=\left|\mathrm{S} 21(H) / \mathrm{S} 21_{0}(H)\right| \times \exp (i \Delta \phi)$, where $i \Delta \phi=\arg \left[\mathrm{S} 21(H) / \mathrm{S} 21_{0}(H)\right]-\arg \left[\mathrm{S} 21\left(H_{\max }\right) / \mathrm{S} 21_{0}\left(H_{\max }\right)\right]$. The new dependencies $\operatorname{Re}(r)$ and $\operatorname{Im}(r)$ on $H$ have the shapes which are very close to the standard ones. For wide microstrip FMR transducers, as used in the experiment [88], $\operatorname{Im}(r)$ is often much smaller than $\operatorname{Re}(r)$. This results in a very convenient and compact form for the output data, since $\operatorname{Im}(r)$ may be neglected.

As a final note we would like to emphasise that the length of the coplanar line used for the measurements of $S_{21}$ and $S_{11}$ in [46] was quite short: just $5 \mathrm{~mm}$ [96]. For longer striplines (which are more common), one may expect larger uncertainty in the extraction of $\tilde{\mu}_{\mathrm{r}}$ from the raw data, because for the longer striplines, the stripline length is more comparable with the wavelength of the electromagnetic wave in it and uncertainties of measurements of the phases of $S_{11}, S_{22}$, and $S_{22}$ may contribute more to the results of calculations using Eqs. $(34,35)$. Also we would like to note that the same authors have also developed a method of extraction of $\tilde{\mu}$ from single-port measurements of $S_{11}$ [158].

\section{Most general peculiarities of magnetisation dynamics arising from driving it by the microwave field of striplines}

\subsection{Dependence of the BFMR response on the stripline width and the travelling spin wave contribution}


BFMR response depends on the distribution of the microwave magnetic and electric fields created by the stripline. The distributions of these fields depend on the geometry of the particular stripline and the material properties of the sample under test, which sits on top of this stripline. A detailed discussion of the effect of the sample material on the stripline FMR response is presented in Sections 5.

BFMR response also depends on the amplitudes of the microwave magnetic and electric fields. Hence, the most favourable experimental position of the sample is when the magnetic film faces the stripline. In this configuration, the magnetic film is physically closer to the line and the amplitude of the microwave magnetic field induced in the film is the maximum. However, as discussed in Section 5.3.2, one can also place the sample such that the substrate of the magnetic film faces the stripline. Of course, in this configuration the amplitude of the microwave magnetic field reaching the magnetic film is lower.

Below we will consider the example of a microstrip line. However, the results are equally applicable to a coplanar-line BFMR transducer as well, provided the width of the microstrip is replaced by some characteristic width of the coplanar line in the formulas. The sum of the width of the signal line and of the widths of the two gaps between the signal and the ground line is usually a good candidate to the characteristic width [88].

Let us assume that the thickness (the perpendicular-to-plane size) of the microstrip ${ }^{3}$ is negligibly small and that the total microwave current $I$ is uniform across the microstrip width (the microstrip size in its plane) $w$. Then the linear current density in the microstrip is $j=I / w$. As follows from Maxwell equations, in the close vicinity of the microstrip the in-plane component of the microwave magnetic field is $j / 2$. The amplitude of magnetisation precession in the sample placed close to the microstrip scales as the driving field and hence as $j$. We are able to detect the onset of FMR in the material because the sample provides feedback to the microstrip in the form of a microwave electric field induced by the precessing magnetisation vector. The amplitude of this electric field at the position of the microstrip scales as the precession amplitude and hence as $j$. Importantly, it does not depend on $w$. As a result, the amplitude of the FMR peak at the output of the microstrip line scales as $j=U /\left(w Z_{0}\right)$, where $Z_{0}=50 \Omega$ is the characteristic impedance of the microstrip line and $U$ is the microwave voltage supplied by the microwave generator. From the last formula one sees that decreasing $w$ should improve the signal-to-noise ratio for the stripline FMR setup.

Narrow striplines are also needed for the studies of nonlinear FMR dynamics [159, 160]. Given very large magnetic losses for metallic ferromagnets, the oscillating magnetic field $h_{\mathrm{rf}}$ needed to produce nonlinear behaviour in metallic magnetic films ranges from 35 to 230 Oe.

\footnotetext{
${ }^{3}$ In the theoretical analysis below we neglect the effect of mirror current flowing along the ground plane of the microstrip line and consider only the microwave current flowing in the microstrip (s ee Fig. 6(a) for the explanation of the notions "microstrip" and "ground plane"). The mirror currents were included in the recent theory [161].
} 

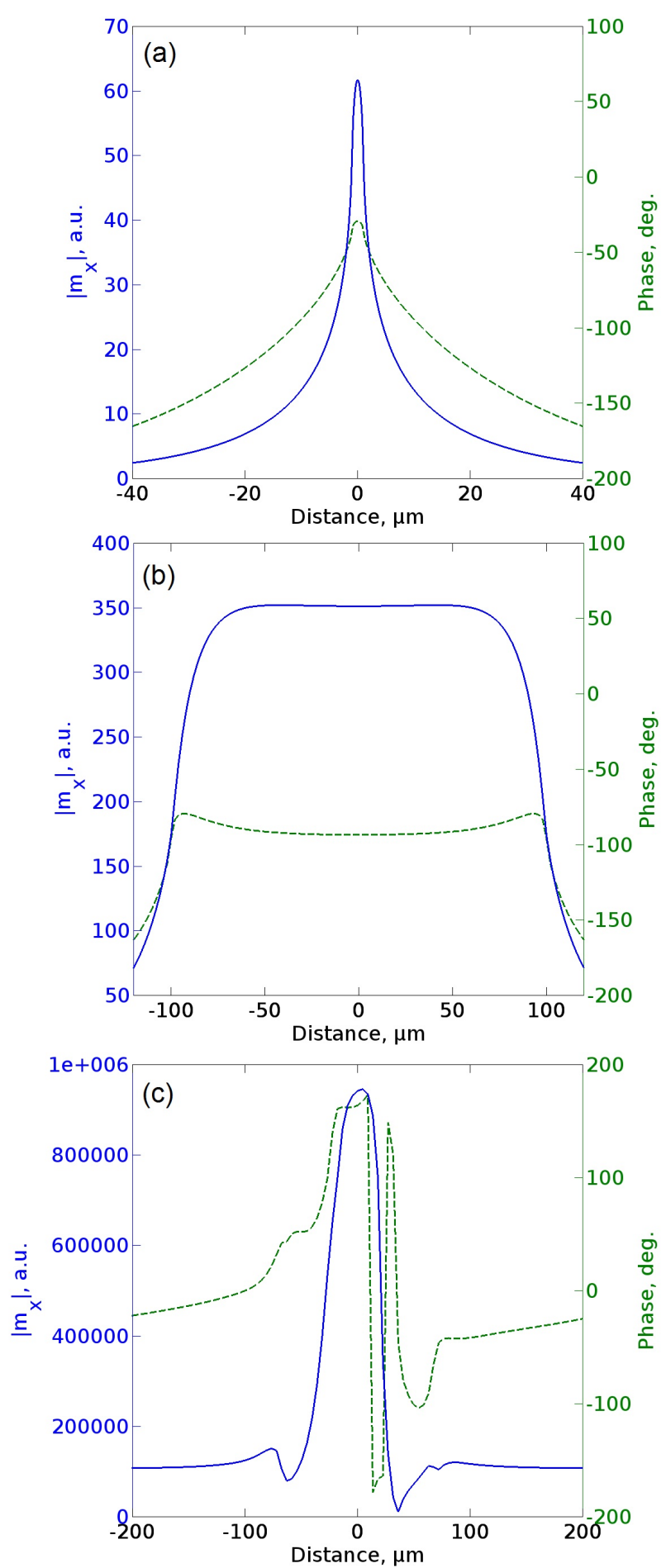

FIG. 16 Simulated in-plane dynamic magnetisation as function of distance from the microstrip line symmetry axis. Blue solid line - the magnitude of the in-plane magnetisation $\left|m_{\mathrm{x}}\right|$, green dashed line - phase $\arg \left(m_{\mathrm{x}}\right)$. The microstrip width is (a) $2 \mu \mathrm{m}$ and (b) $200 \mu \mathrm{m}$. The film thickness is $110 \mathrm{~nm}$, saturation magnetisation is $4 \pi M_{\mathrm{s}}=8900 \mathrm{G}$, and the microwave frequency is $10 \mathrm{GHz}$. The static magnetic field is applied perpendicular to the film plane. (c) The same but for the geometry of the in-plane BFMR utilising a coplanar line. The coplanar line characteristic size is the same as in the experiment from [96] - the width of the signal line is $45 \mu \mathrm{m}$ and the film thickness is $50 \mathrm{~nm}$. The width of the gaps between the signal and the ground line is assumed to be the same $-45 \mu \mathrm{m}$. 
However, there is an important limitation to the minimum stripline width. In contrast to the cavity FMR, where the microwave magnetic field is perfectly uniform, the microwave magnetic field of a stripline is non-uniform on the length scale given by $w$. This spatially non-uniform field can potentially excite travelling spin waves in the film plane. The excited wave vector range is $0<1$ $k \mid<2 \pi / w$, being $w$ the width of the microstrip. (For a coplanar line $k=2 \pi /(w+\Delta)$, where $\Delta$ is the separation of the central conductor from the ground planes [88].) The direction of $\mathbf{k}$ is perpendicular to the microstrip and the waves are excited in both directions $(+k$ and $-k)$. The excitation of travelling spin waves takes place if the lateral dimensions of the sample are much larger than the free propagation path of spin waves $l_{\mathrm{f}}$, and if the width of the microstrip is comparable to $l_{\mathrm{f}}$. Following Ref. [88], the free propagation path scales as the magnetic losses in the material expressed in the form of the frequency resolved FMR line width $\Delta \omega$. It also scales as $1 / V_{\mathrm{g}}$, where $V_{\mathrm{g}}$ is the spin wave group velocity.

Consider the typical example of the IP FMR. In this case the applied field is parallel to the stripline and the type of spin waves which is excited in this situation is the Damon-Eshbach Magnetostatic Spin Wave (DE MSSW) [9]. The group velocity of DE MSSW for small wave numbers scales as the film thickness $L$ and the film saturation magnetisation [43]

$$
\frac{1}{V_{g}}=\frac{4}{\omega_{\mathrm{M}} L} \frac{\sqrt{\omega_{\mathrm{H}}\left(\omega_{\mathrm{H}}+\omega_{\mathrm{M}}\right)}}{\omega_{\mathrm{M}}},
$$

where $\omega_{\mathrm{H}}=|\gamma| \mu_{0} H$ and $\omega_{\mathrm{M}}=|\gamma| \mu_{0} M_{\mathrm{s}}$. The free propagation path is $l_{\mathrm{f}}=V_{\mathrm{g}} /\left(\omega \alpha_{\mathrm{G}}+\gamma \Delta H_{0}\right)$ where $\omega \alpha_{\mathrm{G}}+\gamma \Delta H_{0}$ is the frequency-resolved FMR line width. From this expression, for $f=10 \mathrm{GHz}$ and $4 \pi M_{\mathrm{s}}=10500 \mathrm{G}$ one obtains $l_{\mathrm{f}}=30 \mu \mathrm{m}$ for an $L=100 \mathrm{~nm}$ Permalloy film $\left(\alpha_{\mathrm{G}}=0.008, \Delta H_{0}=0\right)$ and $l_{\mathrm{f}}=0.62 \mu \mathrm{m}$ for $L=2 \mathrm{~nm}$.

The same applies to the case of the PP FMR. In this case the Forward Volume Magnetostatic Spin Waves are excited [162]. In Fig. 16(a,b), we plot the amplitude and phase of FVMSW as a function of the distance from the microstrip carrying a microwave current. This numerical result was obtained by using the theory from [163]. One sees a sharp maximum of the magnetisation precession amplitude right beneath the transducer and an exponential decay of the precession amplitude with the distance from it. Together with a gradual change of the phase of precession with the distance, the spatial decay of the precession amplitude evidences the excitation of propagating spin waves. The spin wave amplitude decays exponentially due to the losses in the medium [Fig. 16(a)]. The phase is accumulated linearly because it scales as $|k d|$, where $d$ is the distance from the edge of the microstrip. The distance which spin waves can travel before they decay completely is $3 l_{\mathrm{f}}$.

The spin waves propagate in both directions from the microstrip transducer and carry away the energy of the "resonance" which takes place below the transducer. This results in a broadening of the FMR peak [96]. One may think of the area in Fig. 16 right below the transducer, where the phase and the amplitude are almost constant as the area where the resonant FMR absorption takes place. The edges of this area are essentially the edges of the microstrip. From the comparison of the panels (a) and (b) of Fig. 16 one sees that with an increase in the transducer width the resonant absorption area becomes larger with respect to $3 l_{\mathrm{f}}$. As a result, for transducers which are much wider than $3 l_{\mathrm{f}}$ (as in Fig.16(b)) the spin wave contribution to the resonance line width becomes negligible. 
The (in-plane) profile of the magnetisation amplitude across the resonant absorption area in Fig. 16(b) mimics the profile of the microwave magnetic field of the microstrip line. This represents one more reason for which one may consider the microwave energy absorption in this area as the FMR absorption. It is worth noting that the microwave magnetic field of real microstrip and coplanar lines is more non-uniform in the plane. This is due to the strong repulsion of the microwave currents. This property is an in-plane counterpart of the usual microwave skin effect. As a result, the microwave current density tends to strongly concentrate at the stripline edges. Given the electromagnetic boundary condition for the field of a thin sheet of a current $h_{\mathrm{x}}=j_{\mathrm{z}} / 2$, the microwave field profile along the width of the stripline follows closely the distribution of the current density (see Fig. 6).

The capability of striplines to excite propagating spin waves in a broad range of frequencies explains why in Propagating Spin Wave Spectroscopy (PSWS) experiments one uses micrometresized spin wave antennas $[12,163,164]$. Figure 17 schematically shows the dispersion relation of magnetostatic surface spin waves (MSSW) [9] and the spectrum of Fourier-components for the microwave field created by a microstrip line. The DE MSSW propagates at a right angle to the magnetic field applied in the film plane. This is precisely the geometry of the in-plane stripline FMR.

Figure 16(c) shows the profile of the dynamic magnetisation calculated for the coplanar line geometry used in the in-plane BFMR experiment from [96]. The data in Fig. 16(c) correspond to the applied field value which differs by $\Delta H / 2$ from the FMR field, being $\Delta H$ the intrinsic fieldresolved resonance line width for the fundamental FMR mode. One observes a significant asymmetry of the profile of the dynamic magnetisation, which is a fundamental signature of the Damon-Eshbach geometry - a strong non-reciprocity of MSSW excitation by striplines. This asymmetry is due to the fact that both IP and PP components of the microwave Oersted field of the current in the stripline contribute to the excitation [89, 163]. Hence, the asymmetric profile in Fig. 16(c) evidences a significant contribution of the PP component of the microwave field to the formation of the stripline FMR response. This represents one more important peculiarity of the stripline FMR with respect to the conventional cavity FMR: the microwave field in the centre of a cavity is perfectly parallel to the cavity walls. Therefore, the magnetisation precession is driven only by the IP component of the microwave field in this case. Below in Sections 4.1 and 7.4 we will present experimental evidence of the importance of PP microwave field component in some particular cases.

Another important observation from Fig. 16(c) is a strong non-uniformity of both amplitude and phase of the dynamic magnetisation. This profile is consistent with potential presence of propagating spin waves in the gap between the signal and ground lines. It is not easy to quantitatively estimate the contribution of propagating spin waves to the total BFMR response in this case. However, the analytical theory by Counil et. al. [96] showed that this contribution exists for these particular coplanar sizes.

Let us now discuss the origin of the resonance line width broadening from a different perspective. If we use the same model of the uniform distribution of the microwave current density across the stripline width as above, we obtain $\left|\mathbf{h}_{\mathrm{ek}}\right|=\left|j_{\mathrm{k}}\right| / 2=\sin (k w / 2) /(k w / 2)$, being $k$ the wave vector and $j_{\mathrm{k}}$ the Fourier-transform of the microwave current density distribution [166]. (For a coplanar line the Fourier spectrum is more complex, see Fig. 25(a).) The first zero of $\left|\mathbf{h}_{\mathrm{ek}}\right|$ is located at $k=2 \pi / w$. For two striplines of widths $w_{1}$ and $w_{2}\left(w_{2}<w_{1}\right)$ the first zero is denoted by $k_{1}$ and $k_{2}$, 
respectively [Fig. 17(b)]. One sees that the wider stripline $\left(w_{1}\right)$ excites the spin wave modes in the range $\delta \omega_{1}=\omega_{1}-\omega_{0}$, being $\omega_{0}$ the FMR frequency. The narrower stripline $\left(w_{2}\right)$ excites the spin wave modes in the range $\delta \omega_{2}=\omega_{2}-\omega_{0}, \delta \omega_{2}>\delta \omega_{1}$. As a criterion for the choice of the width of the stripline for the BFMR one can use the inequality $\gamma \Delta H>>\delta \omega$. In contrast, for the efficient excitation of the propagating spin wave modes the $\gamma \Delta H<<\delta \omega$ criterion should be satisfied.

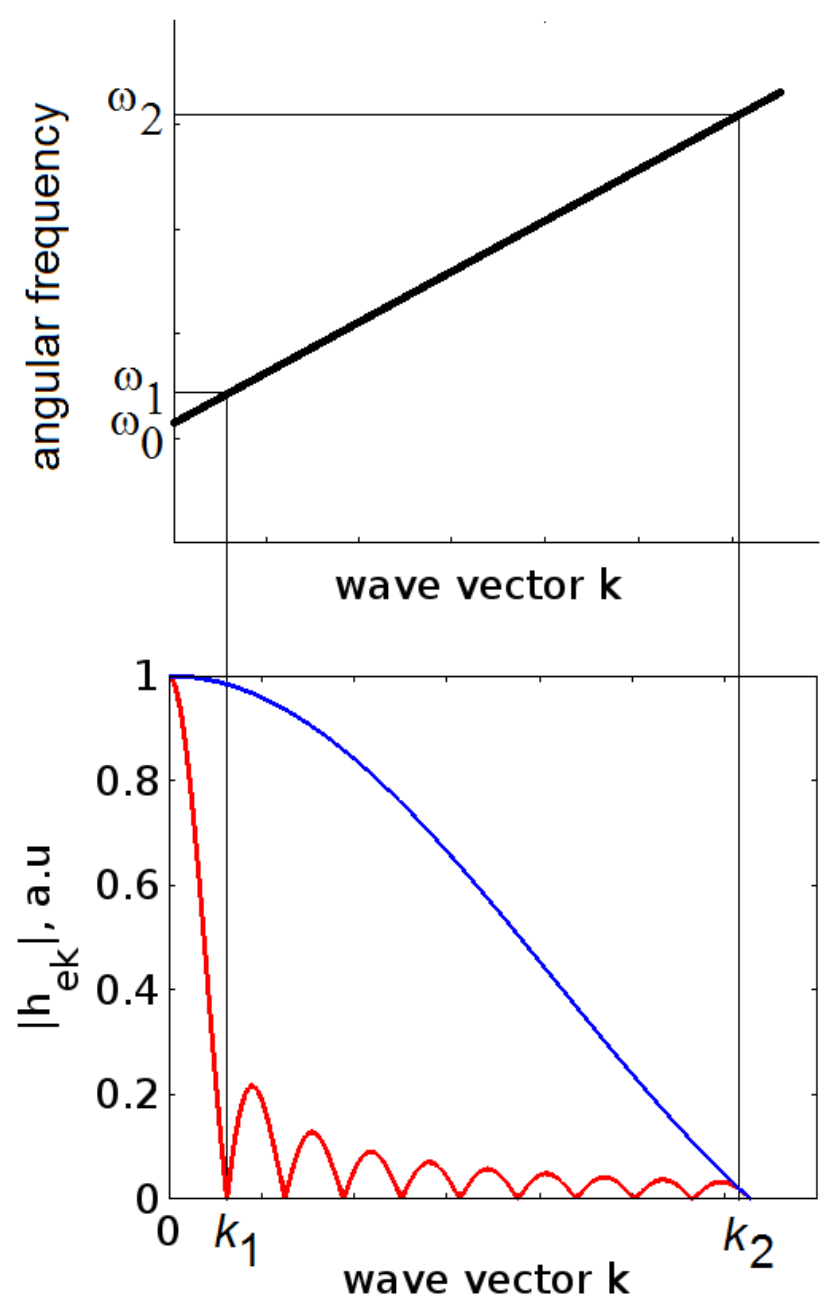

FIG. 17 (a) Illustration of the dispersion relation of the magnetostatic spin wave modes. (b) Spectrum of Fourier-components for the microwave field created by two striplines of widths $w_{1}$ (red line) and $w_{2}$ (blue line, $w_{2}<w_{1}$ ). The first zero of the spectrum is denoted by $k_{1}$ and $k_{2}$, respectively. (In this sketch, we show the spin wave dispersion with a positive slope. Accordingly, this picture is valid for Damon-Eshbach [9] and forward volume spin wave configuration [162]).

This idea is illustrated in Fig. 18, in which we present results of the calculation of the microwave power reflected from a microstrip line loaded with a thin Permalloy film. The calculation is conducted for two widths of the microstrip line. In this simulation, we use the theory from [163]. One of the ends of the microstrip line is shorted, which is a proxy to the single-port stripline BFMR method from [46]. However, one may expect a very similar behaviour for the standard stripline BFMR operating in the transmission mode (2-port FMR).

From Fig. 18 one clearly sees that for the $20 \mu \mathrm{m}$-wide stripline [Fig. 18(a)] the field- 
resolved absorption band is larger and the resonance line shape is noticeably asymmetric. This is because the absorption line corresponds to the excitation of the DE wave in the wave-number range from zero to $\sim 3200 \mathrm{~cm}^{-1}$. The propagating spin waves contribute to the formation of the lower-field slope of the absorption line (see Fig. 17(a), but note that in Fig. 17(a) the MSSW dispersion is plotted frequency resolved and the data in Fig. 18 are plotted applied-field resolved ${ }^{4}$ for a given frequency). At the fields above the resonance field, the propagating MSSW do not exist and the higher-field slope of the resonance line is due to the magnetic losses in the medium. Therefore, its shape is very close to the Lorentzian one.

For the $200 \mu \mathrm{m}$-wide microstrip line the line width is significantly smaller, and the line shape is more symmetric and closer to a Lorentzian one. This is because the range of spin-wave wave numbers excited in this case is from 0 to $\sim 320 \mathrm{~cm}^{-1}$. This $k$-range corresponds to the $\sim 1047 \div 1063$ Oe applied-field range in the applied-field resolved spin wave dispersion. Importantly, this range is smaller $(\sim 16 \mathrm{Oe})$ than the intrinsic FMR line width $\Delta H$.
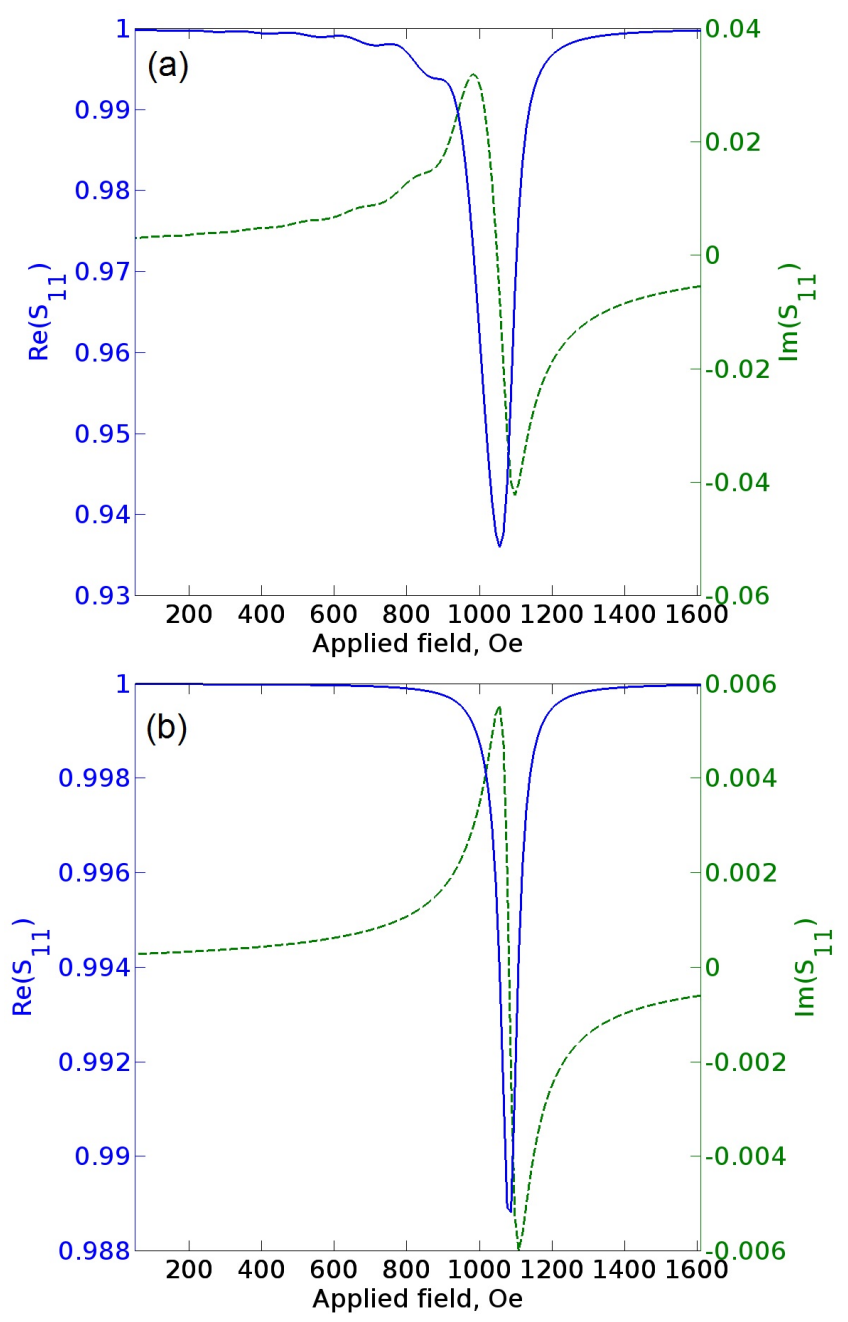

FIG. 18 Simulated $S_{11}$ for two microstrip widths. (a) $w=20 \mu \mathrm{m}$. (b) $w=200 \mu \mathrm{m}$. The film thickness is $110 \mathrm{~nm}$. The microwave frequency is $10 \mathrm{GHz}$. Film saturation magnetisation: $11100 \mathrm{G}$. Intrinsic resonance linewidth: $\Delta H=25$ Oe (full linewidth at half-maximum: $2 \Delta H=50 \mathrm{Oe}$ ).

\footnotetext{
4"Applied-field resolved dispersion" means the dependence of $k$ on the applied field for a fixed microwave frequency.
} 
Based on this simple physical picture, Counil et. al. [96] derived analytical expressions that make it possible to separate the extrinsic contribution to the FMR peak width originating from excitation of spin waves having non-zero in-plane wave vector $k$. The total absorption can be obtained by summation over all contributions from the excited spin waves

$$
\chi_{\text {tot }}^{\prime \prime}(\omega) \propto \int_{0}^{k_{\max }} \frac{\rho(k) d k}{1+\left\{\frac{2\left[\omega-\omega_{\mathrm{s}}(k)\right]}{\Delta \omega_{\text {int }}}\right\}^{2}},
$$

where $k_{\max }=2 \pi / w, \rho$ represents the constant density of excitations in the wave vector space, $\omega_{\mathrm{s}}(k)$ is the dispersion law for spin waves, and $\Delta \omega_{\text {int }}$ is the "intrinsic" line width corresponding to the "intrinsic" damping factor $\alpha_{0}$ given by $\Delta \omega_{\text {int }}=\alpha_{0} \gamma \mu_{0} M_{\text {eff. }}$ As shown in Ref. [96], in the considered particular geometry only spin waves with the in-plane wave vector $k$ in the $x$-direction may be excited. Hence, the relevant density of excitations is one-dimensional in the wave vector space, i.e. $\rho(\omega)=\rho(\partial \omega / \partial k)^{-1}$. One can sum up the contribution of each mode in the frequency space as

$$
\chi_{\text {tot }}^{\prime \prime}(\omega) \propto \int_{\omega_{0}}^{\omega\left(k_{\max }\right)} \frac{d \omega}{1+\left\{\frac{2\left[\omega-\omega_{\mathrm{s}}(k)\right]}{\Delta \omega_{\text {int }}}\right\}^{2}},
$$

where $\omega_{0} \equiv \omega(k=0)$ is the FMR frequency. From Eq. (44) one obtains the expressions for the peak frequency shift $\delta \omega_{0}\left(k_{\max }\right)$ and for the total line width $\Delta \omega\left(k_{\max }\right)$. In the limit of small dispersion in wave vector space these expressions read

$$
\begin{aligned}
& \delta \omega_{0}\left(k_{\max }\right) \approx \frac{\omega_{\mathrm{M}} \omega_{\mathrm{Meff}}}{8}\left(\frac{k_{\max } d_{\text {film }}}{\omega_{0}}\right) \\
& \Delta \omega\left(k_{\max }\right) \approx \Delta \omega_{\mathrm{int}}+C\left(\frac{k_{\max } d_{\text {film }}}{\omega_{0}}\right)^{2},
\end{aligned}
$$

where $d_{\text {film }}$ is the thickness of the magnetic film, $C$ is a constant depending on $\omega_{\mathrm{M}}=\gamma \mu_{0} M_{\text {eff }}$ and $\Delta \omega_{\text {int }}$, being $\mu_{0} M_{\text {eff }}=M_{\mathrm{S}}-2 K_{\mathrm{S}} /\left(M_{\mathrm{S}} d_{\text {film }}\right)$ the effective demagnetising field (determined as the field applied normally to the sample surface necessary to saturate the sample) and $K_{\mathrm{S}}$ the surface anisotropy. Once these expressions have been obtained, it is possible to separate the extrinsic contribution of propagating spin waves $\alpha_{\text {ext }}\left(k_{\max }\right)$ and the "intrinsic" damping factor $\alpha_{0}$

$$
\alpha_{\text {tot }}\left(k_{\max }\right) \approx \frac{\Delta \omega_{\text {int }}}{\omega_{M_{\text {eff }}}}+\frac{C}{\omega_{M_{\text {eff }}}}\left(\frac{k_{\max } d_{\text {film }}}{\omega_{0}}\right)^{2} \equiv \alpha_{0}+\alpha_{\text {ext }}\left(k_{\max }\right) .
$$

From the expressions above one sees that the resonance frequency shift $\delta \omega_{0}$ scales linearly with the film thickness [see the first equation of Eqs. (45)] and $1 / \omega_{0}$ (see Fig. 6 in [96]). This scaling is consistent with experimental data collected by Counil et al. [96] for $50 \mathrm{~nm}$ and $10 \mathrm{~nm}-$ thick Permalloy films. Experimentally Counil et al. found that at small frequencies $(1-2 \mathrm{GHz})$ for the $50 \mathrm{~nm}$-thick film $\alpha_{\text {tot }}$ is between 0.02 and 0.012 and drops to the intrinsic value $\alpha_{0}=0.0062$ at 6 $\mathrm{GHz}$ or so (Fig.8 in [96]). For the $6 \mathrm{~nm}$-thick film no noticeable experimental frequency dependence 
of $\alpha$ was found (see Fig. 7 in [96]), which is also in agreement with Eq. (46).

Finally, we would like to note that Eqs. (43)-(46) were derived assuming that the dispersion curve represents a straight line for small wave vectors $k$. However, as shown in [167], in ferromagnetic metal films the dispersion curve may deviate from a straight line due to the impact of eddy currents.

\subsection{Contribution of the PP component of the microwave magnetic field of striplines}

As sketched in Fig. 6(a, b), the microwave currents in coplanar and microstrip lines generate significant PP components of the microwave magnetic field. Thus, a sample located in close vicinity to the surface of a stripline experiences an effect of both IP and PP components of the microwave magnetic field. It is clear that the PP component cannot contribute to the formation of the FMR response in the case of PP BFMR, because this component is parallel to the applied field. However, in the case of the IP BFMR both components are perpendicular to the applied field and potentially they can deliver equal or similar contributions to the total FMR response of a material.

As we mentioned in the previous section, the asymmetry of the dynamic magnetisation profile in Fig. 16(c) is due to the fact that both PP and IP components contribute to the profile shape. The asymmetry arises because from one side of the vertical symmetry axis of the stripline [see Fig. 6(a) and (b)] the contributions of the two components sum up in phase, but from the other side of the axis they sum up in anti-phase [89]. However, this asymmetry is not very pronounced, and below we will show that this is because of the stronger contribution of the PP component of the microwave magnetic field to the FMR absorption in planar geometries.

Consider a continuous film magnetised in its plane. Then from Eq. (1) for the conditions of the IP ferromagnetic resonance we easily find that $\mathbf{m}=\hat{\kappa}\left(\mathbf{h}+\mathbf{h}_{d}\right)$, being $\mathbf{m}$ the vector of the dynamic magnetisation, $\mathbf{h}$ is the microwave driving field, and $\mathbf{h}_{\mathrm{d}}$ is the dynamic dipole (or demagnetising field) created by the dynamic magnetisation. For the IP geometry $\mathbf{h}_{\mathrm{d}}$ has only a PP component. It equals precisely to the PP component of $\mathbf{m}$, but has an opposite sign. Above, $\hat{\kappa}$ is the microwave magnetic susceptibility tensor [43].

Let us consider the in-plane component of the dynamic magnetisation $m_{\mathrm{x}}$. One finds that $m_{x}\left(h_{z}\right) / m_{x}\left(h_{x}\right)=\sqrt{H /\left(H+M_{s}\right)}=1 / \varepsilon<1$, where $m_{x}\left(h_{z}\right)$ and $m_{x}\left(h_{x}\right)$ are contributions of the PP and IP components of the microwave field to $m_{\mathrm{x}}$. From this formula one sees that for the film geometry the in-plane component of the driving field is more important than the PP one. For very small applied fields or large saturation magnetisations for the material $\left(H<<M_{s}\right)$ the contribution of $h_{z}$ becomes even more negligible. Physically this happens because of the large ellipticity of the precession of magnetisation $\varepsilon=m_{x} / m_{z}$ in a film for $H<<M_{s}$. As a result, for ferromagneticmetallic and half-metallic films the in-plane component of the driving field produces the dominating contribution to the stripline FMR response, because typically for these materials $\mu_{0} M_{\mathrm{s}}>1 \mathrm{~T}$ or so. For ferrimagnetic materials, $M_{\mathrm{s}}$ is usually significantly lower. Accordingly, the precession of magnetisation for them is more circular and the PP component of $\mathbf{h}$ plays a much more important role in the formation of the FMR response. (Note that this is also the reason why the non-reciprocity of excitation of MSSW [see Fig. 16(c)] in ferromagnetic yttrium-iron-garnet (YIG) films [89] is much stronger than in ferromagnetic Permalloy films [168, 169]). 
Above we did not assign particular values to the amplitudes $h_{x}$ and $h_{z}$. More specifically, in that analysis we implicitly assumed that $\left|h_{x}\right| \geq\left|h_{z}\right|$. Indeed, as we will show later on with a simple numerical simulation (Sect. 5.2.1, Fig. 24), the two components have usually comparable amplitudes while induced by a microwave current in a stripline.

Experimentally, the contributions of the two microwave field components to the stripline FMR response were studied in [86]. Two samples were used in this study - a $100 \mathrm{~nm}$-thick Permalloy continuous film and an array of parallel nanostripes made from Permalloy and having the same thickness as the continuous film. The width of the individual nanostripes was $264 \mathrm{~nm}$. The BFMR measurements were taken in two configurations: the microstrip line was oriented either parallel (i) to the applied field or perpendicular (ii) to it. The width of the microstrip line was $0.3 \mathrm{~mm}$. The results of these measurements are shown in Fig. 19. One sees that the FMR response taken in Configuration (ii) is smaller than for Configuration (i) for both continuous film and nanostripe array. One also notices that in the case of the continuous film the difference in responses is about $30 \mathrm{~dB}$. However, for the nanostripes the difference in responses is not very significant.

In Configuration (i), both components of the microwave magnetic field can contribute to the FMR response, but only the PP component of the field is able to drive the magnetisation precession in Configuration (ii). Hence, the data taken for the continuous film are in agreement with the prediction of the theoretical analysis above: for the high-magnetic moment ferromagnetic films the impact of the PP component of the microwave magnetic field of the stripline on the BFMR response is negligible. However, this is definitely not the case for the nanostripes. The explanation for this difference in behaviours can be found recalling that the impact of $h_{z}$ scales as $1 / \varepsilon$. The in-plane nanopatterning produces a significant in-plane component of the dynamic demagnetising field of precessing magnetisation. This reduces the ellipticity of the magnetisation precession ( $\varepsilon$ becomes closer to 1). As a result, $h_{z}$ contributes strongly to the amplitude of the dynamic magnetisation.

Below, in Section 7.4, we will use the latter fact to explain the experimental results from that section.

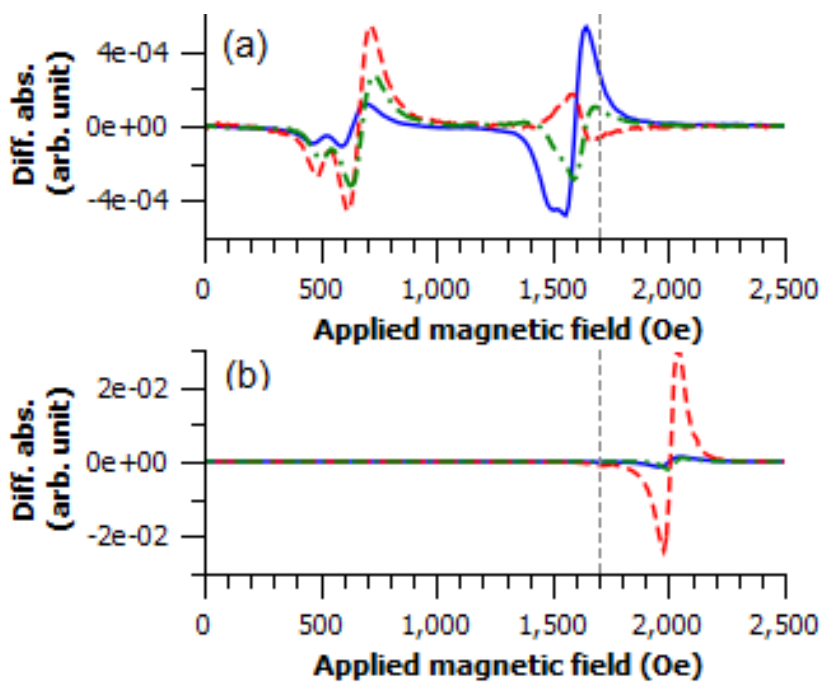

FIG. 19 (a) Nanostripe array, (b) Continuous film. Dashed line - stripline BFMR with the microstrip line parallel to the applied field (and parallel to the nanostripes for (a)). Dash-dotted line - stripline BFMR with the microstrip line perpendicular to the applied field (and to the nanostripe 
array for (a)). For the meaning of the other lines, which are not relevant to the discussion in this review article, see [86]. Taken from [86].

\section{Shielding of the microwave electromagnetic field of striplines by a metal film 5.1 Numerical analysis}

It is evident that in an electrically conducting thin film the shielding by eddy currents occurs independently of the magnetic properties of the film. Indeed, eddy currents are induced inside both magnetic and non-magnetic conducting thin films by a microwave magnetic field incident on a film surface. According to a theory from Ref. [170], at ferromagnetic resonance the shielding effect can be different from the one at off-resonance frequencies. However, the origin of the eddy current shielding will always be due to intrinsic electrical conductivity of the film. Consequently, in a first approximation one can assume that the thin film is non-magnetic but conducting.

Hereafter, we discuss the results obtained by M. Bailleul [171] who used this approach. He employed a rigorous numerical method and an intuitive theoretical model to investigate the impact of the shielding effect on coplanar waveguide broadband FMR measurements. Figure 20(a) shows the geometry used in the simulations by means of a finite element method (FEM). This design mimics the transverse cross-section of a commercial test board fabricated using the conventional printed circuit technique over an RO4003 substrate.

The simulations were carried out using commercial COMSOL Multiphysics software implementing a FEM algorithm. This software solves the Maxwell's equations describing the propagation of electromagnetic waves along an infinite transmission line with a cross-section sketched in Fig. 20(a). An eigenvalue problem for these equations was solved for the microwave frequency of $2 \mathrm{GHz}$ to obtain the only one allowed propagation mode, which is predominantly TEM, and its propagation wave vector $k_{\mathrm{z}}$. The FEM software also allowed simulating the transverse distributions of the electric $\mathbf{E}$ and magnetic $\mathbf{H}$ field, as well as extracting the propagation index $n_{\text {eff }}$ and the characteristic impedance $Z_{\mathrm{c}}$. 

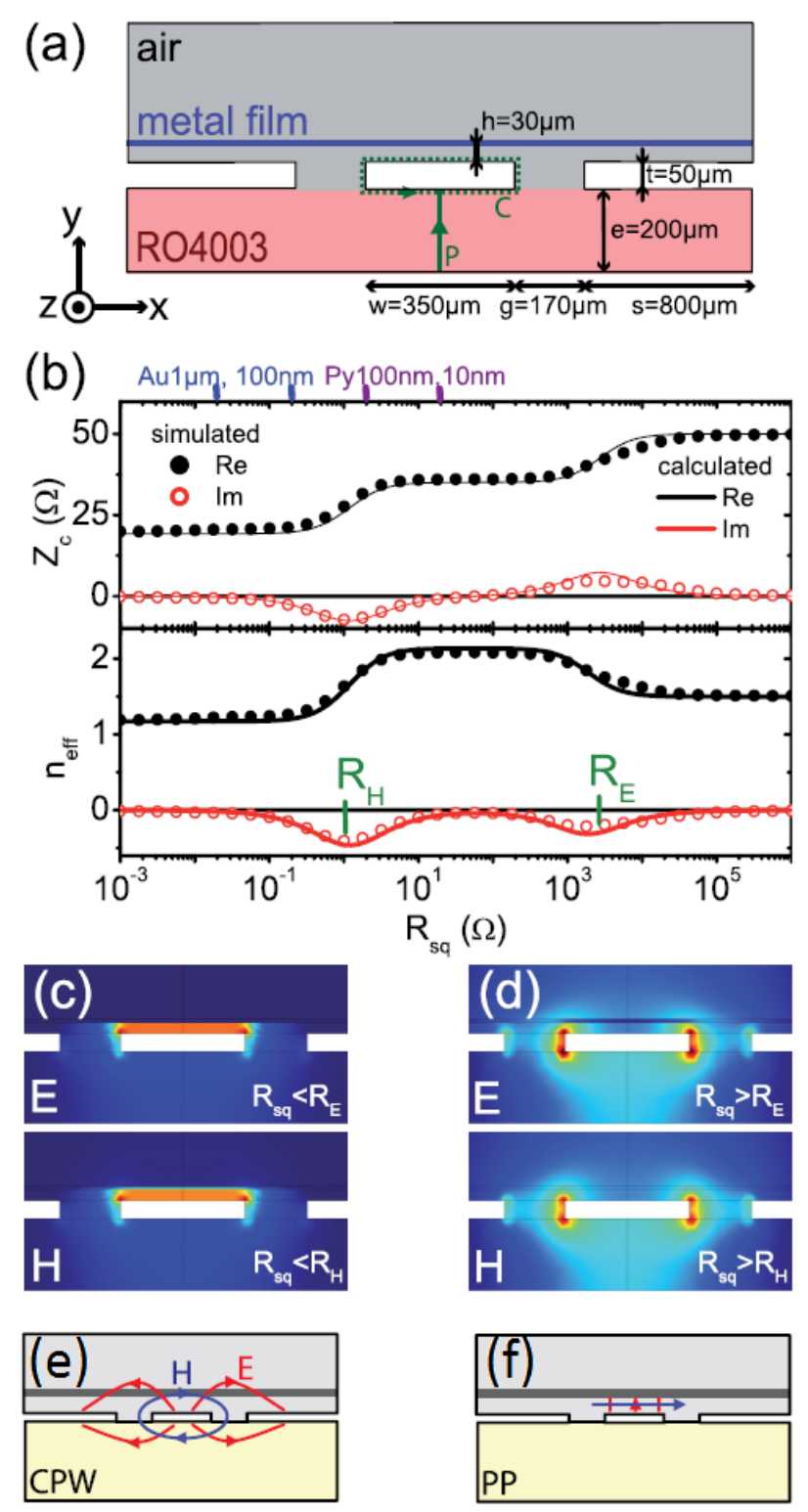

FIG. 20 (a) Geometry of the FEM simulation. (b) Results of the solution of the eigenvalue problem for the microwave frequency $2 \mathrm{GHz}$ (dots). The real and imaginary parts of the characteristic impedance $Z_{\mathrm{c}}$ (top) and the effective propagation index $n_{\text {eff }}$ (bottom) as a function of the resistance per square of the film $R_{\mathrm{sq}}$. The lines are the result of the transmission line model. (c) Amplitude maps of the microwave magnetic and electric fields for $R_{\mathrm{sq}}=1 \Omega$. (d) same for $R_{\mathrm{sq}}=100 \mathrm{k} \Omega$. Sketches of the modes of the coplanar (e) and parallel plate (f) transmission lines. From [171].

Figure 20(b) shows the real and imaginary parts of $n_{\text {eff }}$ and $Z_{\mathrm{c}}$ as a function of the resistance per square of the film $R_{\mathrm{sq}}$, varied between $10^{-3}$ and $10^{6} \Omega$. (Recall that the sheet resistance is a measure of the resistance of thin films that are nominally uniform in thickness.) We distinguish clearly three regions where the curves for the real parts of $n_{\text {eff }}$ and $Z_{\mathrm{c}}$ are nearly flat. These regions are separated by transition regions in which the real parts of $n_{\mathrm{eff}}$ and $Z_{\mathrm{c}}$ vary smoothly but their imaginary parts show peaks denoted as $R_{\mathrm{H}}$ and $R_{\mathrm{E}}$. Different field distributions of $\mathbf{E}$ and $\mathbf{H}$ as well as different values of $n_{\mathrm{eff}}$ and $Z_{\mathrm{c}}$ are observed for each of the three regions. For $R_{\mathrm{sq}}>R_{\mathrm{E}} \approx 2.5 \mathrm{k} \Omega$, both $\mathbf{E}$ and $\mathbf{H}$ are concentrated in the gaps between the signal line and the lateral ground planes 
[Fig. 20(d)] and one also recovers $Z_{\mathrm{C}}=50 \Omega$ and $n_{\text {eff }}=1.5$ targeted in the design of the coplanar line (i.e. there is no effect of the conductivity of the thin film). For $R_{\mathrm{sq}}<R_{\mathrm{H}} \approx 1 \Omega$ (i.e. for a strongly conducting thin film), both $\mathbf{H}$ and $\mathbf{E}$ fields become strongly concentrated in the air gap between the signal line and the metal film [Fig. 20(d)]. In this case, $n_{\text {eff }}$ and $Z_{\mathrm{c}}$ are strongly reduced to 1.2 and $20 \Omega$, respectively. For $R_{\mathrm{H}}<R_{\mathrm{sq}}<R_{\mathrm{E}}$, the distributions of the electric and magnetic fields are different from each other because $\mathbf{E}$ is concentrated above the signal line, but $\mathbf{H}$ is distributed around the signal line. The corresponding values of $n_{\text {eff }}$ and $Z_{\mathrm{c}}$ are 2.1 and $35 \Omega$, respectively.

The physical interpretation of these results is possible within the standard formalism of transmission lines. In the high resistance regime, the effect of the metal film is negligible and one recovers the characteristics of the unperturbed coplanar line [Fig. 20(e)]. In the low resistance limit, the metal film acts as a perfectly conducting ground plane that dominates over the other ground planes because of its proximity to the signal line. Hence, the propagation mode resembles that of a transmission line consisting of two parallel plates with air in between [Fig. 20(f)]. The propagation parameters of this transmission line are easily evaluated as $n_{\mathrm{eff}}{ }^{\mathrm{PP}} \approx 1$ and $Z_{\mathrm{C}}{ }^{\mathrm{PP}} \approx\left(\mu_{0} / \varepsilon_{0}\right)^{1 / 2} h / w=32 \Omega$. In this regime, the metal film acts as a perfect magnetic shield, i.e. it carries currents and charges opposite to those existing in the signal track so that the microwave field above the film is zero.

In accord with these observations M. Bailleul distinguishes two types of electromagnetic shielding for the coplanar lines - the electric one and the magnetic one. The electric shielding takes place for $R_{\mathrm{sq}}<R_{\mathrm{E}}$. It is due to the capacitive coupling of the sample to both signal and ground lines of the coplanar waveguide. Because a microwave voltage is applied between the signal line and the ground lines, this coupling results in a microwave current flowing from the signal line to the ground lines, i.e. in the direction perpendicular to the coplanar. (Essentially, the metallic layer acts a shortcircuiting plug.) It is clear, that the Oersted field induced by this current is along the static applied field and hence cannot contribute to the IP FMR response of a metallic ferromagnetic layer. However, in the geometry of the PP FMR this contribution potentially exists.

In the coplanar line configuration, for $R_{\mathrm{sq}}<R_{\mathrm{H}}$ the magnetic shielding adds to the electric one. This type of shielding is due to inductive coupling of the metallic layer to the signal line of the coplanar waveguide. The inductive coupling results in a microwave current flowing in the layer in the direction opposite to the direction of the current in the signal line, as we already stated above. The origin of the current in the metallic layer is the same as in the case of the incidence of a plane electromagnetic wave on a metal surface - an eddy current is induced in the material. The direction of the eddy current is such that the total microwave magnetic field is enhanced in front of the sample (i.e strong back-reflection of the electromagnetic wave takes place) and gradually drops inside the metal with a distance from its surface (skin effect). For more details, see Fig. 21 and its discussion in the main text.

As discussed in [172], the electric shielding is a peculiarity of the coplanar geometry; for the microstrip geometry it is not relevant. This is because of a significant separation of the sample from the microstrip ground plane. The microstrip equivalent of the $s+g$ parameter for coplanar lines [171] is the thickness of the microstrip substrate. The substrate thickness is usually large which leads to a negligible contribution of the electric shielding to the total shielding effect. This was confirmed in [172] by rigorous finite-difference time-domain (FDTD) simulations [173]. The simulations showed that the microwave current in the sample sitting on top of a microstrip line flows in the opposite direction to the one in the microstrip. As follows from the discussion above, this is a clear indication of the dominance of the magnetic shielding regime for microstrip lines.

An approximate analytical model of the electric shielding for the microstrip geometry was 
recently suggested in the appendix to Ref. [161]. This calculation confirmed that the electric shielding is usually negligible for the microstrip geometry.

\subsection{Intuitive analytical approach}

In this Section, we reproduce a simple analytic electrodynamic theory of magnetic shielding from Appendix to [90] and from [174]. An example of a microstrip line is used, because it is easier to analyse. Qualitatively, a similar behaviour is expected for the coplanar-line-based BFMR, which was confirmed by the numerical work by M. Bailleul [171], which we discussed in the preceding section.

Before we start, it is instructive to give more details of the structure of the electromagnetic field of a microstrip line. In the direction perpendicular to the microstrip surface the electromagnetic field is evanescent in nature [Fig. 6(a)]. It is (quasi)-transverse (TE) with respect to the direction of propagation of the microwave signal (along the microstrip). The transverse electric field of a typical microstrip line used in BFMR measurements is essentially electrostatic in nature. The distribution of this field resembles the one of a capacitor formed by the microstrip and the ground plane [91]. In the space above the microstrip this field represents a stray field of a non-ideal capacitor; this evanescent field does not vanish because of a finite width of the microstrip. The evanescent dynamic magnetic field of the microstrip line represents a superposition of the Oersted fields of the current in the microstrip and of the return (mirror) current in the ground plane. If the Ohmic resistance of the microstrip is negligible, in the quasi-static approximation [91] the magnetic and electric fields are independent of each other. However, the magnetic field is dual to the electric field [175], which results in a specific value of the characteristic impedance for a microstrip line. As the width of the microstrip determines the structure of the fields, calculation of microstrip dynamic fields taking into account displacement currents is an intrinsically two-dimensional (2D) problem, which is difficult to solve in a general case [176].

To circumvent this problem and to get a valid insight into the structure of the electromagnetic fields of a stripline loaded with a conducting film, in Ref. [90] it was suggested to consider a number of simplified 1D layered geometries which mimic a stripline [Fig. 22(a, b)]. In this analysis, the ferromagnetism of the film is neglected and the film is considered as metallic nonmagnetic. Two of the considered geometries [B and C in Fig. 22(a, b)] - are also characterised by evanescent fields and therefore represent more appropriate models than $\mathrm{A}$, which is the case of a transverse electromagnetic wave incident on a metallic magnetic film. However, below we will concentrate on the model A, because it results in simple and explicit analytical equations.

In the geometry $\mathrm{A}$, the incident electromagnetic field is not evanescent in nature. It represents a plane electromagnetic wave incident normally onto the surface of a single-layer metallic non-magnetic film. This simple model is adequate to our purposes because, as shown experimentally in Ref. [177], ferromagnetic metallic films demonstrate the same behaviour in the conditions of the plane electromagnetic wave transmission/reflection experiment and the conditions of the stripline BFMR setup.

The situation of a transverse electromagnetic wave incident on a metallic magnetic film can be analysed using an approach from the field of infra-red optics. This is because the properties of thin non-magnetic conducting films with thicknesses smaller than the classical skin depth for infrared applications were extensively discussed in the past [178, 179] (see also [180]). However, whereas in optics the attention was not paid to the distribution of the electromagnetic field across the film thickness, this distribution is of immediate relevance to the topics of this review article and 
it will be discussed below.

The system of equations which follows from the Maxwell's equations for the geometry A is simple and is given by Eqs. (B1) in Ref. [90]. The solution of this system of equations is shown in Fig. 21(a). One sees that most of the electric-field component of the wave is reflected from the front film surface, but this surface is fully transparent to the magnetic component of the electromagnetic field. The microwave magnetic field penetrates through this surface and forms a very peculiar asymmetric and highly non-uniform field profile inside the film. The strong profile asymmetry is due to almost total internal reflection of the magnetic field from the far film surface. Because of a very large difference in the microwave refractive indices for the metal ( $\sqrt{\varepsilon}=\sqrt{\sigma /\left(i \omega \varepsilon_{0}\right)}$ ) and the space surrounding the film (air) the phase of field reflection is 180 degree, which results in destructive interference of the forward-propagating and reflected wave at the far interface. The (almost) total reflection implies that the amplitudes of the incident and the reflected wave are (almost) the same. This results in an (almost) vanishing amplitude of the total microwave magnetic field at the far film surface.

The linear profile of the standing wave inside the metallic film of a thickness $L$

$$
h_{\mathrm{x}}(y)=2 \frac{L-y}{L},
$$

seen in the right panel to Fig. 21(a) represents the interference pattern of the two waves. The wave number $\omega /\left(\sqrt{\varepsilon} c_{0}\right)$ for this wave is complex because the dielectric permittivity $\varepsilon$ for metals is a complex value. (Here, $\omega$ is the microwave angular frequency and $c_{0}$ is the speed of light in vacuum.) Therefore, given the node at the far film surface, the interference pattern is essentially a hyperbolic sine function whose Taylor expansion for $k y<<1$ is given by Eq. (47).

(a)

(b)

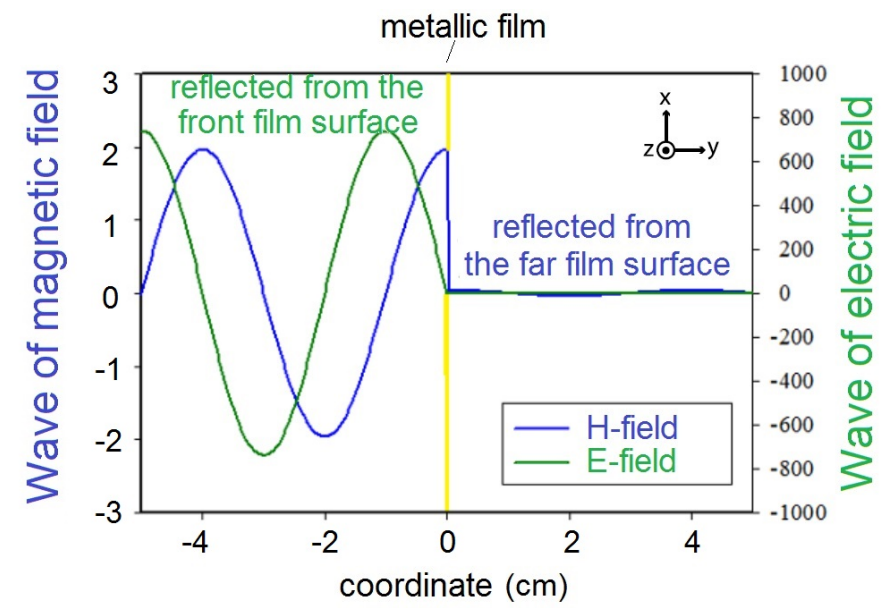

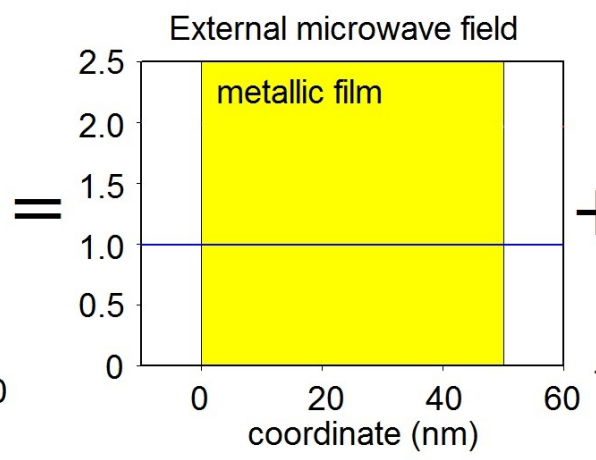

60
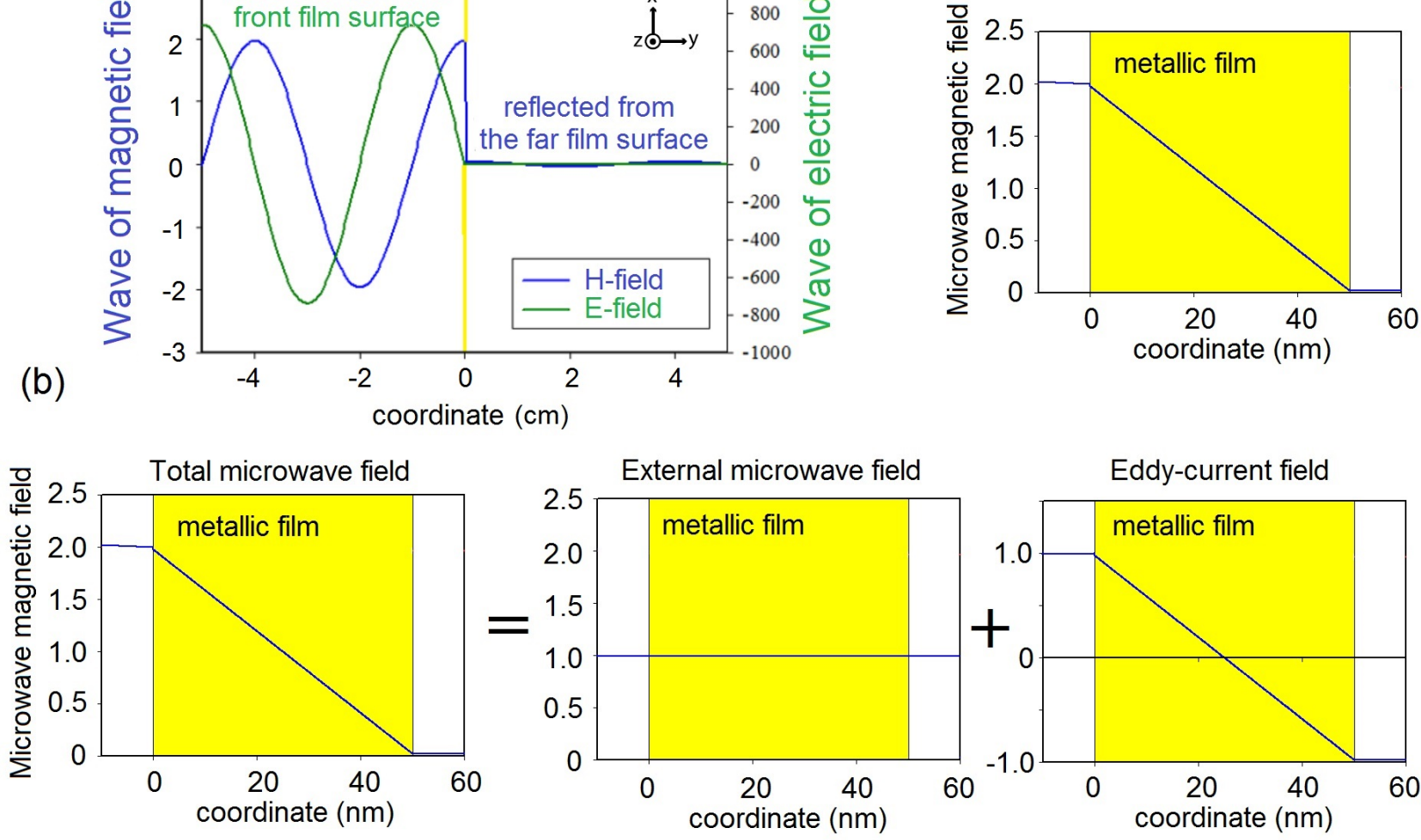
FIG. 21 (a) Left panel: Simulated electric field wave (green line) and magnetic field wave (blue line) of an electromagnetic wave normally incident on a sub-skin-depth-thick metallic film. Right panel: Close-up of the microwave magnetic field wave inside the metallic film. Note the difference between the units of the $x$-axes of the left and right panels. The microwave frequency is $8 \mathrm{GHz}$. (b) Explanation of the formation of the profile of the microwave magnetic field wave inside the film.

Alternatively, the formation of the field profile in the inset to Fig. 21(a) may be thought as a combination of the two profiles in Fig. 21(b): the uniform one of the microwave field of the incident electromagnetic wave (perfectly uniform on the length scale of the film thickness) and of the perfectly anti-symmetric Oersted field of the perfectly uniform eddy current flowing in the film.

The result in Eq. (47) is valid for the conductivity of metals and for thin films of sub-skindepth thickness, since only for them the eddy current density is uniform (the eddy current density scales as the first derivative of Eq. (47) with respect to $y$ ). For thicker films $(\operatorname{Im}\{k L\}>1)$ the usual skin effect is recovered, since the forward propagating wave cannot reach the far film surface and thus no back reflection from that surface is present.

Let us now find out for which set of material parameters Eq. (47) is valid. For this purpose let us assume that the amplitude of the microwave magnetic field at the front film surface is 1 , and that $|k L|$ is always less than one, which implies that we are interested only in the films with subskin-depth thicknesses. Then $h_{\mathrm{x}}(y)$ is always a linear function. Therefore, the amplitude of the microwave magnetic field at the far film surface $A_{\text {far }}$ ("transmitted field") can be regarded as a measure of the asymmetry of the $h(x)$ profile.

It has been shown $[90,179]$ that $A_{\text {rear }}$ depends on the thickness and conductivity of the film as follows

$$
A_{\text {rear }}=\frac{1}{1+\frac{Z_{0}}{\rho} \frac{L}{2}},
$$

where $\rho=1 / \sigma$ is the electrical resistance of the film, $Z_{0}=377 \Omega$ is the characteristic impedance of free space. In Fig. 22(c), which was calculated for the standard conductivity of Permalloy $\left(\sigma=4.5 \times 10^{6} \mathrm{~S} / \mathrm{m}\right)$, one sees that for $L=25 \mathrm{~nm}$ the amplitude of the microwave field at the rear boundary is $A_{\text {rear }}=0.04$. Thus, starting from this minimum film thickness the shielding is nearly perfect. (For comparison, the classical skin depth for a non-magnetic metal with the conductivity of Permalloy is $1.2 \mu \mathrm{m})$. Remarkably, the frequency term does not enter Eq. (48). Importantly, as follows from Fig. 22(c), even for a $100 \mathrm{~nm}$ thick film the law Eq. (47) and the boundary condition

$$
h_{\mathrm{x}}(y=L)=0
$$

which follows from Eq. (48) for $\rho / L<<Z_{0}$ are valid approximations.

To include ferromagnetism into the discussion one might neglect the exchange interaction and introduce an effective relative scalar microwave permeability using the Almeida-Mills formula [170]

$$
\mu_{\mathrm{v}}=\frac{\mu^{2}-\mu_{\mathrm{a}}^{2}}{\mu},
$$

where $\mu_{\mathrm{v}}$ and $\mu_{\mathrm{a}}$ are the diagonal and off-diagonal components of the microwave magnetic 
permeability tensor, respectively. However, it can be shown that in this case Eq. (48) remains unchanged because $\mu_{\mathrm{v}}$ cancels out in the final expression.

The characteristic impedance of microstrip lines is usually $50 \Omega$, which is much smaller than the characteristic impedance of vacuum. If one replaces $Z_{0}=377 \Omega$ with $Z_{0}=50 \Omega$ in Eq.(4.2) one obtains a larger transmission as shown in Fig. 22(c) by a thin solid line. However, it remains considerably smaller than that follows from the magnetic skin depth law $h_{\mathrm{x}}(L) / h_{\mathrm{x}}(0)=\exp (-\delta / L)$ [dotted line in Fig. 22(c)], where $h_{\mathrm{x}}$ is the in-plane component of the dynamic magnetic field [90].

(a)

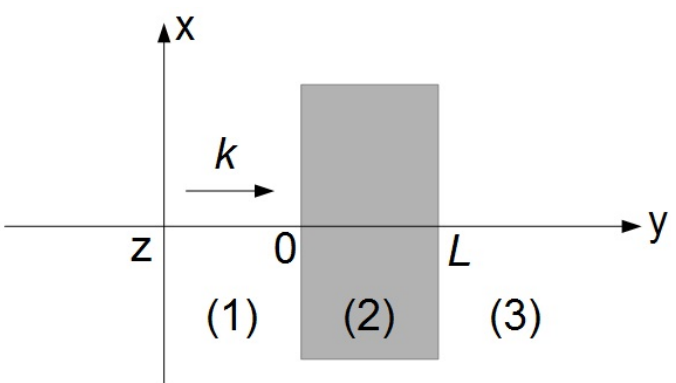

(b)

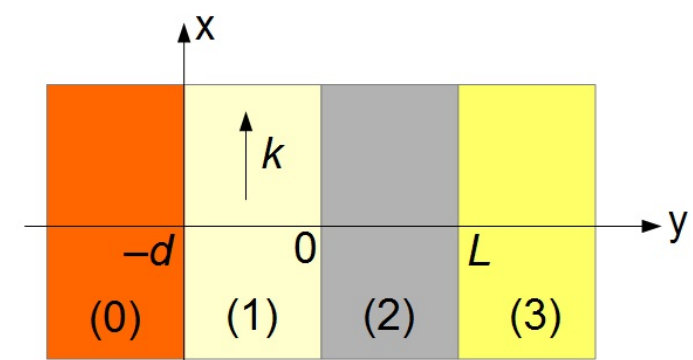

(c)

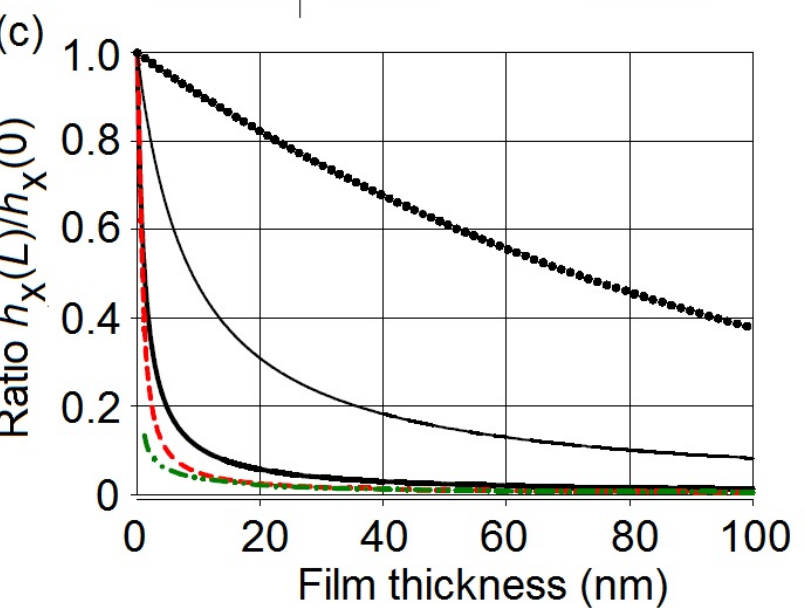

FIG. 22 (a) Geometry of case A. The areas (1) and (3) are dielectric half-spaces, and the area (2) is a metallic magnetic layer. (b) Geometry of cases B and C. Case B: the area (0) is an area with a characteristic impedance of $50 \Omega$ in which a TM wave propagates along the $x$-axis. This wave is given by a microwave current at the surface $y=-d$. (1) and (3) are insulating layers and (2) is a magnetic metallic layer. Case C: (1) and (3) are insulating layers, (2) is a metallic magnetic layer, and the space $y<-d$ is an ideal conductor. (c) Ratio of the amplitude of the dynamic magnetic field at the rear surface of the film to its value at the front surface of the film $h_{\mathrm{x}}(L) / h_{\mathrm{x}}(0)$. The conductivity of the film is $4.5 \times 10^{6} \mathrm{~S} / \mathrm{m}$, the effective scalar microwave permeability $\mu_{\mathrm{v}}=i 360$ at the driving microwave frequency $7.5 \mathrm{GHz}$. Black thick solid line - the case of a transverse electromagnetic wave incident on a metallic magnetic film; the film is surrounded by two layers with the dielectric permittivity $\varepsilon=1$. Black thin solid line - the same magnetic film, but the characteristic impedance of the surrounding space is set to $50 \Omega$. Red dashed line - multilayer 
structure driven by a wave of current over an ideally conducting surface. Green dash-dotted line dielectric waveguide with one surface metallised. Dotted line - the magnetic skin depth law. From [90].

In the framework of another "equivalent layered structure" - a multilayer structure driven by a wave of current over an ideally conducting surface - one sees a similar trend as in the case of the excitation with a plane wave [Fig. 22(c)]. For magnetic films which are thicker than $30 \mathrm{~nm}$ the microwave magnetic field behind the film has a negligibly small amplitude. Furthermore, the profile of the dynamic magnetic field inside the ferromagnetic film is given by the same equation, Eq. (47).

Finally, in the case of the dielectric waveguide with one surface metallised one needs to considerably increase the thickness of the insulating layer between the metallisation and the ferromagnetic film in order to ensure the guided-wave propagation. The typical suitable thickness is $d=3.5 \mathrm{~mm}$ and larger. The results in Fig. 22(c) are for $d=3.5 \mathrm{~mm}$, which results in the characteristic impedance $58 \Omega$ for the film thickness $L=100 \mathrm{~nm}$. One sees that in this case the Oersted field of eddy currents shows the same strong inhomogeneity across the film thickness as in the previous examples, which model to some extend a thin metallic film on top of a wide stripline.

From the discussion above one sees that an efficient shielding of the microwave magnetic field is not a property of some particular geometry, but is rather typical in the cases when the dynamic magnetic field is applied only from one side of the film. The difference between the electrical resistance of the film and the characteristic impedance of neighbouring layers is important. If this difference is large, an efficient shielding occurs [see Eq. (48)]. In these conditions the dynamic magnetic field easily penetrates inside the film from the side of field concentration. However, it undergoes almost a total reflection back into the film at the second film surface. Importantly, the presence of another conducting layer [in the cases B and C in Fig. 22] and thus the capacitance between the film and the conducting layer do not modify this effect.

Finally, a short comment on the applicability of Eqs. (47-49) is appropriate. Above we noted that frequency did not enter Eq. (48). This means that this shielding works for any field frequency, excluding the absolute zero frequency. This is in contradiction with the common sense, as the latter suggests a gradual decrease in this effect with a decrease in the frequency. However, this result just evidences that some important parameter potentially takes a limiting value of zero or infinity in the model. This parameter is the length of the ferromagnetic film in the direction $z$ of the microwave current. The model implicitly assumes that the sample length in this direction is infinite. This assumption ensures the continuity of the eddy current in the film. The electromagnetic induction law tells us that eddy currents will exist in a material for any non-zero frequency as long as the eddy-current lines are continuous. For the present geometry this leads to the requirement of an infinite length of the sample in the $z$ direction.

This observation allows one to conclude that this model is valid for real geometries for which the eddy current loops can be closed without inserting considerable extra impedance. In real films of finite lengths along the $z$ direction, the continuity of the eddy current may be ensured due to the capacitance between the two opposite film edges which are along $x$ and/or between the film and the signal line of the coplanar line or the microstrip of the microstrip line. In both cases the extra impedance in the form of capacitive reactance increases with a decrease in the frequency. Thus, one cannot expect a noticeable shielding at frequencies below the microwave range. Indeed, from literature on electromagnetic shielding $[181,182]$, one finds that at small frequencies closed (e.g., 
spherical) shielding enclosures are necessary to ensure the continuity of eddy currents.

\subsubsection{Inclusion of the finite width of the stripline transducer}

So far, we discussed results obtained using a simple analytical approach, which assumes that the stripline transducer has an infinite width in the sample plane. However, real striplines have finite widths. This may have implications to their operation as BFMR transducers. One of these implications has already been discussed in Section 4 where it has been shown that the finite transducer width may lead to travelling spin wave contribution to the resonance line width. In this section, we show that the finite transducer width may also modify the microwave magnetic shielding effect.

Similarly to the preceding two sections, to keep the analysis simple, we treat the metallic film as non-magnetic. This analysis follows the theory from [183]. It uses an approach in the Fourier space because this approach allows one to obtain simple analytical formulas.

For the stripline Fig. 6 and a non-magnetic metallic film from the Maxwell's equations one obtains

$$
\begin{gathered}
i k h_{y k}+\frac{\partial h_{x k}}{\partial y}=-\sigma e_{z k} \\
\frac{\partial e_{z k}}{\partial y}=-i \omega \mu_{0} h_{x k} \\
-i k h_{x k}+\frac{\partial h_{y k}}{\partial y}=0
\end{gathered}
$$

where $\omega /(2 \pi)$ is the driving microwave frequency, $\sigma$ is the conductivity of the film, and all components of the microwave fields $\mathbf{h}$ and $\mathbf{e}$ are represented as Fourier expansions in the in-plane direction $x$ as

$$
\mathbf{h}, \mathbf{e}=\int_{-\infty}^{\infty} \mathbf{h}_{k}, \mathbf{e}_{k} \exp (i \omega t) \exp (-i k x) d x
$$

By differentiating Eq. (51) and combining it with Eqs. $(52,53)$ one obtains

$$
\begin{aligned}
& \frac{\partial^{2} h_{x k}}{\partial y^{2}}-K^{2} h_{x k}=0, \\
& h_{y k}-\frac{i k}{K^{2}} \frac{\partial h_{x k}}{\partial y}=0,
\end{aligned}
$$

where $K^{2}=i \sigma \omega \mu_{0}+k^{2}$ inside the film.

Outside the film $\sigma=0$ and $K^{2}$ reduces to $k^{2}$. We require that the magnetic field vanishes in the regions far behind and in front of the film where $\sigma=0$. Then, from the conditions of continuity of $h_{\mathrm{x}}$ and $e_{\mathrm{z}}$ at the surfaces of the film one obtains the following conditions at the surfaces of the film from inside the film: 


$$
\begin{aligned}
& h_{x k}+i h_{y k} \frac{k}{|k|}=0, \quad \text { for } \quad y=L \\
& h_{y k}-i h_{x k} \frac{k}{|k|}=j_{k} \frac{k}{|k|}, \text { for } y=0
\end{aligned}
$$

where $j_{k}$ is the linear current density for a current sheet of width $w$ and infinitely small thickness which mimics the stripline. The sheet is located at $y=0$ which is right on the film surface, i.e. no spacer between the stripline and the film is assumed in the calculation.

The solution of Eq. (55) satisfying the boundary conditions Eq. (57) reads:

$$
\begin{aligned}
& h_{x k}=K \frac{K \sinh [K(y-L)]-|k| \cosh [K(y-L)]}{\left(K^{2}+k^{2}\right) \sinh (K L)+2 k K \cosh (K L)} j_{k} \\
& h_{y k}=i k \frac{K \cosh [K(y-L)]-|k| \sinh [K(y-L)]}{\left(K^{2}+k^{2}\right) \sinh (K L)+2 k K \cosh (K L)} j_{k} .
\end{aligned}
$$

where $K$ is the principal square root of the complex value $i \sigma \omega \mu_{0}+k^{2}$.

Figure 23 demonstrates a result of the calculation using the formulae Eq. (58). From Fig. 23 one sees that for $k=0$ the microwave shielding is perfect, but it drops quickly with an increase in $k$. Recall that the spectrum of spatial Fourier amplitudes of a stripline geometry is located in the range $|k|<2 \pi / w$, where $w$ is the characteristic width of the stripline. Thus, from Fig. 23 one may expect that the shielding efficiency should decrease with a decrease in $w$. Indeed, this is confirmed by the numerical inverse Fourier transform of Eq. (58) with $j_{\mathrm{k}}$ in the form which corresponds to a uniform distribution of the current density over the stripline width: $j_{k}=\frac{w}{2 \pi} \frac{\sin (k w / 2)}{k w / 2}$. Figure 24 shows two examples of this calculation. One of them is for $w=300 \mu \mathrm{m}$, which corresponds to the width of the signal line of the coplanar BFMR transducer investigated numerically by $\mathrm{M}$. Bailleul (Section 5.1), and also to the width of a microstrip line largely used in our experiments [86, 184]. From Fig. 24 one sees that the eddy current density is uniform across the film thickness for both values of $w$. For $w=300 \mu \mathrm{m}$ the eddy currents leads to an almost perfect shielding effect: The amplitude of both field components is small at the far film surface $(y=L)$. Furthermore, $h_{\mathrm{x}}(y=0) / j$ is close to 1 . This is very similar to $h_{\mathrm{xk}}(y=0) / j_{\mathrm{k}}=1$ for $k=0$ or, equivalently for $w=\infty$, given by the first equation of Eqs. (58). For $w=30 \mu \mathrm{m}$, both $h_{\mathrm{x}}(y=0) / j$ and $h_{\mathrm{x}}(y=L) / j$ are close to 0.5 . The latter value is characteristic of an insulating film with $L<<w$.

Thus, a $300 \mu \mathrm{m}$-wide stripline may be considered as one which produces a strong shielding effect, but the $30 \mu \mathrm{m}$-wide one is essentially microwave-magnetic-shielding-free. This conclusion is in good agreement with experiment. Experimentally, one sees strong evidence of the shielding contribution to FMR amplitudes for $300 \mu \mathrm{m}$-wide microstrip and coplanar transducers. But no evidence of this effect has been observed so far in the experiments on excitation of travelling spin waves in metallic ferromagnetic films with stripline transducers with $w<<10 \mu \mathrm{m}$ (see, e.g., [185]).

As a final remark to this section, we note an analytical exchange-free theory presented in the appendix to Ref. [88]. Being similar to Eqs. (51-58) above, this theory additionally takes into account the ferromagnetism of metallic films. We stress that results produced by this theory are in full agreement with the simple analysis above. For instance, it shows that for a typical coplanar stripline transducer with $w=1.1 \mathrm{~mm}$ [whose Fourier spectrum is shown in Fig. 25(a)] the shielding effect should be strong, as seen from Fig. 25(b). This is in full agreement with our experimental 
data (Fig. 3 in Ref. [88]).

The results above have been also confirmed by recent 2D numerical simulations for singlelayer ferromagnetic films [161]. Both dipole-dipole and exchange interactions were included into the calculation. These simulations showed that for $L=50$ and $100 \mathrm{~nm}$, the FMR response at $15 \mathrm{GHz}$ for $w=350$ and $1500 \mu \mathrm{m}$ is the same as for $w=\infty$, but below $w=100 \mu \mathrm{m}$ the eddy-current contribution to the FMR amplitudes gradually disappears.
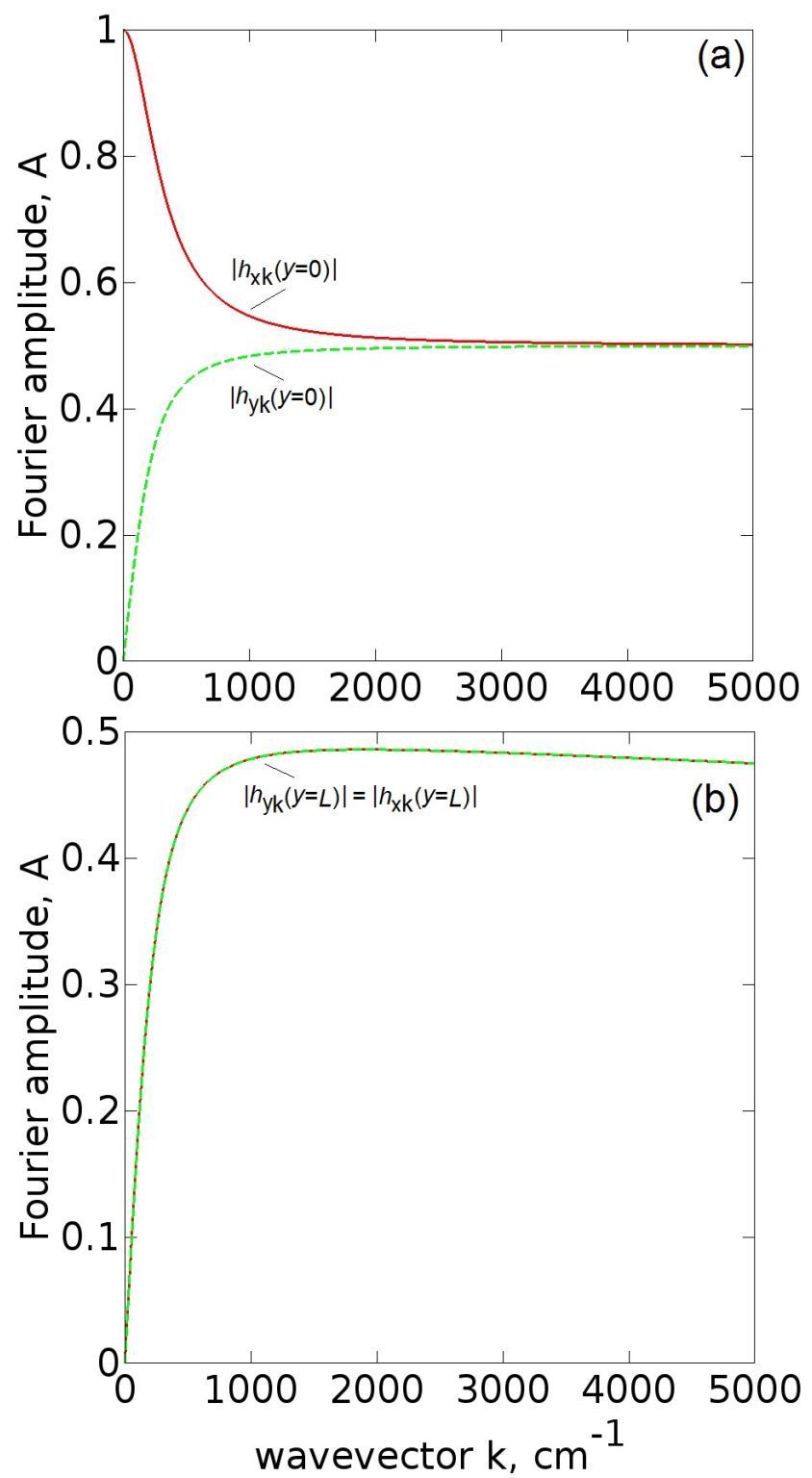

FIG. 23 Profiles of the $h_{x k}$ and $h_{y k}$ - Fourier components of the microwave magnetic field as a function of the Fourier wave vector $k$ at the surfaces of the film: (a) $y=0$ (i.e. at the surface of the film which faces the microstrip line) and (b) $y=L$ (i.e. at the film surface which faces away from the microstrip line). Note that in (b) $\left|h_{y k}\right|=\left|h_{x k}\right|$ for all $k$-values. The microwave frequency is 15 GHz. From [183]. 

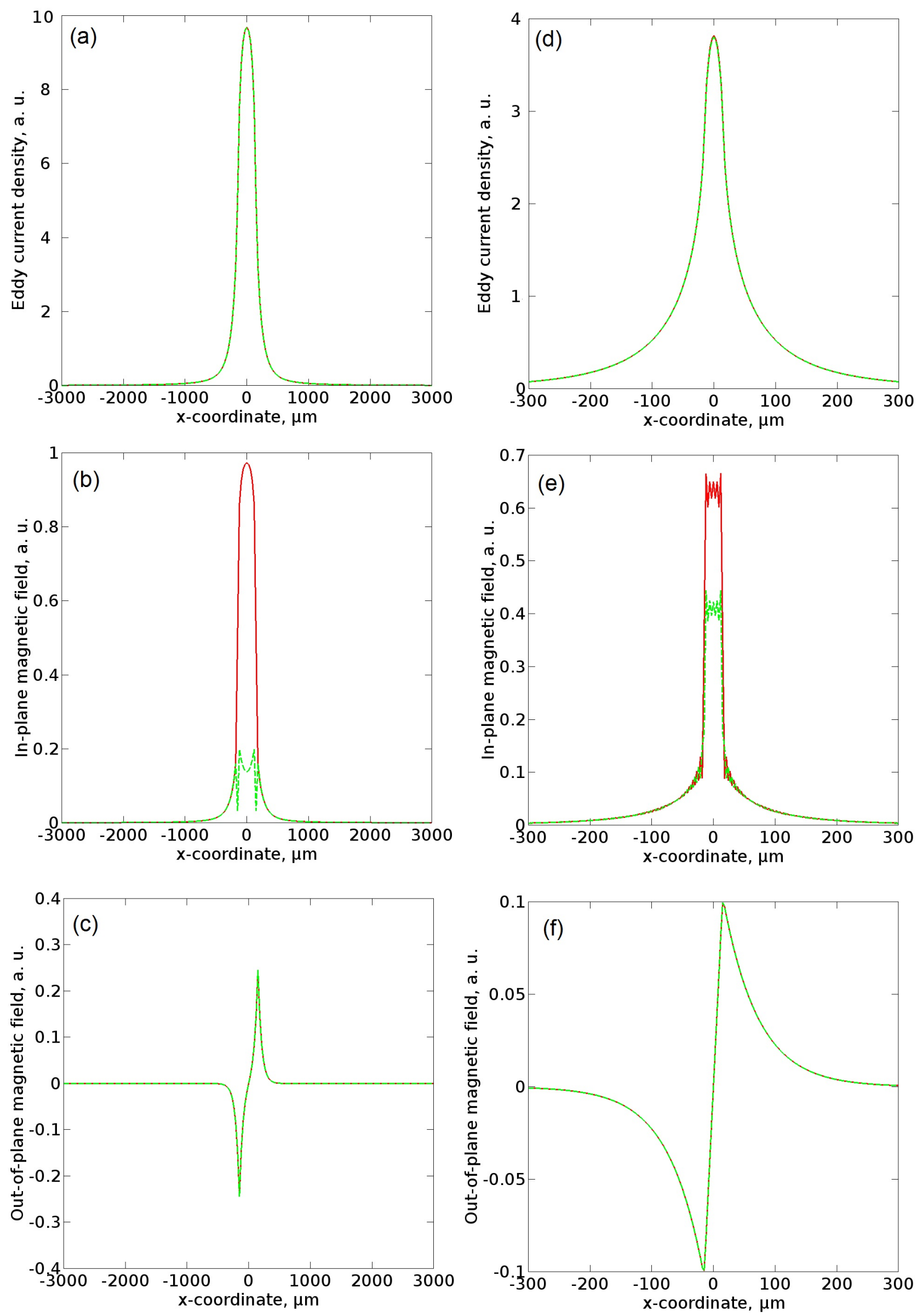

FIG. 24. (a, d) Eddy current density, (b, e) in-plane components $\left(h_{\mathrm{x}}\right)$ of the microwave magnetic field, and (c, f) perpendicular-to-plane components $\left(h_{\mathrm{y}}\right)$ of the microwave magnetic field as a 
function of the $x$-coordinate (the coordinate along the in - plane direction in Fig. 6). (a), (b) and (c): Microstrip width is $300 \mu \mathrm{m}$. (d), (e) and (f): Microstrip width is $30 \mu \mathrm{m}$. The fields are normalised to the microwave current density in the microstrip. Solid lines - field amplitude at the front film surface $(y=0)$. Dashed lines - field amplitude at the far film surface $(y=L)$. Note that the solid and dashed lines coincide to graphical accuracy in the panels (d) and (f). The microwave frequency is $10 \mathrm{GHz}$
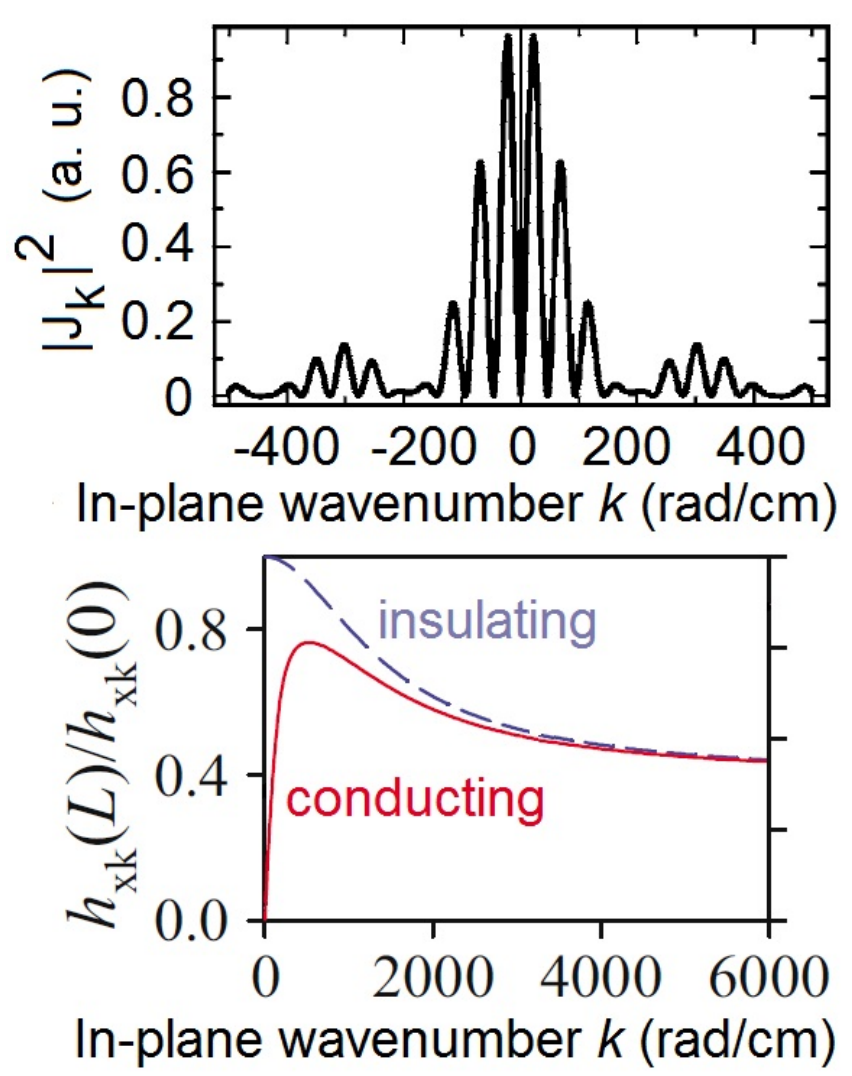

FIG. 25 (a) Spatial Fourier transform of the microwave current density of a coplanar line. The width of the central conductor (the signal line) is $w=1.1 \mathrm{~mm}$ and the slot width is $\Delta=0.24 \mathrm{~mm}$. From [94] (b) Red solid line - relative amplitude of the in-plane Fourier-component of the microwave magnetic field $h_{\mathrm{xk}}(y=L) / h_{\mathrm{xk}}(y=0)$ at the far film surface $y=L$ for a conducting film as a function of the in-plane Fourier wave vector $k$. Blue dashed line - the same but for an insulating film. From [88].

\subsection{Experimental evidence of strong contribution of microwave shielding effect to magnetisation dynamics}

Importantly, the results in Fig. 21(b) and 24(b) are characterised by lack of inversion symmetry in the $y$-direction. This lack of the symmetry is due to the incidence (or concentration) of electromagnetic fields from (at) one side of the magnetic film. Another important aspect is that the BFMR spectroscopy setup is known to excite and detect not only the FMR mode, but also the higher-order SSWMs [96].

From the first of these observations it follows that if there is any impact of the eddy currents and microwave shielding on the magnetisation dynamics of metallic ferromagnetic films, it should be best seen for materials lacking inversion symmetry.

Below we review the results of three separate measurements whose combination delivers 
unambiguous experimental evidence of the eddy-current contribution to the magnetisation dynamics. Historically, these experiments were reported in a sequence different from the one in which we present them below. We believe that the order, in which we discuss them here, is more appropriate from the pedagogical point of view. In all three experiments, materials lacking inversion symmetry were used.

\subsubsection{Transmission/reflection experiment}

The first experiment we would like to mention is from Ref. [177]. In this work, transmission of a microwave electromagnetic wave through a rectangular microwave waveguide filled with a metallic magnetic bi-layer film was investigated [Fig. 26(a)]. A number of exchange-coupled CoPermalloy (Py) films were grown using rf magnetron sputtering. The layer ordering was as follows: first a $5 \mathrm{~nm}$-thick Ta seed layer was grown on a $0.9 \mathrm{~mm}$-thick $\mathrm{Si}$ substrate. Then a thick (50 to $70 \mathrm{~nm}$ ) Permalloy layer was grown on top of it. It was covered by a $20 \mathrm{~nm}$-thick Co layer and the whole stack was capped by $5 \mathrm{~nm}$ of Ta. Important for the experiment was a large area of the substrate $-10 \times 22 \mathrm{~mm}^{2}$; it was needed to tightly fit the cross-section of the waveguide fixture with the sample. 


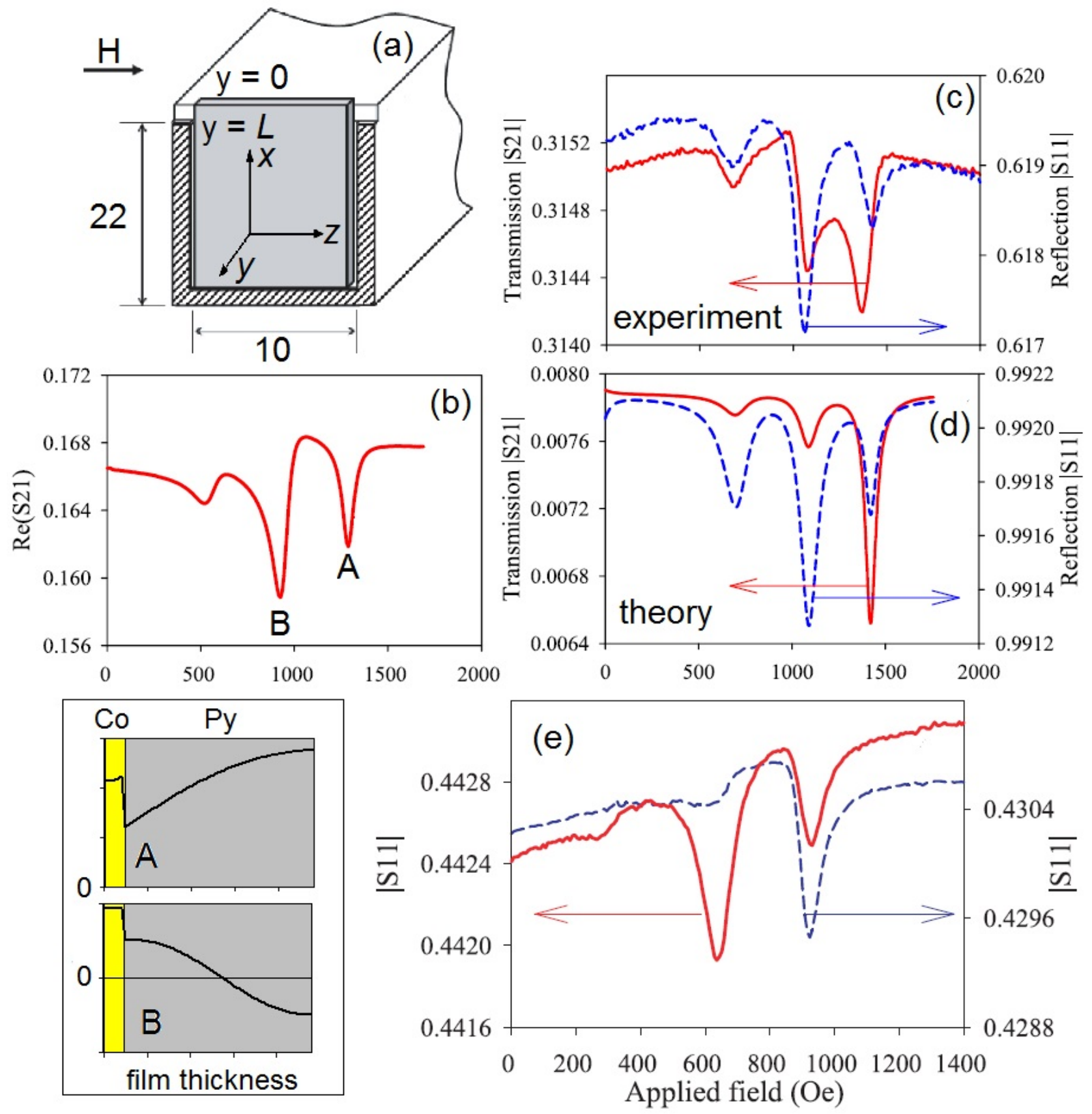

FIG. 26 (a) Sketch of the cross-section of the microwave rectangular waveguide with a magnetic sample (grey parallelepiped). The dimensions of the sample are $10 \times 22 \mathrm{~mm}^{2}$. The wave in the waveguide propagates along the $y$-axis, which is perpendicular to the plane of the sample. The same frame of reference was used in the theoretical work Ref. [174]. (b) Microwave magnetic absorption measured with the stripline BFMR on $\mathrm{Ta}[5 \mathrm{~nm}] / \mathrm{Co}[20 \mathrm{~nm}] / \mathrm{Py}[70 \mathrm{~nm}] / \mathrm{Ta}[5 \mathrm{~nm}] / \mathrm{Si}$ multilayer at the microwave frequency of $10.6 \mathrm{GHz}$. The inset below the Panel (b) sketches the theoretical profiles of the fundamental mode (A) and the $1^{\text {st }} \mathrm{SSW}$ mode (B) across the film thickness. Experimental (c) and theoretical (d) data for the transmission (solid line) and reflection (dashed line) in the waveguide experiment. The film faces the microwave flux. The microwave frequency is 11.184 GHz. (e) Comparison of waveguide data in reflection. Solid line: film faces the incident flux; dashed line: film substrate faces the incident flux. The microwave frequency is $8.865 \mathrm{GHz}$. From [177].

Figures 26(c, d) show the transmission $\left|S_{21}\right|$ and reflection $\left|S_{11}\right|$ characteristics of the 
waveguide fixture with an inserted magnetic sample. The film faces the incident flux. Panel (c) shows the experimental result and Panel (d) shows the theoretical prediction. The measurements were taken in the applied field-resolved configuration and at a given frequency. Panel (e) demonstrates similar experimental data in reflection, but for two sample orientations: with the film facing the incident microwave flux and the film substrate facing the flux. In all experimental data one observes three peaks: the fundamental mode - the upper-field resonance corresponding to the quasi-uniform precession and the $1^{\text {st }}$ and $2^{\text {nd }}$ SSWMs across the film thickness (the middle and the lower-field resonance, respectively). In Fig. 26, the inset below the Panel (b) sketches the theoretical profiles of the fundamental mode [A in Fig. 26(b)] and the $1^{\text {st }}$ SSW mode [B in Fig. 26(b)] across the film thickness. Essentially, these profiles demonstrate that the resonant dynamics is localised in the Permalloy layer and the magnetisation precession in the Co layer is forced; it is driven by the exchange coupling through the interface of the two layers.

Several important observations follow from Figs. 26(c-e):

- The shapes of the traces taken in transmission and reflection are very different;

- The shape of the response in reflection strongly depends on the film orientation, but the shape of the trace taken in transmission is not;

- When the film (essentially the Co layer, since the electrical conductivity of Ta is low as compared with that of Co) faces the incident flux, the amplitude the $1^{\text {st }}$ SSWM measured in reflection is extremely strong with respect to the response of the fundamental mode. However, when the substrate (i.e. the Permalloy layer) faces the microwave flux, the relative amplitude of the response of the $1^{\text {st }}$ SSWM is significantly smaller;

- The trace taken in reflection [Fig. 26(c)] and with the film facing the incident flux is very similar to the reference stripline BFMR trace [Fig. 26(b)].

\subsubsection{Film-flip FMR of metallic bi-layer materials}

The last conclusion of Section 5.3.1 is important for understanding of the second separate experiment we wish to mention in this section. Fig. 27 shows experimental data from Ref. [88]. 


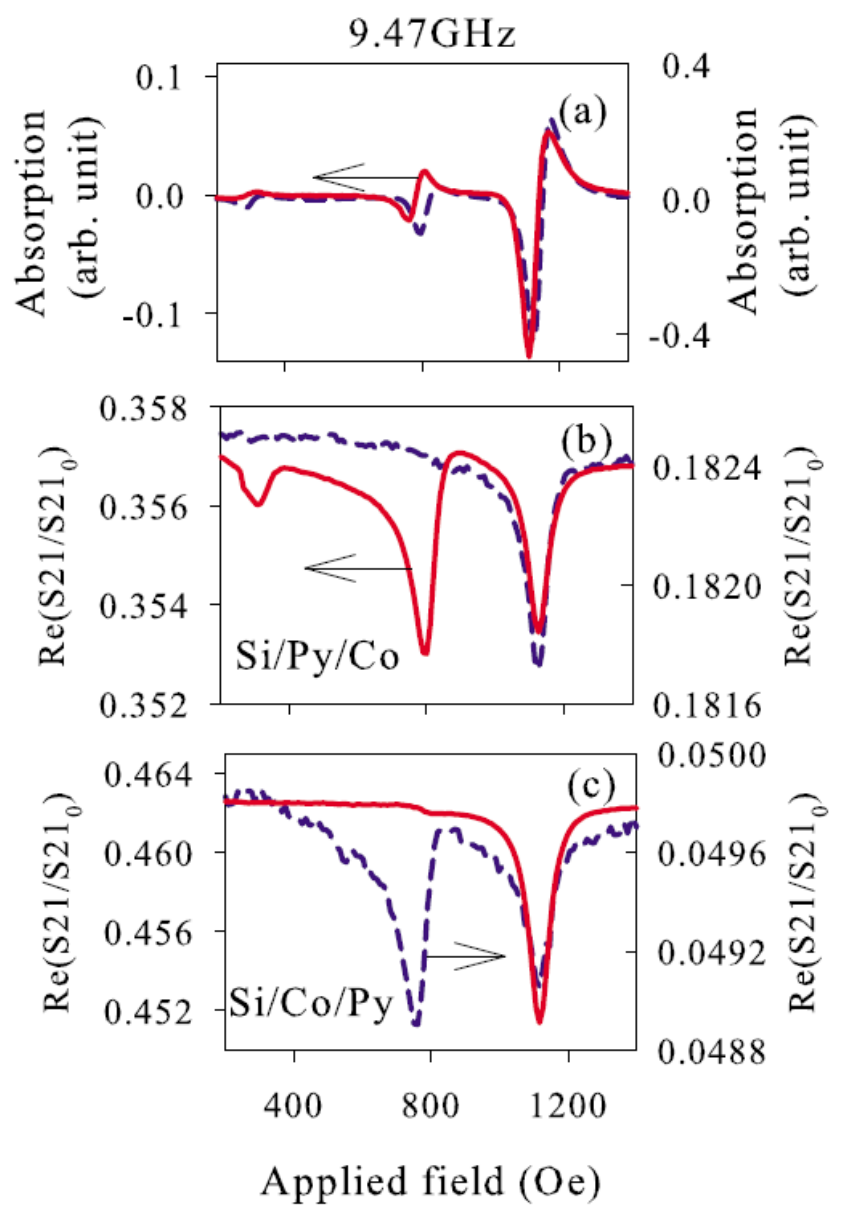

FIG. 27. (a) Cavity FMR data for the magnetic Si/Co/Py and $\mathrm{Si} / \mathrm{Py} / \mathrm{Co}$ bi-layers. Red solid line $\mathrm{Si} / \mathrm{Co} / \mathrm{Py}$, blue dashed line: $\mathrm{Si} / \mathrm{Py} / \mathrm{Co}$. (b, c) BFMR traces for $\mathrm{Si} / \mathrm{Py} / \mathrm{Co}$ and $\mathrm{Si} / \mathrm{Co} / \mathrm{Py}$, respectively. Red solid lines in (b, c) - film faces the transducer; blue dashed lines in (b, c) - substrate faces the transducer. Frequency is $9.47 \mathrm{GHz}$. From [88].

In Ref. [88], similar bi-layer Co/Permalloy structures were characterised, but now with the stripline BFMR. A wide $(w=1.5 \mathrm{~mm})$ microstrip transducer was used. As shown in Fig. 7, the microwave field of the microstrip line is largely uniform above the microstrip over the distance $w$ from its surface. Thus, given the substrate thickness of $0.9 \mathrm{~mm}$, using a microstrip line with $w=1.5 \mathrm{~mm}$ allows one to study the same sample orientation dependence on the FMR response.

Measurements were taken on bilayer samples with similar composition: Si $\backslash \mathrm{Ta}[5 \mathrm{~nm}] \backslash \mathrm{Py}[80 \mathrm{~nm}] \backslash \mathrm{Co}[10 \mathrm{~nm}] \backslash \mathrm{Ta}[5 \mathrm{~nm}] \quad$ [denoted as Si $\backslash \mathrm{Py} \backslash \mathrm{Co}$ in Fig. 27(b)] and $\mathrm{Si} \backslash \mathrm{Ta}[5 \mathrm{~nm}] \backslash \mathrm{Co}[10 \mathrm{~nm}] \backslash \mathrm{Py}[80 \mathrm{~nm}] \backslash \mathrm{Ta}[5 \mathrm{~nm}]$ [denoted as Si $\backslash \mathrm{Co} \backslash \mathrm{Py}$ in Fig. 27(c)]. From these panels one sees that the stripline FMR responses of the SilCo\Py and Si Py $\backslash$ Co structures are fundamentally different. This is in contrast to results of the reference microwave cavity measurements [Fig. 27(a)], which suggest that the two samples are magnetically very similar to each other. Hence, what is important for the FMR response is the orientation of the layers with respect to the stripline. Similarly to the waveguide data in Fig. 26, each time the Co layer faces the stripline, the $1^{\text {st }}$ SSWM dominates in the spectrum. On the contrary, this peak is of negligible amplitude when the Permalloy layer faces the transducer. One also notices that the amplitude of the FMR response of the $1^{\text {st }}$ SSWM taken with the cavity FMR is smaller than in the "Co facing" cases but larger than in the "Permalloy facing" cases. 
Figure 28 demonstrates the absorption traces measured for two sets of similar Co-Py bilayer films. The differences between the samples from one set was the thickness of the Py layer and the difference between the sets was the layer ordering. As seen from Fig. 28, similar to Fig. 27, each time the Co layer faces the stripline, the amplitude of the $1^{\text {st }}$ SSWM is very strong. However, when the Co layer faces the microwave field the amplitude of the $1^{\text {st }}$ SSWM is negligible. One also notices that the amplitude of the $1^{\text {st }}$ SSWM for the "Co facing configuration" strongly depends on the frequency such that this mode becomes dominant in the spectrum at the largest frequency in this experiment $-18 \mathrm{GHz}$.

$7.5 \mathrm{GHz}$

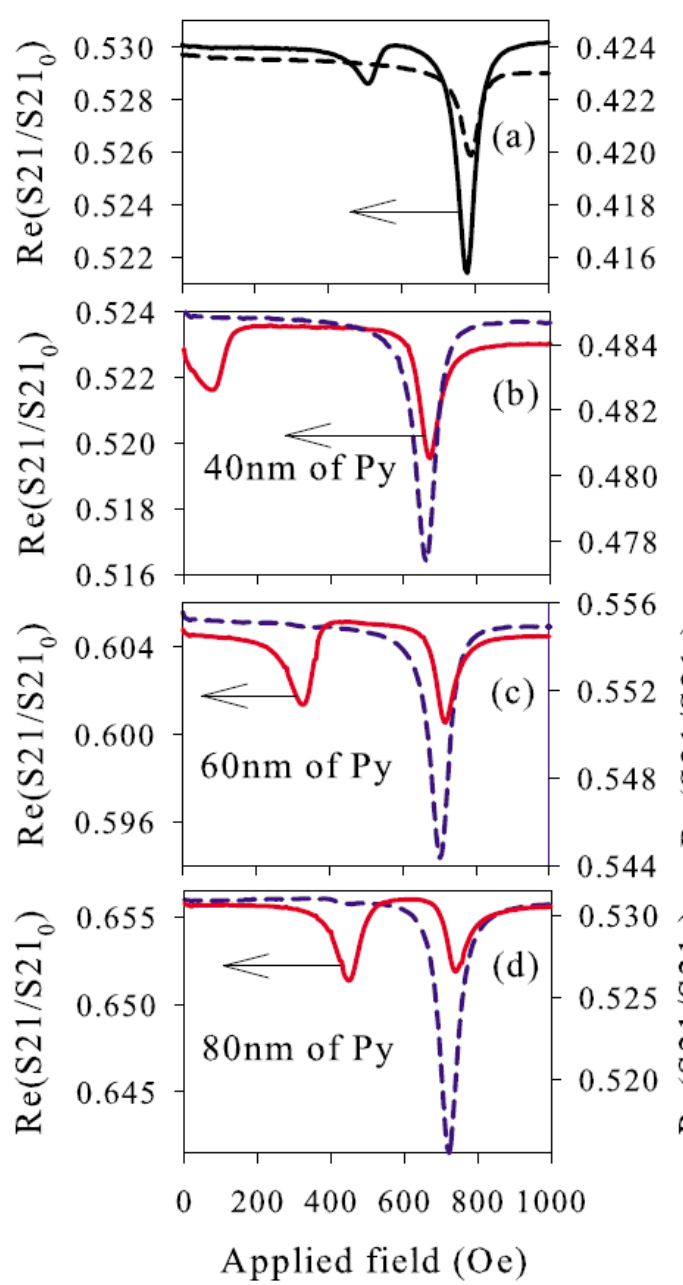

$18 \mathrm{GHz}$

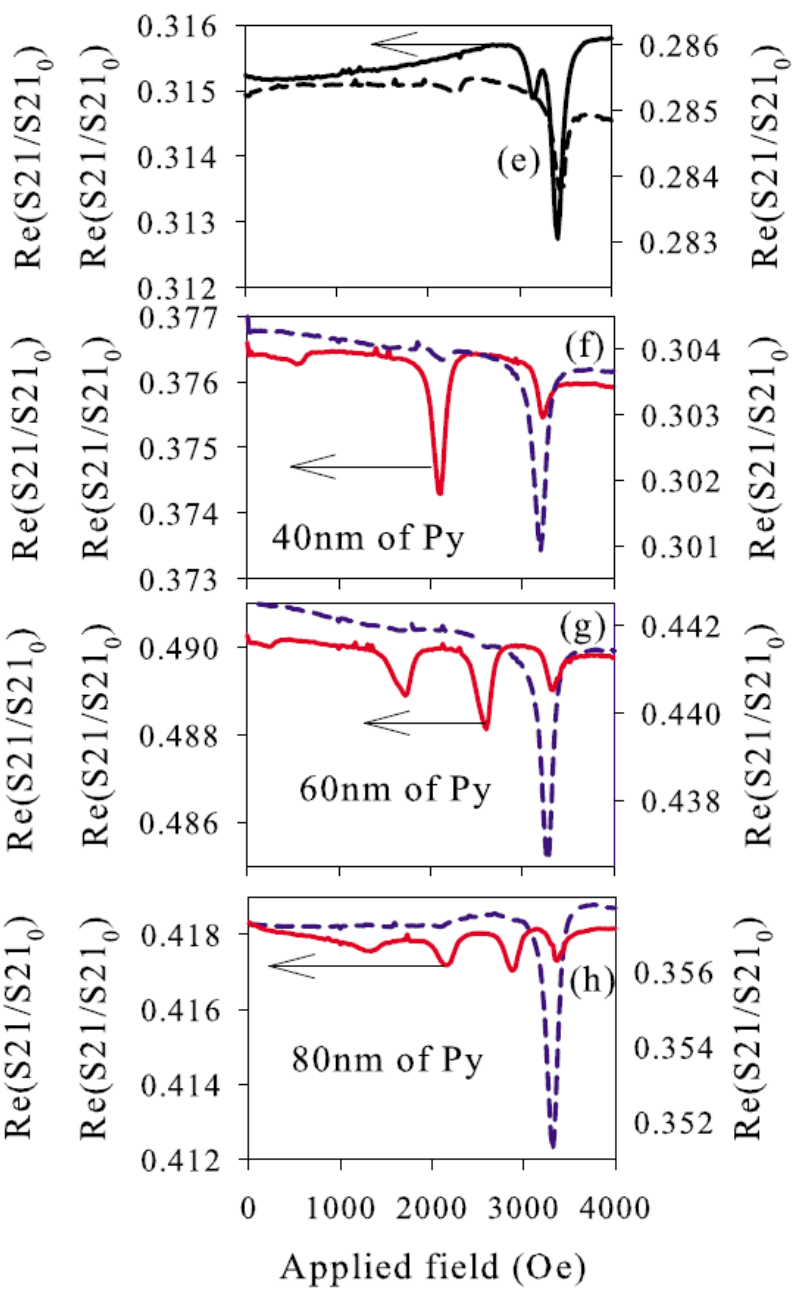

FIG. 28 Broadband FMR absorption spectra for single-layer Permalloy films (a, e) and Co-Py bilayers on Si substrates $(\mathrm{b}-\mathrm{d}, \mathrm{f}-\mathrm{h})$. The driving microwave frequency is (a-d) $7.5 \mathrm{GHz}$ and $(\mathrm{e}-\mathrm{h}$ ) $18 \mathrm{GHz}$. Panels (a, e): results for $80 \mathrm{~nm}$-thick (solid line) and $40 \mathrm{~nm}$-thick (dashed line) singlelayer Permalloy films are given for comparison. Panels $(b-d, f-h)$ : red solid line - Co layer faces the stripline, dashed blue line - Py layer faces the stripline. In all cases the thickness of the Co layer is $10 \mathrm{~nm}$; the thickness of the Py layer is indicated in the panels. From [88].

\subsubsection{Film-flip FMR of a weakly-conducting bi-layer film}

The third and the last experiment that has to be highlighted is the stripline BFMR measurement of a weakly conducting bi-layer material $\mathrm{La}_{1-\mathrm{x}} \mathrm{Sr}_{\mathrm{x}} \mathrm{MnO}_{3} / \mathrm{BiFeO}_{3}$ (LSMO/BFO) film grown with pulsed laser deposition technique on a strontium oxide substrate [186]. LSMO is a 
weakly conducting half-metal and BFO is a multiferroic antiferromagnet. No difference in FMR responses was observed for the two film orientations: the film facing the stripline and substrate facing the stripline. Furthermore, both in-plane and perpendicular-to-plane FMR traces demonstrated negligible amplitudes of the $1^{\text {st }}$ higher-order SSWM (Fig. 29).

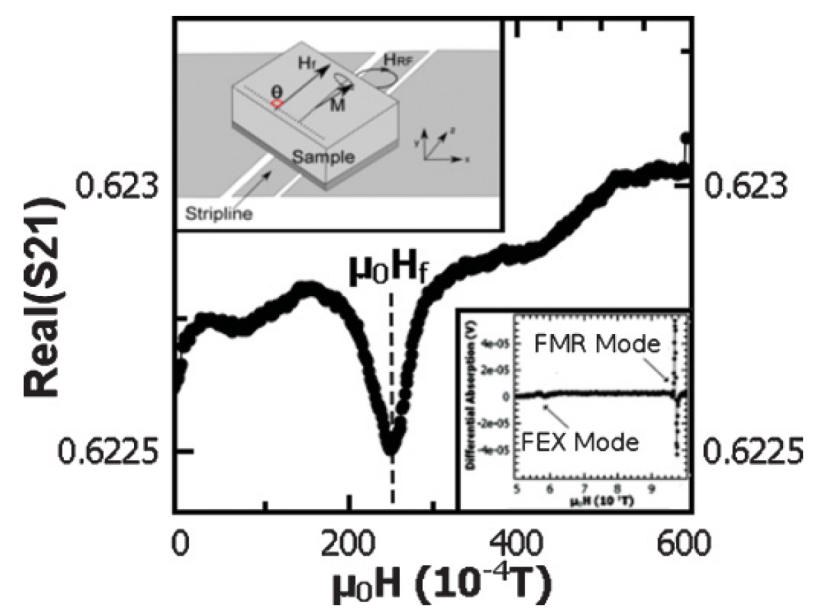

FIG. 29 Raw in-plane VNA-BFMR data for a LSMO $\left(38.86 \mathrm{~nm}\right.$ ) film in the $0^{\circ}$ orientation (see the upper inset) with a $3 \mathrm{GHz}$ driving microwave film. The bottom inset displays perpendicular-toplane field-modulated BFMR data on a $\operatorname{LSMO}(54.8 \mathrm{~nm}) / \mathrm{BFO}(23 \mathrm{~nm})$ sample at a $14 \mathrm{GHz}$ driving frequency. From [186].

\subsubsection{Qualitative explanation of transmission/reflection and film-FMR experiments}

The fact that the film with a small electric conductivity shows completely different behaviour compared to highly-conductive films suggests that the electric conductivity of metals is important for explaining the data in Figs. 26 and 27. While placed on top of a microwave line, metallic magnetic films behave similar to the same films but exposed to a normally incident electromagnetic wave [Fig. 26(b, c, e)]. In the waveguide experiment, the direction of the microwave flux is well defined: it is from the microwave generator towards the film surface. Hence, the difference between the traces in Fig. 26(e) is due to single-surface incidence of the microwave flux on the film surface. The comparison of Fig. 26 and Fig. 27 suggests that in Fig. 27 we are also dealing with the exposure of just one surface of the films to the microwave magnetic field. This field is the microwave Oersted field of the microwave current in the stripline.

As already discussed above, the microwave magnetic field of a stripline induces a microwave eddy current inside a metallic film sitting on top of the stripline. For the films with subskin-depth thicknesses the density of the eddy current is perfectly uniform across the film thickness. This also explains the qualitatively different behaviour seen for the same samples in the cavity FMR [Fig. 27(a)]. For simplicity, let us consider a stripline of an infinite width. Then the eddy-current density distribution in the film plane is also uniform. Importantly, the phase of the current changes by 180 degree when the sample surface, onto which the microwave magnetic field is incident, is changed to the opposite one [Fig. 30(a,b)]. This result follows from symmetry reasons and also can be easily obtained from the circulation law for the magnetic field (i.e. from the respective Maxwell's equation in integral presentation). 


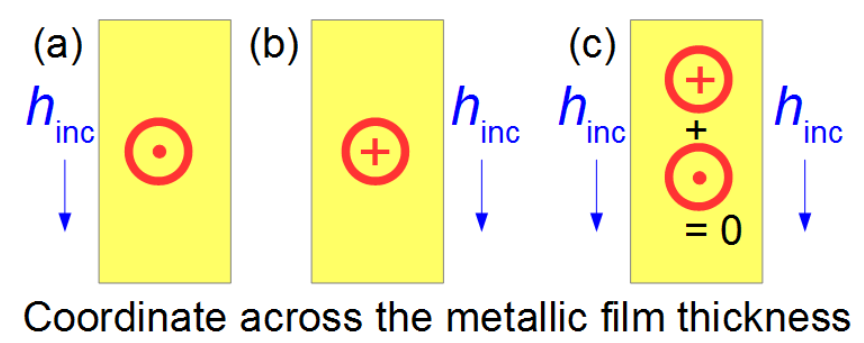

FIG. 30 Schematic picture explaining the excitation of eddy currents $j$ in conducting samples. The directions of the currents are indicated by the red dot representing the arrowhead and the red cross, which represents the tail of the arrow. (a) and (b) the microwave field $h_{\text {inc }}$ is incident only on one of the film surfaces. (c) The microwave field $h_{\text {inc }}$ is incident on both film surfaces. Note that this picture is valid for metal layers with sub-skin-depth thicknesses only.

In the cavity FMR experiment, a sample is placed in a microwave cavity, right in its centre where the microwave magnetic field is perfectly uniform. This implies that the microwave magnetic field is incident on both sample surfaces with the same amplitude and phase [Fig. 30(c)]. Consequently, the two partial eddy currents excited by the fields, incident onto the right-hand and left-hand film surfaces, cancel each other. Thus, in the cavity FMR experiment the metallic films of sub-skin-depth thicknesses behave as magneto-insulators and the experimental FMR response in Fig. 27(a) is essentially a response of a magnetically insulating bi-layer film.

\section{Theory of the broadband stripline FMR of highly conducting films}

In this section, we overview the theory of eddy current contribution to the stripline FMR response of metallic ferromagnetic materials. As has been mentioned in Section 5.3.4, an important peculiarity of the stripline FMR is the single-surface incidence of the microwave magnetic field on the sample.

Our analysis will be based on the semi-analytical approach from [90] since it is the most suitable for understanding of the experiment on the bi-layer films lacking inversion symmetry. It also makes it possible to extend the theory onto other practically important cases of films which are non-uniform across their thickness, for instance films with surface and interface anisotropies. Such an extension is a significant problem for other methods, especially for analytical ones. However, before we start presenting this theory, it is useful to mention previous analytical theories. One of them is the work [187] in which the authors derived an analytical expression for a surface impedance of a single-layer metallic ferromagnetic film placed in a microwave field whose amplitudes are different at the two film surfaces. Basically, as follows from the previous section, by setting the amplitude of the field at one of the surfaces to zero one should retrieve the case of a strip line of an infinite width. This has been realised very recently by the authors of Ref. [188] who used this theory to explain their experimental results.

One more theoretical paper which is worth mentioning in this regard is Ref. [189]. In this work, an approximate theory of transmission of a plane electromagnetic wave through a tri-layer metallic ferromagnetic film was developed. The final formulae implicitly demonstrate a strong impact of the microwave eddy-currents on the microwave magnetisation dynamics in these materials. Again, because the aim of this work was quite different, the authors do not discuss possible implications of their result for the stripline FMR.

The paper which consistently addressed the theory of the eddy-current contribution to the stripline FMR response of metallic ferromagnetic materials is Ref. [90]. The main finding of this 
paper is the importance of the boundary condition for the microwave magnetic field at the surface of the film facing away from the stripline [see Eq. (49)]. Indeed, in order to eliminate the contribution of travelling spin waves to the FMR line width (see Fig. 17 and its discussion), the stripline width should be macroscopic. As we have shown in Fig. 24, a strong shielding effect by microwave eddy currents takes place in this case, and Eq. (49) is a good approximation for the microwave magnetic field at $y=L$ (i.e. at the film surface facing away from the stripline, see the frame of reference in Fig. 6(a)). On the other hand, Eq. (49) represents an exact result for $k=0$ [see Eq. (57)]. Recall, the case of $k=0$ is equivalent to a stripline of an infinite width $(w \rightarrow \infty)$. Thus, as long as the stripline is wide enough such that the strong shielding by the eddy currents in metallic ferromagnetic films takes place, one can use the approximation of an infinitely wide stripline. This assumption reduces the problem to one dimension and justifies the use of the simple boundary condition Eq. (49).

Note that Eq. (51) and, consequently, Eq. (49) implicitly imply a film substrate made from a highly insulating material with the usual value of dielectric permittivity, e.g. intrinsic silicon ( $\varepsilon=11$ ), gadolinium-gallium garnet $(\varepsilon=14)$, or glass $(\varepsilon=6)$. If the substrate is conducting, e.g., made of highly doped $\mathrm{Si}$, due to its significant thickness (typically $0.5-1 \mathrm{~mm}$ ) it might be important to include its complex permittivity into the boundary condition. (This was the case in the experiment [188]). In this case the boundary condition from [174]

$$
h_{\mathrm{x}}^{(2)}\left(y=L^{-}\right)=-\frac{c_{0} \varepsilon_{0} \varepsilon}{\sigma} \frac{\partial h_{\mathrm{x}}^{(2)}\left(y=L^{-}\right)}{\partial y}
$$

is more appropriate, where $\varepsilon$ is the complex electric conductivity of the substrate and $\sigma$ is the conductivity of the ferromagnetic layer. For $\varepsilon=0$ Eq. (59) reduces to Eq. (49).

The presence of the microwave magnetic field of the stripline transducer is taken into account in a way, similar to the derivation of Eq. (49): the transducer is modelled as an infinitely thin sheet carrying a microwave current with the current density $j$, and located at $y=0$, i.e. infinitesimally close to the surface of the film, but not in a direct electric contact to the film. (Obviously, in real experimental situations this approximation is valid for $d<<w$, where $d$ is the sample separation from the stripline surface.) This boundary condition reads

$$
h_{\mathrm{x}}\left(y=0^{+}\right)=-j_{\mathrm{z}} \text {. }
$$

Importantly, the boundary conditions Eqs. $(59,60)$ are for the fields inside the film. This excludes the areas in front of the film and behind it from consideration and makes the numerical code very simple and extremely fast.

The physical description of the problem is given by Maxwell's equations in 1D for the microwave fields and the LLG equation for the magnetisation vector. Within the film we have

$$
\begin{gathered}
\nabla \times \mathbf{h}=\sigma \mathbf{e}, \\
\nabla \cdot \mathbf{h}=-\nabla \cdot \mathbf{m}, \\
\nabla \times \mathbf{e}=-i \omega \mu_{0}(\mathbf{h}+\mathbf{m}),
\end{gathered}
$$

where $\mathbf{m}$ is the dynamic magnetisation, $\mathbf{h}$ and $\mathbf{e}$ are the microwave magnetic and electric fields, respectively, $\sigma$ is the electric conductivity, and $\omega$ is the angular frequency. To obtain Eqs. (61), we have assumed that all dynamic fields and the dynamic magnetisation have the time dependence in the form $\exp (i \omega t)$.

In the one-dimensional case and the frame of reference from Fig. 6(a) one finds 
$\partial / \partial x=\partial / \partial z=0$, and Eq. $(61)$ reduces to

$$
\frac{\partial^{2} h_{x}}{\partial y^{2}}+i \delta^{2} h_{x}=-i \delta^{2} m_{x}
$$

where $\delta=\left(\mu_{0} \sigma \omega\right)^{1 / 2}$ and $\sqrt{ } 2 / \delta$ is the classical skin depth. One sees that for a non-magnetic film this equation reduces to Eq. (55) for $k=0$. This evidences that all the eddy-current analysis of the nonmagnetic films should be largely applicable to the ferromagnetic ones (see above).

Equation (62) is solved numerically together with the linearised Landau-Lifshitz-Gilbert (LLLG) equation

$$
i \omega \mathbf{m}+\gamma\left\{H_{\mathrm{C}}\left(\mathbf{i}_{\mathrm{x}} m_{x}+\mathbf{i}_{\mathrm{y}} m_{\mathrm{y}}\right) \times \mathbf{i}_{\mathrm{z}}-M_{\mathrm{s}}\left[\mathbf{i}_{\mathrm{x}} \frac{2 A}{M_{\mathrm{s}}^{2}} \frac{\partial^{2} m_{x}}{\partial y^{2}}+\mathbf{i}_{\mathrm{y}}\left(h_{y}+\frac{2 A}{M_{\mathrm{s}}^{2}} \frac{\partial^{2} m_{\mathrm{y}}}{\partial y^{2}}\right)\right] \times \mathbf{i}_{\mathrm{z}}\right\}=-\gamma M_{\mathrm{s}} h_{\mathrm{x}}\left(\mathbf{i}_{\mathrm{z}} \times \mathbf{i}_{\mathrm{x}}\right)
$$

and the equation $h_{\mathrm{y}}=-m_{\mathrm{y}}$, which follows from the second equation of the system Eq. (61). In Eq. (63), $M_{\mathrm{s}}$ is the saturation magnetisation, $A$ is the exchange constant, $H_{\mathrm{C}}=H+i \alpha_{\mathrm{G}} \omega / \gamma$, being $\alpha_{\mathrm{G}}$ the Gilbert magnetic damping, and $\mathbf{i}$ are the unit vectors along the coordinate axes. (The static magnetic field $\mathbf{H}$ and the equilibrium magnetisation point along the $z$-axis). Different ferromagnetic layers of the multi-layered structures may have different $M_{\mathrm{s}}, A$ and $\alpha_{\mathrm{G}}$.

The boundary conditions Eq. (60) and Eq. (49) are applied at the front and far surfaces of the film ( $y=0$ and $y=L$ respectively). For instance, for the case of a bi-layer film from [90] $L=L_{1}+L_{2}$, where $L_{1}$ and $L_{2}$ are the thicknesses of the ferromagnetic layers. One also needs electromagnetic boundary conditions at the interface of two metallic ferromagnetic magnetic layers. The interface is located at $y=L_{1}$. From the condition of continuity of $h_{\mathrm{x}}$ and $e_{\mathrm{z}}$ at the interface one finds

$$
\sigma_{2} \frac{\partial h_{\mathrm{x}}^{(1)}}{\partial y}=\sigma_{1} \frac{\partial h_{\mathrm{x}}^{(2)}}{\partial y}, h_{\mathrm{x}}^{(1)}=h_{\mathrm{x}}^{(2)}
$$

where the indices ' 1 ' and ' 2 ' denote the layer numbers. On top of this, the presence of the differential operator of the effective exchange field in Eq. (63) requires application of boundary conditions for the dynamic magnetisation at the two surfaces of the film and also at the interfaces between the ferromagnetic layers. The interface boundary conditions are given by Eqs. (6-10).

The approach from [90] to the numerical solution of Eqs. $(62,63)$ is based on a finitedifference discrete model. Whereas Eq. (62) is discretised using a standard three-point formula [190], the implementation of the aforementioned boundary conditions and the exchange operator $2 A / M_{\mathrm{s}}^{2} \partial^{2} \mathbf{m} / \partial y^{2}$ requires special attention since such peculiarities are often overlooked in textbooks on finite-difference methods. For more details we refer the interested reader to the original paper [90].

The numerical solution delivers the amplitudes of the two components of the dynamic magnetisation $-m_{\mathrm{x}}(\mathrm{y})$ and $m_{\mathrm{y}}(y)-$ and of the microwave magnetic field $h_{\mathrm{x}}(y)$ on an equidistant mesh along $y$. The stripline response is derived from $h_{\mathrm{x}}(y=0)$. To some extent, the procedure is similar to the practical situation of the parameter extraction from experimental FMR data (Section 3). The complex linear impedance $Z_{\mathrm{r}}$ of a stripline loaded with a ferromagnetic film is the quantity that characterises the efficiency of microwave absorption in the BFMR. We recall that the width of the stripline is assumed to be infinite in the calculation above. However, the technique outlined below allows one to treat the finite-width striplines, too, based on the numerical value of $h_{\mathrm{x}}(y=0)$ 
obtained in the approximation of $w=\infty$. According to Ref. [166], for the in-plane homogeneous microwave electric field

$$
Z_{\mathrm{r}}=\frac{e_{z}(y=0)}{j_{\mathrm{z}}^{*} w} .
$$

where $w$ is the width of the stripline. The microwave electric field is easily obtained from the numerical solution of the equation $e_{z}=-\frac{1}{\sigma} \partial h_{x} / \partial y$. The value of $Z_{\mathrm{r}}$ has to be transformed into values of the scattering coefficient $S_{21}$. The film sample has the length $l$ along the $\mathrm{z}-$ axis and sits on top of the stripline. Usually the sections of the stripline ("unloaded stripline"), which are not covered by the sample, have the characteristic impedance $z_{0}=50 \Omega$. $Z_{\mathrm{f}}$ is the characteristic impedance of the section of the stripline loaded by the sample ("loaded stripline")

$$
Z_{\mathrm{f}}=\sqrt{\frac{Z_{0}+Z_{\mathrm{r}}}{Y_{0}}},
$$

where $Z_{0}$ and $Y_{0}$ are the complex series resistance and the complex parallel conductance of the unloaded stripline. $\gamma_{\mathrm{f}}$ is the complex propagation constant of the loaded stripline

$$
\gamma_{\mathrm{f}}=\sqrt{\left(Z_{0}+Z_{\mathrm{r}}\right) Y_{0}} \text {. }
$$

The transmission matrix $T$ of the loaded stripline is

$$
T=\left[T^{(1)} T^{(2)} T^{(3)}\right],
$$

where $T^{(1)}$ and $T^{(3)}$ are the transmission matrices of the junctions of the loaded stripline with the unloaded stripline. These matrices account for losses that are inserted due to partial reflection of the incident electromagnetic wave back. These reflections are defined via the complex reflection coefficient

$$
\Gamma= \pm \frac{Z_{\mathrm{f}}-z_{0}}{Z_{\mathrm{f}}+z_{0}},
$$

where the positive sign is for the front edge and the negative sign is for the rear edge of the sample. After some algebra the scattering parameter $S_{21}$ of the whole loaded stripline is

$$
S_{21}=\frac{\Gamma^{2}-1}{\Gamma^{2} e^{-\gamma_{\mathrm{f}} l}-e^{\gamma_{\mathrm{f}} l}} .
$$




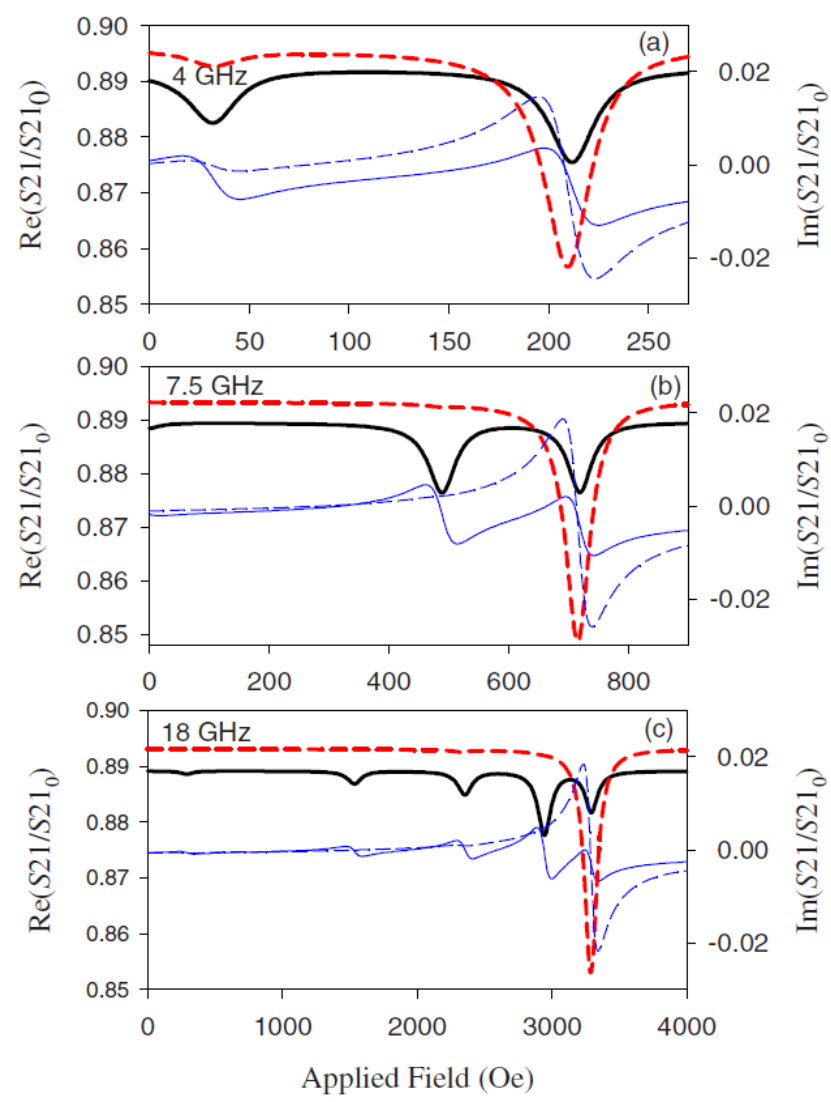

FIG. 31 The ratio $S 21 / S 21_{0}$ for a bi-layer Co-Permalloy (Co-Py) ferromagnetic metallic film one centimetre long. The microwave frequency is (a) $4 \mathrm{GHz}$, (b) $7.5 \mathrm{GHz}$, and (c) $18 \mathrm{GHz}$. Thick lines: real parts of $S 21 / S 21_{0}$ (left axis), thin lines: imaginary parts of $S 21 / S 21_{0}$ (right axis). Solid lines: the Co layer faces the stripline, dashed lines: the Py layer faces the stripline. From [90].

The results of numerical calculations from [90] using this formalism for the frequencies $\omega /(2 \pi)=4$, 7.5, and $18 \mathrm{GHz}$ are shown in Fig. 31. A Co-Permalloy (Co-Py) bilayer film with the same parameters as in the experimental paper [88] was considered. The width of the microstrip line $w=1.5 \mathrm{~mm}$, and the relative electric permittivity of the substrate used to calculate the characteristics of the unloaded microstrip line [Eq. (66)] $\varepsilon_{\text {sub }}=3.5$. From Fig. 27 one sees that the FMR absorption when the Co layer faces the stripline is a few times smaller than when the Permalloy later faces it. One also sees that the amplitude of the $1^{\text {st }}$ higher-order SSWM (the second peak from the right) is visible only for Co facing the stripline. The amplitude of this peak grows with frequency and becomes larger than that of the fundamental mode (the most right-hand peak) at higher frequencies. This behaviour agrees well with the results of the microstrip BFMR experiment in Fig. 28. Furthermore, this theory explains well the experimental data obtained with a coplanar transducer too (see Fig. 4 in [88]).

This theory confirms that the strong dependence of the stripline FMR response of a metallic ferromagnetic film lacking inversion symmetry on the sample orientation is due to the microwave magnetic shielding by eddy currents. Indeed, the calculated profile $h_{\mathrm{x}}(y)$ in Fig. 32 is similar to ones in Figs. 21 and 24. Within each of the metallic layers it is linear and the field vanishes at $y=L$. The only qualitative difference between Fig. 21 and Fig. 32(c) is a non-monotonic change in the slope at the interface of the two layers. The abrupt change in the slope is due to the difference in the layer conductivities: $\sigma_{\mathrm{Co}}=4 \sigma_{\mathrm{Py}}$. Accordingly, the eddy current density in Co is larger by $\sim 4$ times than that in Permalloy. 
The upper panel [Fig. 32(a)] shows the distribution of the dynamic magnetisation $m_{\mathrm{x}}(y)$ across the bilayer thickness. One observes a considerably inhomogeneous $m_{\mathrm{x}}(y)$-distribution through the Py layer for the fundamental mode with the minimum at the layer interface. This represents a clear signature of partial pinning of magnetisation at the Py interface with Co. One also sees that the first higher-order mode of the whole bilayer is a combination of the fundamental mode of the Co layer and the first higher-order SSWM of the Py layer.

The $m_{\mathrm{x}}(y)$-distribution in this panel is shown for the Co-facing film orientation. For the surface current applied at $y=L_{1}+L_{2}$ ("Py-facing orientation") one obtains the mirror image of Fig. 32(a). The same profiles for the Co-facing configuration, but for $18 \mathrm{GHz}$ are shown in the middle panel. One sees that they are very similar to those in Fig. 32(a). The main observation from these figures is that both $\mathbf{m}(y)$ and $\mathbf{h}(y)$ are strongly asymmetric functions of $y$. Furthermore, from the r.h.s part of Eq. (63) one sees that the magnetisation precession in the film is driven by the total microwave magnetic field in the material. The latter is a combination of the microwave Oersted field of the stripline, which is uniform on the length scale of the film thickness, and the Oersted field of the eddy current induced in the film by the stripline field (see the sketches in Fig. 21).

Since the driving field is strongly non-uniform it is not surprising that the FMR response amplitude for plain materials lacking inversion symmetry strongly depends on the sample orientation with respect to the stripline. A straightforward consequence of this conclusion is an idea to try to solve Eq. (63) separately from Eq. (62) assuming that the profile of the microwave driving field is given. A suitable candidate for the field profile is the one for a non-magnetic bi-layer film with the same conductivities as for the ferromagnetic layers. This profile can be easily obtained from the analytical solution of Eq. (62) with the vanishing r.h.s. and the boundary conditions on the film surfaces Eqs. $(49,60,64)$. The profile calculated in this way is actually quite similar to one in Fig. 32(c). The only major difference between the two is the slight deviation from the straight lines in the field profile seen in Fig. 32(c), whereas the respective non-magnetic film would show a profile consisting from two sections of perfectly straight lines. The solution of Eq. (63) assuming a given r.h.s in this form results in a qualitatively similar behaviour to one shown in Fig. 31. However, quantitatively the results of the rigorous calculation in Fig. 31 and of this approximate approach are noticeably different. Hence, one needs to solve the system of Eqs. $(62,63)$ selfconsistently to get good quantitative agreement with experiment. 


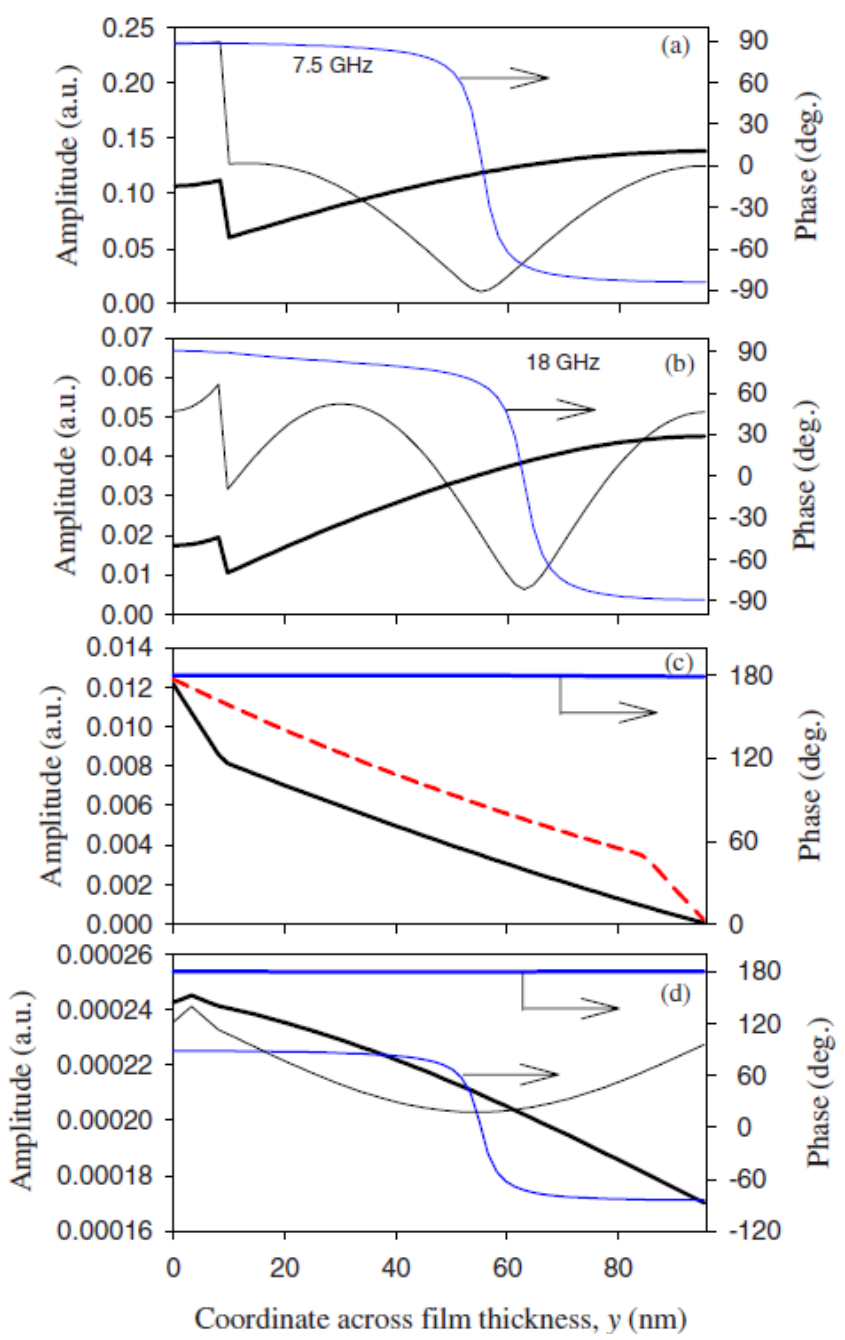

FIG. 32 Distributions of the dynamic magnetisation and dynamic magnetic and electric fields. (a) In-plane component of the dynamic magnetisation $m_{\mathrm{x}}$ at $7.5 \mathrm{GHz}$. (b) The same, but for $18 \mathrm{GHz}$. (c) In-plane dynamic magnetic field $h_{\mathrm{x}}$ for the fundamental mode. (d) Dynamic electric field $e_{\mathrm{z}}$ for the fundamental mode. In all panels, the thick lines are for the fundamental mode and the thin lines are for the first higher-order SSWM. The black solid lines are for the Co layer facing the stripline. The dashed red line is for the Py layer facing the stripline. Blue lines - phase (right axis). From [88].

One more important question is the strength of coupling of the resonance in the sample to the stripline. From the theory of the cavity FMR it is known that in order to measure an undistorted FMR response it is necessary to ensure that coupling of the sample to the cavity is weak. If the coupling is strong, one measures characteristics of a coupled system - sample/cavity instead. The characteristics of the coupled oscillations are essentially different from the intrinsic FMR response of the sample. For instance, the resonance frequency may be strongly shifted (see, e.g., [85]). For a weaker but still considerable coupling one may expect the presence of extra FMR losses - "loadedresonance" losses. For this reason, in the cavity FMR it is important to keep the sample volume as small as possible and the cavity volume as large as possible. This weakens the coupling by decreasing the filling factor of the cavity by the sample.

From this point of view, one may expect a very strong coupling of the sample to the probing device - the stripline - in the stripline FMR. This is because the sample sits directly on top of the stripline and may occupy a significant part of its length. However, it may be shown that the excitation of the eddy currents efficiently decouples the FMR dynamics from the microwave 
currents in the stripline. (For a relevant discussion also see Ref. [191]). Therefore, for highly conducting materials of sub-skin-depth thicknesses, such as metal films, the coupling of the ferromagnetic resonance is always weak and no loaded-resonance losses are expected to contribute to the measured resonance line width [192]. However, for magneto-insulating films this mechanism of decoupling is not present and one has to be careful while measuring responses of thick and lowmagnetic-loss materials in this case.

As for the resonance frequency/field shift due to a strong coupling [85], this problem is naturally absent in the stripline FMR due to the broadband nature of the probing device.

\section{Possible applications of the strong microwave shielding effect contribution to the stripline FMR response.}

As follows from the experiment and the theory above, the main effect of the excitation of the eddy currents is the strong orientational dependence of the stripline FMR response for films lacking inversion symmetry. On the other hand, the theory shows that the contribution of the eddy currents to the mode eigen-frequencies/resonant fields and resonant line widths remains negligible as long as the films are significantly thinner than the microwave skin depth.

Also the theory demonstrates that the higher-order standing spin waves in single-layer metallic films should be excited more efficiently in the stripline FMR than in the cavity FMR. Indeed, if a film is magnetically uniform in the direction of its thickness, the higher-order SSWMs are not excited in the highly uniform microwave magnetic field of a cavity [69]. As explained in Section 5, in the cavity FMR experiments the metallic films with sub-skin-depth thicknesses behave as the insulating ones. This is because the microwave magnetic field is incident with the same amplitude on both surfaces of the films. This creates a highly symmetric situation, which leads to the vanishing amplitudes of the higher-order modes.

The single-side incidence of the microwave magnetic field on the film surface breaks this symmetry. Hence, it becomes theoretically possible to excite the $1^{\text {st }}$ SSWM and also the higherorder modes for a perfectly magnetically uniform single-layer film in the stripline FMR. The possibility of excitation of higher-order modes is very important, since their frequency/resonantfield positions carry information about the exchange constant for the material. The FMR is the best way to extract this information, provided the higher-order modes are excited (see Ref. [186] and references therein). Thus, with the stripline FMR one can potentially measure the value of the exchange constant for highly conducting ferromagnetic materials which do not display higher-order peaks in the FMR traces taken with the traditional method - the cavity FMR.

Figure 33 shows results of the simulation of PP FMR absorption traces for a $40 \mathrm{~nm}$-thick single-layer film with unpinned surface spins for three different values of material electrical conductivity: $\sigma_{\mathrm{Py}} / 100, \sigma_{\mathrm{Py}} / 10$, and $\sigma_{\mathrm{Py}}$, where $\sigma_{\mathrm{Py}}=4.5 \times 10^{6} \mathrm{~S} / \mathrm{m}$ is the standard electrical conductivity of Permalloy. From Fig. 33 one clearly sees that the amplitude of the $1^{\text {st }}$ SSWM drops with a decrease in the material electric conductivity. Thus, it should be possible to excite the higher-order SSWMs in these perfectly thickness-uniform materials, provided the sample conductivity is large enough. 

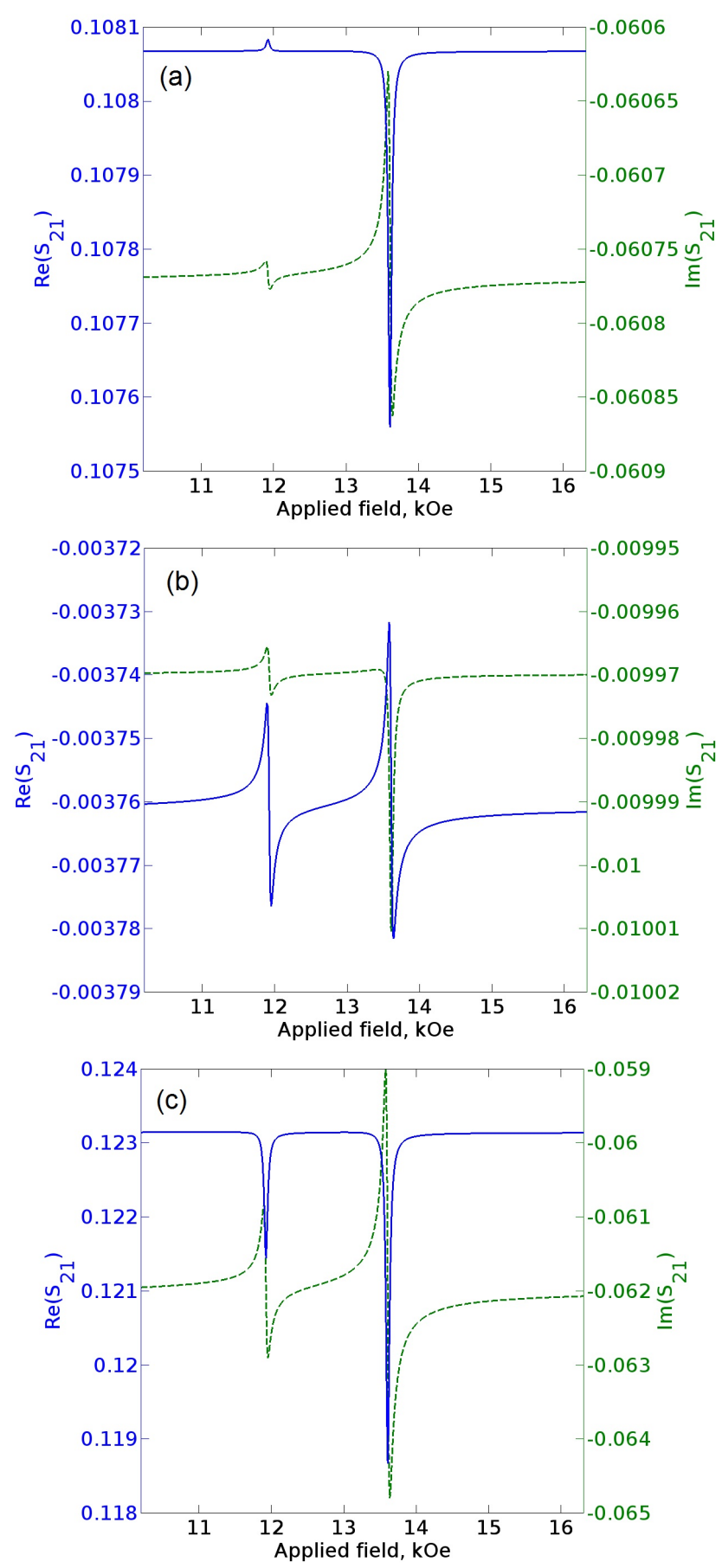

FIG. 33. Simulated stripline PP FMR responses for a $40 \mathrm{~nm}$-thick film with magnetic parameters of Permalloy for three different values of material electrical conductivity: (a) $\sigma_{\mathrm{Py}} / 100$, (b) $\sigma_{\mathrm{Py}} / 10$, and (c) $\sigma_{\mathrm{Py}}$, where $\sigma_{\mathrm{Py}}=4.5 \times 10^{6} \mathrm{~S} / \mathrm{m}$ is the standard electrical conductivity of Permalloy. Frequency is $10 \mathrm{GHz}$.

However, very often single-ferromagnetic-layer materials are not thickness uniform. One of the possible reasons for this is the spontaneous development of surface magnetic anisotropy by these films. Most often this anisotropy is normal uni-axial (or perpendicular) - often called "Perpendicular magnetic anisotropy (PMA)". There are different reasons for the PMA formation. One of them is a mechanical stress at an interface of a ferromagnetic layer with a non-magnetic seed or capping layer. Interfaces with Pt, Pd or Ta are very known examples (see, e.g., [29, 41, 42, 47, 139] and references therein). Another reason for the PMA formation is oxidation of the (uncapped) film surface in the ambient atmosphere [193]. 
In Ref. [121] we showed that the stripline FMR is an excellent tool to detect hidden magnetic non-uniformities in the ferromagnetic materials. Two possible FMR protocols - "Filmflip Stripline" and "Stripline vs. Cavity" - were simultaneously demonstrated. In order to use these protocols to detect the presence of a magnetic non-uniformity one has to compare results of stripline FMR measurements of the material for the two film orientations - with the film facing the stripline ("unflipped" orientation) and the substrate of the film facing the stripline ('flipped' orientation). This protocol is "Film-flip FMR (FF-FMR)". In the "Stripline vs. Cavity (SVCFMR)" method the measurement in the flipped configuration is replaced (or complemented) with a FMR measurement taken with a microwave cavity.

If a film is uniform in both in-plane and perpendicular-to-plane directions, in the stripline FMR traces one expects to observe at least two modes: the fundamental mode and the $1^{\text {st }}$ SSWM. For symmetry reasons the amplitude of the $1^{\text {st }}$ SSWM with the respect of the fundamental mode amplitude should be the same for both flipped and unflipped configurations. However, as follows from Fig. 30(c) the $1^{\text {St }}$ SSWM will not be present at all in the cavity data.

Importantly, combining this method with the SVC-FMR protocol will allow one to distinguish between a perfectly thickness-uniform film and a film with a non-uniformity of magnetic parameters which is symmetric with respect to the film half-thickness plane. Indeed, in the latter case, one may expect the presence of the $2^{\text {nd }}$ SSWM in the cavity traces and of both $1^{\text {st }}$ and the $2^{\text {nd }}$ SSWM in the stripline data. Furthermore, the stripline response will not depend on the sample orientation in this case.

The SVC-FMR protocol alone works in a similar way. In this case, the measurement in the flipped configuration is replaced with a cavity measurement. Difference in the relative amplitude of the $1^{\text {st }}$ SSWM observed in these two measurements will evidence the presence of a thickness nonuniformity of the material. Furthermore, this method can be used to identify particular modes in the FMR spectra, when the spectra have complicated character, as it often happens for nano-patterned films.

Since both protocols rely on the observation of the exchange standing wave modes across the film thickness, the microwave frequency at which the data are taken should be high enough in order for the $1^{\text {st }}$ SSWM to exist for the magnetically saturated state of the material. For instance, for a $30 \mathrm{~nm}$-thick Permalloy film the $1^{\text {st }}$ SSWM emerges from the zero field at $\sim 18 \mathrm{GHz}$ if the magnetic field is applied in the sample plane. At $\sim 31 \mathrm{GHz}$ it will appear in the respective spectrum for a $20 \mathrm{~nm}$-thick Permalloy film and for a $17 \mathrm{~nm}$-thick film one will need to take measurements at $\sim 40 \mathrm{GHz}$ in order to observe this mode.

Below we overview several examples of applications of these two protocols.

\subsection{FF-FMR and SVC-FMR as tools with which to study magnetically stratified films and films with PMA}

The FF-FMR and SVC-FMR protocols as the methods for the investigation of magnetic nonuniformities in planar metallic ferromagnetic materials were proposed in Ref. [121]. A number of single-layer Permalloy films with thicknesses $40-110 \mathrm{~nm}$ were investigated with this method in that paper. Many of them did not show a signal of the $1^{\text {st }}$ SSWM in the unflipped orientation at all, which is not consistent with what is expected from the theory for a perfectly thickness uniform film [see Fig. 33(c)]. While flipped over, these films showed a noticeable response of this mode. 
Figure 34 shows data taken for a 100 nm-thick Permalloy film.

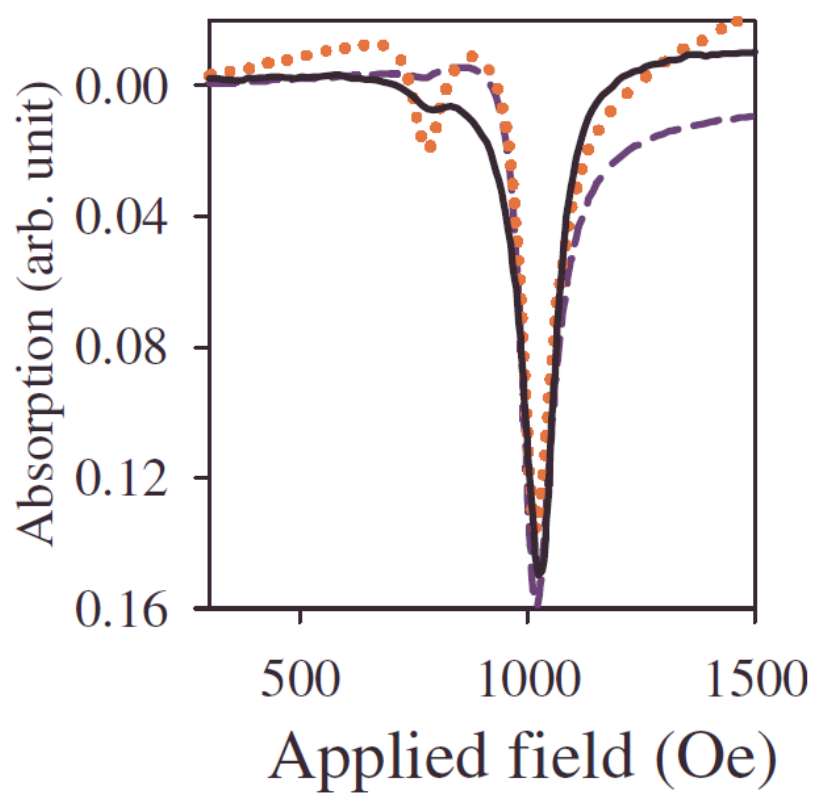

FIG. 34. Comparison of BFMR data with the cavity FMR data for a single-layer $100 \mathrm{~nm}$ Permalloy film grown on a sapphire substrate and having $10 \mathrm{~nm}$ thick Au capping and seed layers. Dashed line - film facing the broadband transducer. Dotted line - substrate facing the transducer. Solid line cavity FMR data. The graph shows anti-derivative of the raw data. Frequency $-9.527 \mathrm{GHz}$. Taken from [121].

From Fig. 34 one sees a signal of the $1^{\text {st }}$ SSWM in the traces taken with a cavity and with the stripline BFMR for the flipped film orientation. The difference in the relative amplitudes of this mode evidences that this film possesses some non-uniformity of its parameters in the direction of the film thickness. Potentially, this uniformity is located at one of the film surfaces, because of the strong differences in the responses for the flipped and unflipped orientations [compare Fig. 34 and Fig. 27].

This observation was explained in the framework of a model of the presence of a magnetically depleted layer at one of the film surfaces. By fitting the experimental data with the numerical model from [90] it was found that the respective magnetically depleted layer should be located at the film surface facing the external space (i.e. not at the film interface with the substrate). Otherwise, the amplitude of the $1^{\text {st }}$ SSWM would be larger for the unflipped orientation. Also, the thickness and the saturation magnetisation for the depleted layer were extracted from these fits ( $10 \mathrm{~nm}$ and $4 \pi M_{\mathrm{s}}=4000 \mathrm{G}$, respectively). Once a suitable model for the film structure has been found, the information delivered by the fits must be quite accurate, since one has to simultaneously fit not only the relative amplitudes of the modes for the two sample orientations, but also the resonant peak positions for all the observed modes.

Of course, the extracted information strongly relies on the model. Therefore, some independent information about the film structure would be beneficial for improving the accuracy of the prediction by this method. In the absence of such, an alternative model for the data in Fig. 34 is the presence of the PMA at one of the film surfaces. Figure 35 shows results of numerical 
simulations using the theory from [90] for a single-layer $30 \mathrm{~nm}$ Permalloy film with PMA. Frequency is $15 \mathrm{GHz}$.
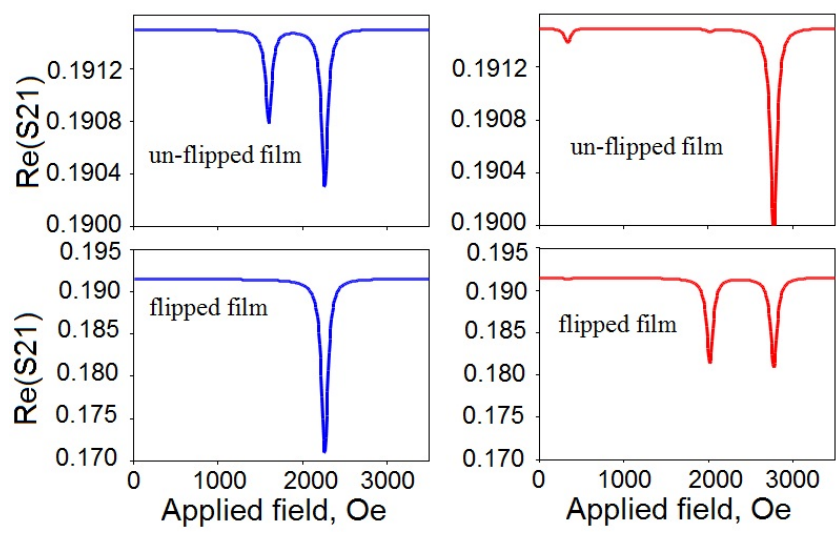

FIG. 35 Results of numerical simulations using the theory from [90] for a single-layer $30 \mathrm{~nm}$ Permalloy film with PMA. Frequency is $15 \mathrm{GHz}$. Left column: $K_{\mathrm{u}}<0$ (easy-plane surface anisotropy), right panel: $K_{\mathrm{u}}>0$ (easy axis anisotropy). PMA is present at the film surface facing the external space.

One sees that the spectra for the opposite signs of the surface anisotropy constant are the "mirror" images of each other: whereas for $K_{\mathrm{u}}<0$ one observes two peaks for the unflipped geometry and one peak for the flipped one, one will observe the opposite behaviour for $K_{\mathrm{u}}>0$. In this calculation, it is assumed that the PMA is present at the film surface facing the external space. Obviously, if the PMA is present at the film interface with the substrate, one will observe the opposite behaviour.

Thus, with this method it is possible either to determine the particular surface at which the surface anisotropy is present or the type of anisotropy (easy plane or easy axis). In order to determine both simultaneously, additional information is needed. One of the possible options is to look at the peak positions. One sees that the resonant fields for the easy-axis anisotropy are noticeably higher. This is because the easy-plane anisotropy results in the pinning of the dynamic magnetisation at the interface and the easy-axis one in "anti-" or negative pinning (Fig. 4).

The most reliable determination of the type of the surface anisotropy present in a film can be obtained if the anisotropy is repeatable from sample to sample. It is known that the impact of an interface anisotropy scales as $1 / L$ [194]. This is clearly seen from the simulation result in Fig. 36. As follows from this figure, the sign of the slope of the resonance frequency or the field dependence on $1 / L$ is determined by the type of anisotropy (easy-axis or easy-plane). This implies that the type of the anisotropy present in a sample may be determined from the sign of the slope of the dependence of the resonant field on the film thickness. The surface, at which the surface anisotropy is present, is then found from the FF-FMR data. 


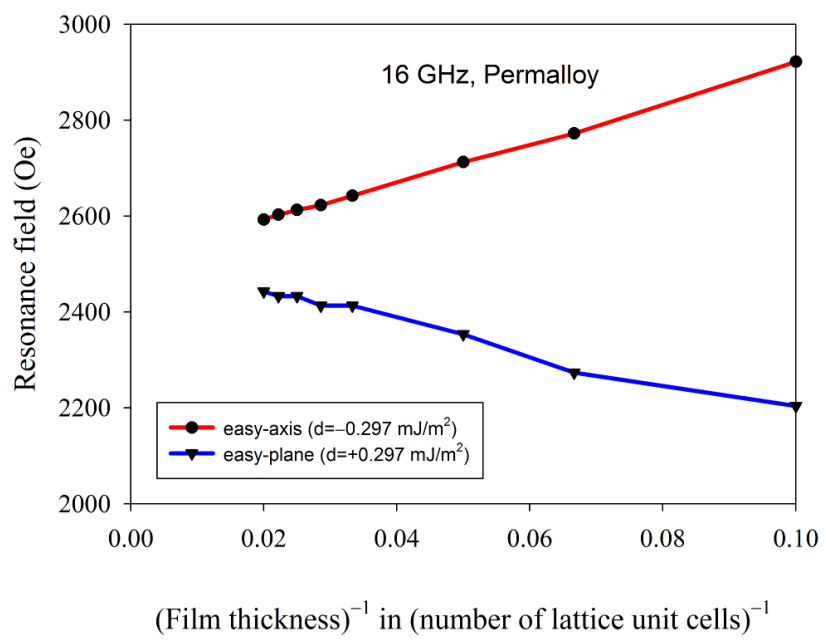

FIG 36. Simulated resonance field for a fundamental FMR mode in a single-layer Permalloy film as a function of the inverse film thickness $1 / L$.

As a final note to this discussion we mention that the traditional cavity FMR method is also able to determine if a film is thickness uniform or not. This is clearly seen from the solid line in Fig. 34. Unfortunately, for symmetry reasons this method fails to deliver any information about the location of this uniformity. The main advantage of the FF-FMR is its capability to determine how symmetric this uniformity with respect to the half-thickness plane of a metallic ferromagnetic film is. Furthermore, provided it is known which type of anisotropy to expect, the FF-FMR is able to deliver an answer to the question "Closer to which of the two film surfaces is the non-uniformity located?".

An alternative possibility to identify the type of the surface/interface anisotropy was recently confirmed with our film-flip FMR measurements of Si/Py/IrMn films [195]. This approach works when one knows the location of the surface/interface where this anisotropy is present. The $\mathrm{Si} / \mathrm{Py} / \mathrm{IrMn}$ films exhibit an exchange-bias effect [196, 197, 198, 199] at the interface of the ferromagnetic Permalloy and anti-ferromagnetic IrMn layers. The static field was applied in the film plane and along the exchange-bias direction. As shown in [77], physically the exchange bias is an in-plane uni-directional interface anisotropy leading to pinning of the dynamic magnetisation at the interface of the two layers. The experiments demonstrated that when the applied field was coaligned with the exchange-bias field direction, the FMR spectra were of the type shown in the right-hand column of Fig. 35. However, when the applied field was anti-aligned to the exchangebias field, the spectra taken in the flipped and unflipped orientations were of the type shown in the left-hand column of Fig. 35. The interface of the Permalloy layer, at which the interface unidirectional anisotropy is present, is precisely known for these materials. Consequently, from the FMR traces it is easy to infer that when the static field (and, consequently, the static magnetisation) is co-aligned with the exchange bias direction, a regime of the easy-axis-like interface anisotropy is implemented. In the case of the static magnetisation vector pointing to the direction opposite to the exchange bias direction, the interface conditions are, however, of the easy-plane like. (This plane is the film cross-section along the film thickness and perpendicular to the exchange-bias field.) Note that this experiment also delivers direct experimental evidence that the exchange bias is an interface effect. 


\subsection{SVC-FMR as a tool to interpret complicated FMR spectra}

It has been shown that the SVC-FMR protocol is a very useful tool which enables the interpretation of complicated FMR spectra. Indeed, FMR spectra may look very complex, especially for nano-patterned films. For these and similar materials it is always important to identify the standing-spin-wave modes, which give rise to particular absorption peaks seen in FMR spectra. Different selection rules for the mode excitation in the cavity FMR and stripline FMR experiments may help to carry out the mode identification.

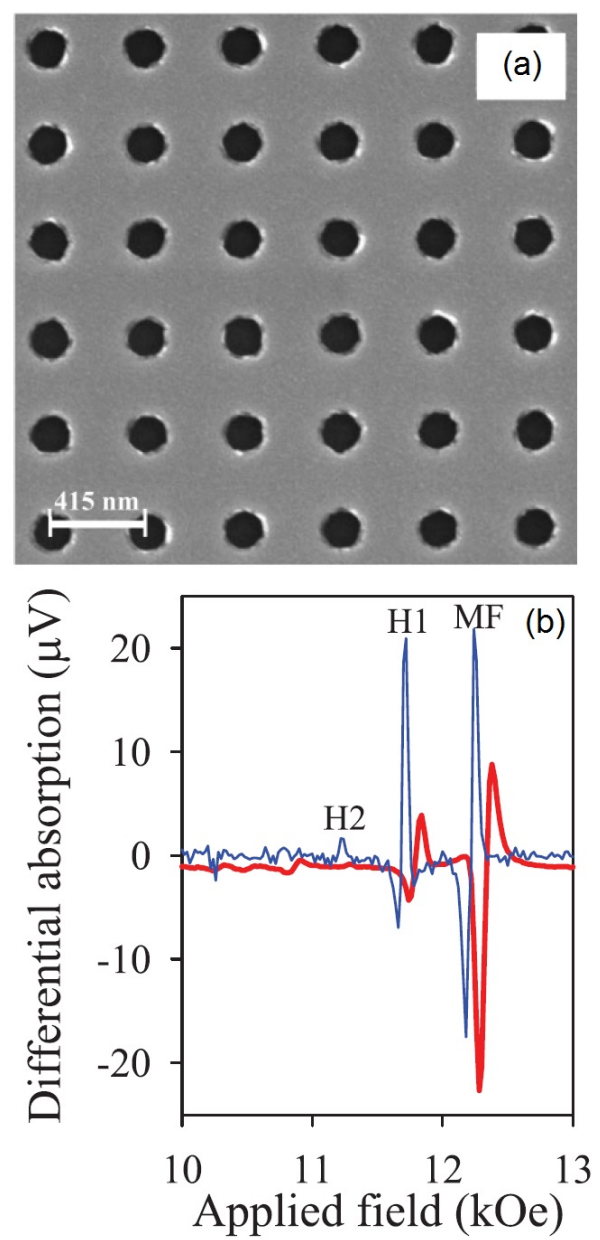

FIG. 37 (a) SEM image of the $80 \mathrm{~nm}$-thick anti-dot nanostructure made of Permalloy. (b) Experimental absorption traces for the nanostructure. Thin solid line: stripline BFMR at $9.5 \mathrm{GHz}$; thick solid line: cavity FMR at $9.529 \mathrm{GHz}$. From [184].

Figure 37(b) shows two FMR traces from Ref. [184]. These traces were registered for a $80 \mathrm{~nm}-$ thick anti-dot structure magnetised perpendicular to plane [Fig. 37(a)]. The trace shown by the thinner line was taken with a stripline BFMR and the thicker line is the result of a cavity FMR measurement. One sees that the mode in the stripline BFMR spectrum (denoted as H2) is completely missing in the cavity spectrum. The mode H1 displays significantly smaller relative amplitude (with respect to the largest peak in each respective spectrum) in the cavity data. This identifies the peaks $\mathrm{H} 1$ and $\mathrm{H} 2$ as thickness non-uniform modes which are counterparts of the $1^{\text {st }}$ SSWM of the parent continuous films. The peaks, which do not show a significant reduction in the amplitude in the cavity data, are then the responses of the modes which are standing spin waves in the sample plane formed on the basis of the fundamental mode (of uniform precession) of the parent 
continuous film.

\subsection{Effect of eddy-currents in non-magnetic metallic layers on the stripline FMR response of metallic multi-layered materials}

Combinations of metallic ferromagnetic and non-magnetic layers are building blocks for the nascent novel platform of electronics called spintronics [1, 33]. Many spintronic devices demonstrated so far operate at microwave frequencies and they are made of multi-layers consisting of non-magnetic metal (NM) and ferromagnetic metal (FM) thin films. A large amount of literature is devoted to the investigation of magnetisation dynamics and spin transport parameters of such multi-layers (e.g., [1, 14, 29, 34, 35, 41, 42, 47, 188, 200, 201, 202] to cite just a few recent articles). NM materials often used in these devices are $\mathrm{Pt}, \mathrm{Pd}, \mathrm{Cu}, \mathrm{Au}$, and $\mathrm{Ta}$.

The effect of the presence of NM capping and/or seed layers on the FMR response of the multi-layered films was investigated theoretically in Ref. [203]. A layered structure of the type used in the experimental paper [88] was considered. The model assumed that the stack contains 5 to $10 \mathrm{~nm}$-thin $\mathrm{Cu}$ or Ta seed and capping layers between which either a single Py layer or a pair of exchange coupled Co and Py layers is sandwiched.

Finite-Difference Time-Domain (FDTD) simulations were carried out to reveal magnetisation dynamics in this case. General information about the FDTD method can be found in Ref. [173]. The simulations predicted a crucial impact of the NM layers on the results of broadband FMR measurements of Co-Py bilayers. Figure 38 shows the simulated microwave absorption in the Co-Py bilayer from Ref. [88] without including the capping/seed layers into the simulation. In agreement with the experiment, one sees that the amplitude of the $1^{\text {st }}$ higher-order SSWM is larger than that of the fundamental mode. When one adds the Ta layers to the model, the amplitude of both fundamental (the first peak from the right) and $1^{\text {st }}$ higher-order SSWM increases. The gain in the amplitude of the fundamental mode is larger than that of the $1^{\text {st }}$ higher-order SSWM (the second peak from the right).

Dramatic changes in the response occur when $\mathrm{Ta}$ is replaced with $\mathrm{Cu}$ in the model. The conductivity of $\mathrm{Cu}$ is $5.95 \times 10^{7} \mathrm{~S} / \mathrm{m}$ that is almost eight times higher than the conductivity of Ta and three times higher than that of Co. The resonant absorption by the fundamental mode and the SSWMs increases and, most significantly, the intensity of the fundamental mode becomes larger than that of the $1^{\text {st }}$ higher-order SSWM (Fig. 38). 


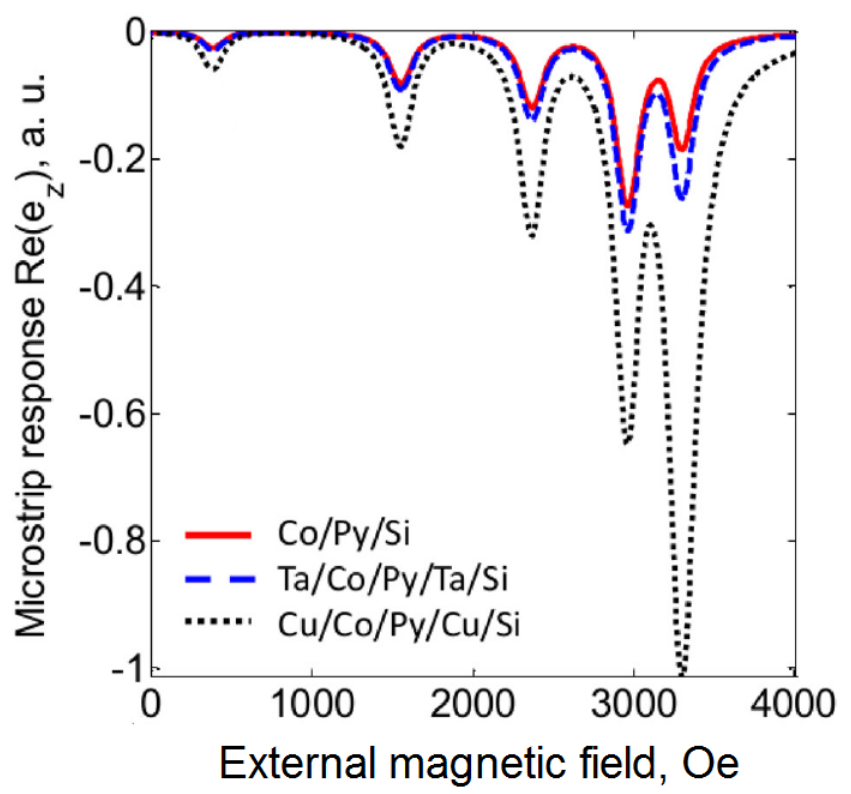

FIG. 38 FMR response of the $\mathrm{Co}-\mathrm{Permalloy}(\mathrm{Co} / \mathrm{Py})$ bi-layer structure with the stripline facing the Co side. Solid lines - no Ta layers, dashed lines - with $5 \mathrm{~nm}$ Ta layers from both sides of the Co/Py bilayer, and dotted lines $-5 \mathrm{~nm}$ of $\mathrm{Cu}$ is used instead of Ta. From [203].

The investigation of the distributions of the dynamic magnetic field across the film thickness helps to better understand the origin of the effect of the conductivity of the capping layer. As shown in Fig. 39, the magnetic field decreases through the Co-Permalloy bi-layer and vanishes at the rear surface of the Permalloy film. This result is in good agreement with the analytically derived boundary conditions Eq. (49) and numerical results in Fig. 32. We note that the slope of the magnetic field curve in Fig. 39 depends on the value of the conductivity: it is maximum in Co, because Co has a larger conductivity than Permalloy. However, the magnetic field profile becomes more homogeneous when the Co-Permalloy structure is sandwiched by the $\mathrm{Ta}$ or $\mathrm{Cu}$ layers. As shown in Fig. 39, the slope of the magnetic field curve decreases in Co and Permalloy layers in order to satisfy the condition that the field vanishes at the rear surface of the last metallic film. One also sees that the field slope increases as the conductivity of the film is increased. A decrease in the magnetic field slope (i.e., the field distribution becomes more homogeneous) explains why the intensity of the fundamental mode becomes higher than that of the $1^{\text {st }}$ higher-order SSWM when the Co-Py bilayer is surrounded by the two $\mathrm{Cu}$ films. 


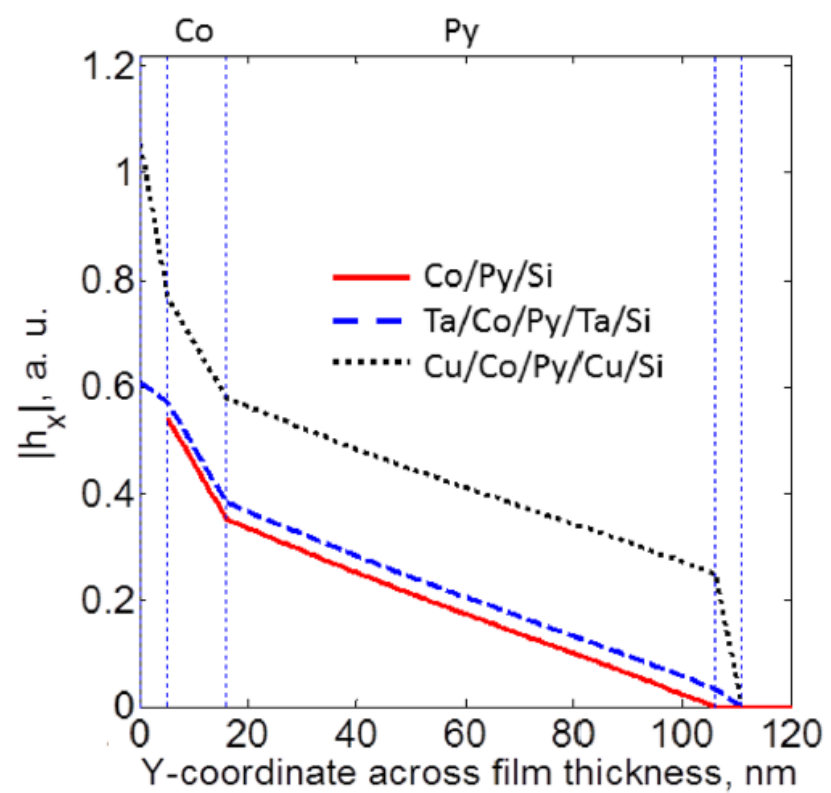

FIG. 39 In-plane dynamic magnetic field profiles across the thickness of Co-Permalloy bilayers. Frequency is $18 \mathrm{GHz}$. The profiles correspond to the $1^{\text {st }}$ higher-order SSWM. Solid lines - no Ta layers, dashed lines - with $5 \mathrm{~nm}$ Ta layers on both side of the Co/Py structure; dotted lines: $\mathrm{Cu}$ is used instead of Ta. The thin dashed lines denote the interface between the films. From [203].

Theoretical predictions made in Ref. [203] were confirmed experimentally by conducting BFMR measurements of $\mathrm{Cu}[d] / \mathrm{Ni}_{80} \mathrm{Fe}_{20}[70 \mathrm{~nm}] / \mathrm{Cu}[10 \mathrm{~nm}] / \mathrm{Si}$ multilayers, where $d$ is the thickness of the capping layer [172]. The samples are placed on top of a $0.33 \mathrm{~mm}$-wide microwave stripline (the capping layer side faces the stripline) and the FMR absorption is measured with a VNA. (We recall that in the case of a $0.33 \mathrm{~mm}$-wide microwave stripline the eddy current shielding should be very strong.) One sees that the capping layer strongly diminishes the absorption amplitude (Fig. 40). It quickly drops with an increase in the $\mathrm{Cu}$ capping layer thickness $d$. We stress that in this experiment $d<<\delta_{\mathrm{Cu}}$, where $\delta_{\mathrm{Cu}}$ is the classical skin depth of $\mathrm{Cu}$. This result has direct implications for the correct interpretation of raw broadband FMR data of highly conducting metallic multilayers and for measurements of spin current injection through interfaces implemented by placing a conducting multilayer on top of a microstrip or coplanar line.

Flovik et. al. [191] also experimentally investigated the effect of eddy currents on the FMR in FM-NM bilayers. Their experiments were conducted on Permalloy-Au and Permalloy-Cu samples, and they employed a cavity FMR setup. They demonstrated that the FMR response of the investigated bilayers is determined by the interaction between the microwave fields and the eddycurrent fields and that this interaction is tuneable through changing the sample geometry and the thickness of the NM layers. As the authors claim, the eddy currents were circulating along the edges of the rectangular sample. Indeed, as follows from Fig. 30, the eddy currents should be strongly suppressed in the bulk of the sample due to the symmetry of the microwave fields of the cavity. However, they may not be completely vanishing in the bulk due to the difference in conductivities for the two layers which breaks the symmetry. Similarly, the reason why the currents are present along the edges, is a break in translational symmetry which is introduced by the finite in-plane sample sizes. 


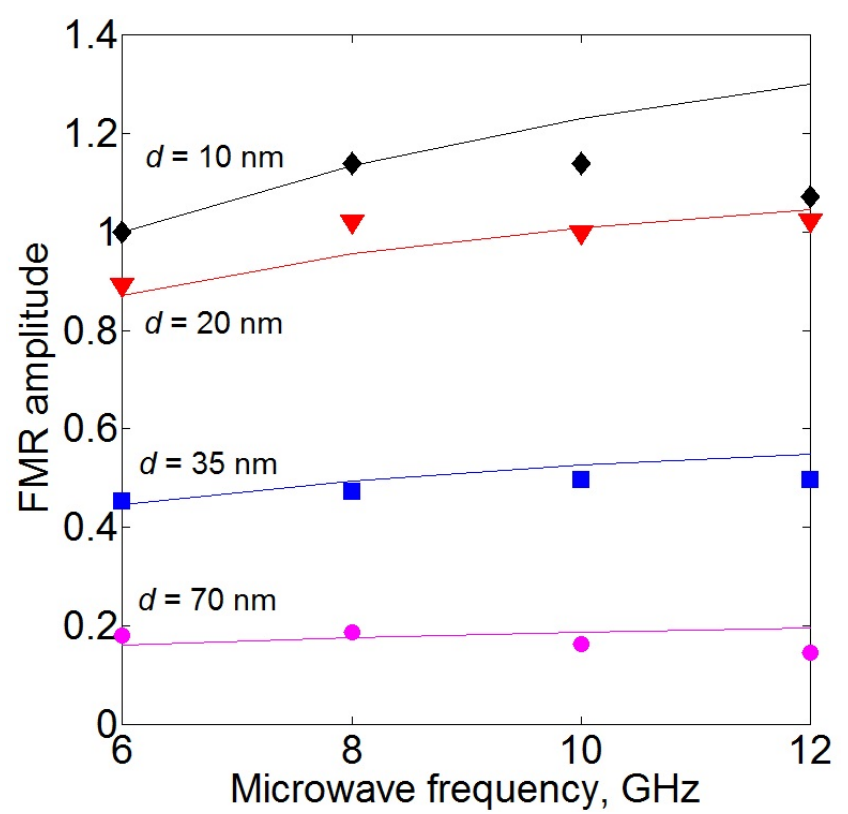

FIG. 40 Measured (symbols) and simulated (curves) amplitudes of the microstrip BFMR response for $\mathrm{Cu}[d] /$ Permalloy[70nm] $/ \mathrm{Cu}[10 \mathrm{~nm}] / \mathrm{Si}$ samples.

\subsection{Microwave eddy current contribution to the stripline FMR response of nano-patterned films}

Presently, a large number of experimental and theoretical works are concerned with measuring and understanding the FMR response of nano-patterned films (see, e.g., [1, 14, 16, 122, $184,204,205])$. One of the popular methods of FMR measurements of these materials is the stripline FMR (see, e.g., [106, 112, 120, 122, 184, 205, 206, 207, 208, 209, 210, 211]). It is natural to suppose that the microwave eddy-current effects due to the single-side incidence of microwave field also exist for these materials.

A trivial consequence of the single-surface microwave field incidence on plane ferromagnetic nanostructures has been already discussed in Section 7.2. We have shown that similar to continuous films the single-surface incidence leads to efficient excitation of standing spin wave modes across the material thickness (Fig. 37). However, the main goal of nano-patterning of ferromagnetic films is to make the magnetisation dynamics non-uniform in the material plane by introducing geometrical confinement in the plane. In this case, the resonant eigen-modes represent spin waves which are standing in the film plane. The geometrical confinement introduces an effective dipole magnetisation pinning at the edges of the nano-patterned structure [212]. Most often one is interested in the modes which are uniform in the direction of the material thickness, i.e. formed on the basis of the fundamental FMR mode of the parent continuous film.

The impact of microwave eddy currents on the FMR response of nanostructures has been recently demonstrated for model geometry which is very convenient for the studies of the effect of nano-confinement on the magnetisation dynamics. This geometry is a periodic array of long parallel metallic ferromagnetic nanostripes with a rectangular cross-section. The static magnetic field is applied along the stripes. This produces a single-domain state for each individual stripe with the static magnetisation vector pointing perfectly along the stripes all across the stripe cross-section [213]. Also, often the stripe size in the array plane ("stripe width" $w$ ) is significantly larger than its thickness $L(w>>L)$. This allows reducing the mathematical description of the magnetisation 
dynamics in this medium to a simple one-dimensional model [212].

In the experiment [120] a periodic array of Permalloy $4 \mathrm{~mm}$-long nanostripes was fabricated using a deep ultraviolet lithography followed by a lift-off process [214]. The fabricated stripes have the width $w=300 \mathrm{~nm}$. The edge-to-edge separation of the neighbouring elements is $d=115 \mathrm{~nm}$. The thickness of the elements is $h=25 \mathrm{~nm}$. BFMR measurements were taken by applying the static magnetic field along the stripe length. The width of the microstrip line used in measurements was $0.3 \mathrm{~mm}$.

Figure 41(a) shows a scanning electron micrograph of a typical magnetic nanostripe array. Figure 41(b) shows the position of the sample on top of a $0.3 \mathrm{~mm}$-wide microstrip line transducer used to take these measurements.

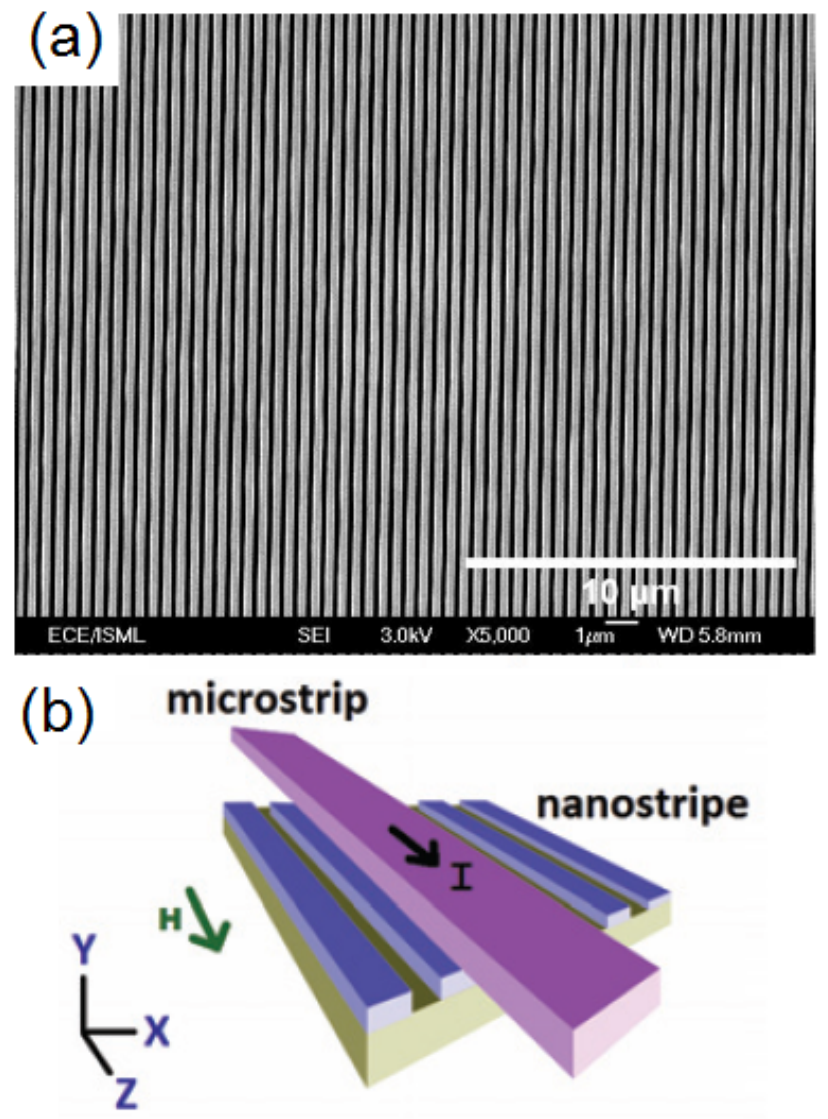

FIG. 41 (a) Scanning electron micrograph of a magnetic nanostripe array. (b) Sketch of microstrip BFMR arrangement with the stripline parallel to the applied field. Note that the microstrip is shown not to scale. From [86].

Nanostructuring of magnetic films strongly reduces the amplitude of the FMR response, which becomes comparable with or falls below the noise level. This makes the observation of the higher-order resonance modes difficult. Consequently, in order to increase the number of the higher-order modes seen in the experiment, the field modulation method is used in combination with a high-sensitivity FMR receiver (Section 2.5). Recall that in this case the registered FMR signal takes the form of the first derivative of the Lorentzian curve with respect to the parameter which is swept to register the absorption traces (so-called "differential absorption"). 
The result of measurements for the driving microwave frequency $15.004 \mathrm{GHz}$ is shown in Fig. 42(a) [120]. The trace taken with the standard sensitivity is shown by the solid line. One observes a strong fundamental mode (the highest frequency mode) and a number of small additional peaks at lower applied fields. The resonance modes in this case are spin waves with either odd $n=1,3,5, \ldots$ or even $(n=0,2,4, \ldots)$ nodes of the standing spin wave in the $x$-direction (see Fig. 3 in [120]). Consequently, the fundamental mode $(n=0)$ and two higher-order even modes $(n=2$ and $n=4$ ) have symmetric profiles and the profiles for the odd modes $n=1,3$, and 5 are anti-symmetric in the $x$-direction. The low-noise receiver allows taking measurements at a much higher sensitivity level of the lock-in amplifier [dashed line in Fig. 42(a)]. The signal of the fundamental mode is clipped in this case; however, up to five higher-order absorption peaks can easily be resolved.

For the symmetry reason only the even modes should be excited by the microwave magnetic field of the stripline. However, in Fig. 42(a) one notices at least three higher-order modes with very comparable amplitudes. Numerical simulations of the spectrum of eigen-modes identify the peaks 1,3 , and 5 as the anti-symmetric modes with the respective mode numbers.

The microwave magnetic field of the stripline is highly uniform on the length scale of the nanostripe width [see Fig. 7(a)]. This excludes a reason that the anti-symmetric modes are observed because of the spatial non-uniformity of the driving field. A second possible reason for the excitation of these modes is non-equal strengths of magnetisation pinning at the two lateral stripe edges. To exclude this possibility, in a separate experiment [215] the same sample was measured with the static magnetic field applied perpendicular to the sample plane. No signal of the antisymmetric modes was observed. Hence, this second experiment suggests that the magnetisation pinning at the lateral edges is negligible or its strength is the same at both stripe edges.

The only plausible explanation for the presence of the anti-symmetric modes in the FMR absorption spectrum is an effect of microwave eddy currents. As in the case of continuous films, the eddy currents in the nanostripes are excited by the microwave magnetic field of the stripline transducer. They flow in the direction opposite to the direction of the current in the stripline and hence along the nanostripes. In this direction the sample length is $4 \mathrm{~mm}$, which ensures the current continuity and hence the existence of the currents (see the discussion in the end of Section 5.2). Because the nanostripe cross-section is much smaller than the microwave skin depth, one may expect a uniform distribution of the eddy current density over the cross-section. The in-plane component of the Oersted field of the eddy-current is trivial: it has an asymmetric thickness profile of the type shown in the right-hand side panel of Fig. 21(b) along the stripe thickness $L$ (i.e. in the $y$ - direction) and a quasi-uniform and symmetric profile in the $x$-direction.

Because of this type of symmetry this component is unable to couple to the standing spin wave modes, which are anti-symmetric in the $x$-direction but uniform along the nanostripe thickness. Importantly, the film nano-patterning produces one more component of the Oersted field - the perpendicular-to-plane one. Its profile in the $x$-direction is shown in Fig. 43. One sees that it is perfectly anti-symmetric. This type of symmetry is ideal for the excitation of the anti-symmetric modes, provided the driving field couples efficiently to the dynamic magnetisation. When the static field is applied in the sample plane along the stripes, as in Fig. 41(b), the $x$-component of the eddycurrent field is perpendicular to the vector of the static magnetisation. Hence, it can contribute to the excitation of the magnetisation dynamics. However, if the static field is applied perpendicular to the sample plane, as in Ref. [215], this microwave-field component is along the vector of the static magnetisation and hence cannot couple to the dynamic magnetisation. 
This explains why the anti-symmetric modes were seen in [120], but not in [215]. Furthermore, the experiment from [120] is in very good agreement with numerical simulation (Fig. 41). In this simulation, it was assumed that a PP component with the profile from Fig. 43 is present in the driving microwave field. Importantly, it is easy to demonstrate that the PP component of the eddy-current field exists only because of the single-side incidence of the microwave field onto the nanostructure plane in the conditions of stripline FMR. In the case of the two-side incidence, as in a cavity FMR, the two partial eddy currents would cancel each other [see the sketch in Fig. 30(c)] and this field component would not exist.

As stated in Ref. [120], the strength of the excitation of the anti-symmetric modes by the eddy current field should be a function of the stripe magnetisation precession ellipticity $\varepsilon$ (see Sect. 4.2) and hence of the aspect ratio $p=w / L$. Indeed, in our recent work [215] we have observed a decrease in the amplitudes of the $n=1,3, \ldots$ modes with a decrease in $p$. This fact and the fact that the anti-symmetric modes are not seen in the PP FMR geometry represent a strong experimental evidence of the eddy-current contribution to the stripline FMR dynamics of the metallic ferromagnetic nanostripes. Obviously, if the direction of the material magnetisation is suitable, similar effects may be seen for other planar nanostructures, for which the continuity of the eddy currents may be ensured (e.g. rings, discs, anti-dots, etc.).

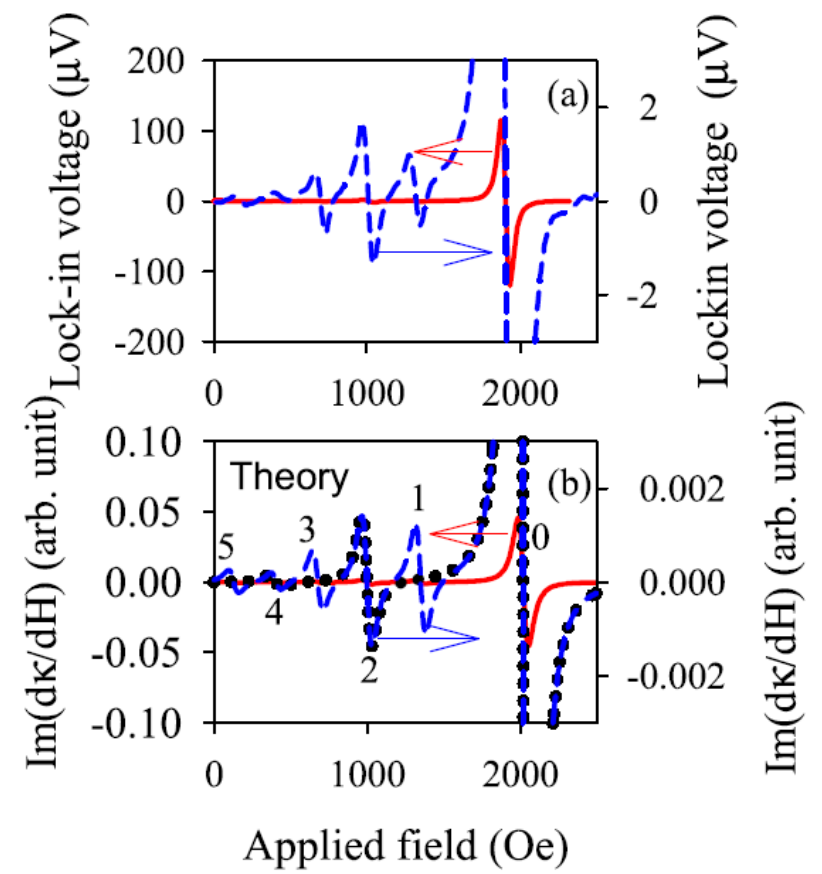

FIG. 42 Experimental (a) and theoretical (b) differential absorption traces for an array of parallel nanostripes made of Permalloy. Microwave frequency is $15.004 \mathrm{GHz}$. Solid lines - standard sensitivity/unzoomed, dashed lines - increased sensitivity/zoomed-in. Dotted line in (b) - result of simulation not taking into account the contribution of eddy currents to magnetisation dynamics. Numbers in (b) are the mode numbers identified by means of numerical simulations in [120]. From [120]. 


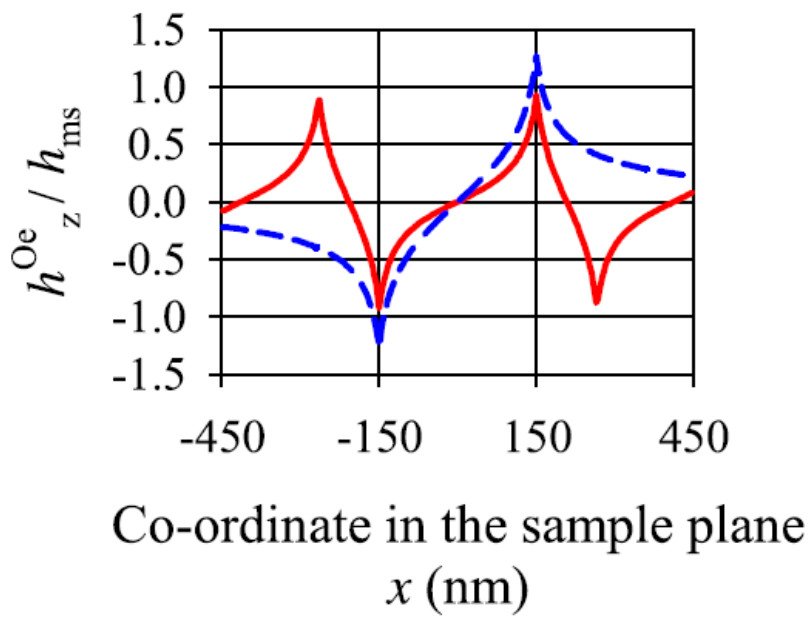

FIG. 43 The perpendicular-to-plane component of the microwave magnetic field of eddy currents induced inside the stripes. Dashed line: single nanostripe. Solid line: periodical array of parallel nanostripes. The stripe edges are located at $\pm 150 \mathrm{~nm}$. The sharp peaks of the solid line at $\pm 265 \mathrm{~nm}$ correspond to the nearest edges of the neighbouring stripes. From [120].

As a final note to this paragraph, we would like to mention that often broadband FMR measurements are taken on nanostructures lithographically formed on top of a signal line of a coplanar waveguide (see, e.g., [216]) or on top of a microstrip line (see, e.g., [217]). As we mentioned in Section 4.1, a decrease in the stripline width increases the microwave current density in the stripline which improves the FMR signal.

Also, as explained in Section 5.2.1 for striplines of microscopic width ( $w<30 \mu \mathrm{m}$ or so) the contribution of eddy-current shielding effect to the FMR amplitudes is negligible. This explains the fact that the eddy-current effects are usually not seen for the samples lithographically formed on top of microscopic striplines [216]. This observation suggests that the method of forming the nanostructures on top of a microscopic stripline may be very useful, when one wants to avoid the shielding effect and to simultaneously boost the FMR signal.

\section{Related topics}

Whereas we have endeavoured to introduce almost every aspect of the stripline BFMR, a few related areas could not be covered due to the large extent of this topic. For instance, we have not presented the areas of magnetically tuneable microwave metamaterials (see, e.g., $[53,54,218]$ ) and nonlinear metamaterials [219, 220], where the BFMR is also employed.

Also, often the stripline technique is used to drive magnetisation precession in ferromagnetic materials, but the FMR absorption signal is read in a "non-microwave" way, for instance with an MFM-tip ("MFM-FMR", see, e.g., [221, 222, 223, 224, 225, 226]), with micro-focus Brillouin Light Scattering (BLS) method (see, e.g., [227, 228]), or using the inverse Spin-Hall Effect (see, e.g., $[229,230,231,232])$ or similar electronic/spintronic methods (see, e.g., [86, 233, 234, 235, 236, 237]).

We also have not described the large areas of magnonics and spintronics in their entirety, but addressed these topics only from a perspective of BFMR as such. Spin caloritronics, a research 
direction recently highlighted in The 2014 Magnetism Roadmap [1], might also be mentioned in the context of the BFMR technique (see, e.g., [238]). Finally, we have not highlighted the emerging area of magnetic nanoparticles for biomedicine [239, 240, 241], also mentioned in The 2014 Magnetism Roadmap, where the BFMR is employed, too [55, 56, 57].

\section{Conclusions}

We have presented a critical overview of the rapid progress in the physics and applications of stripline BFMR since its emergence in the literature. Results from more than 240 articles, textbooks, and technical reports have been presented, and many practical examples discussed in detail. We believe that this review will be of interest to both general physical audience and specialists conducting research on various aspects of microwave nanomagnetism. It is clear that the research area of the BFMR spectroscopy is yet to reach its peak and to provide a valuable overall impact on the field of nanomagnetism.

\section{Acknowledgements}

This work has been supported by the Australian Research Council, the University of Western Australia (UWA), and UWA's Faculty of Science. ISM thanks UWA for a post-doctoral research fellowship (UPRF Scheme). We thank R. Magaraggia, K. Kennewell, N. Ross, C. Chang, L. Willig, C. Lueng, Z. Zhang, J. Hutomo, X. Wu, P. Metaxas, R. Stamps, A. Adeyeye, A. Stashkevich, and R. Bali for their valuable input. Software used to obtain some of the numerical results presented in this article can be downloaded for free from the web-site of the Spintronics and Magnetisation Dynamics group of the University of Western Australia (http://www.physics.uwa.edu.au/research/spindynamics), or by contacting the corresponding author (M.K.).

Appendix I - Magnetic units conversion factors

\begin{tabular}{|c|c|c|c|c|}
\hline & Symbol & Gaussian \& cgs emu & $\begin{array}{c}\text { Conversion } \\
\text { factor, } \mathrm{C}^{\mathrm{b}}\end{array}$ & $\begin{array}{c}\text { SI \& rationalised } \\
\mathrm{mks}\end{array}$ \\
\hline $\begin{array}{c}\text { Magnetic flux density, } \\
\text { magnetic induction }\end{array}$ & $\mathrm{B}$ & Gauss $(\mathrm{G})$ & $10^{-4}$ & Tesla $(\mathrm{T}), \mathrm{Wb} / \mathrm{m}^{2}$ \\
\hline Magnetic field strength & $\mathrm{H}$ & Oersted $(\mathrm{Oe})$ & $10^{3} /(4 \pi)$ & $\mathrm{A} / \mathrm{m}$ \\
\hline Magnetisation & $4 \pi \mathrm{M}_{\mathrm{s}}$ & $\mathrm{G}$ & $10^{3} /(4 \pi)$ & $\mathrm{A} / \mathrm{m}$ \\
\hline Permeability & $\mu$ & dimensionless & $4 \pi \times 10^{-7}$ & $\mathrm{H} / \mathrm{m}, \mathrm{Wb} /(\mathrm{A} \cdot \mathrm{m})$ \\
\hline Demagnetisation factor & $\mathrm{N}$ & dimensionless & $1 /(4 \pi)$ & $\mathrm{dimensionless}$ \\
\hline $\begin{array}{c}\text { Interlayer exchange } \\
\text { constant }\end{array}$ & $\mathrm{A}_{12}$ & erg $/ \mathrm{cm}^{2}$ & $10^{-7}$ & $\mathrm{~J} / \mathrm{m}^{2}$ \\
\hline
\end{tabular}

${ }^{\mathrm{a}}$ Gaussian units and cgs emu are the same for magnetic properties. The defining relation is $\mathbf{B}=\mathbf{H}+4 \pi \mathbf{M}$.

${ }^{b}$ Multiply a number in Gaussian units by $\mathrm{C}$ to convert it to SI. 


\section{References}

[1] R. L. Stamps, S. Breitkreutz, J. Åkerman, A. V. Chumak, Y.-C. Otani, G. E W Bauer, J.-U. Thiele, M. Bowen, S. A. Majetich, M. Kläui, I. L. Prejbeanu, B. Dieny, N. M. Dempsey, and B. Hillebrands, J. Phys. D: Appl. Phys. 47, 333001 (2014).

[2] "Japanese satellite first to use magnetic memory", Scientific American, 13 March 2008, http://www.scientificamerican.com/article.cfm?id=japanese-satellite-mram-freescale.

[3] J. H. E. Griffiths, Nature, 158, 670 (1946).

[4] C. Kittel, Phys. Rev. 71, 270 (1947).

[5] C. Kittel, Phys. Rev. 73, 155 (1948).

[6] C. Kittel and C. Herring, Phys. Rev. 77, 725 (1950).

[7] L. R. Walker, Phys. Rev. 105, 390 (1957).

[8] J. R. Eshbach and R. W. Damon, Phys. Rev. 118, 1208 (1960).

[9] R. W. Damon and J. R. Eshbach, J. Phys. Chem. Solids 19, 308 (1961).

[10] C. E. Patton, Phys. Rep. 103, 251 (1984).

[11] N. Cramer, D. Lucic, R. E. Camley, and Z. Celinski, J. Appl. Phys. 87, 6911 (2000).

[12] V. Vlaminck and M. Bailleul, Science 322, 410 (2008).

[13] T. J. Fal, V. Veerakumar, B. Kuanr, Y. V. Khivintsev, Z. Celinski, and R. E. Camley, J. Appl. Phys. 102, 063907 (2007).

[14] V. V. Kruglyak, S. O. Demokritov, and D. Grundler, J. Phys. D: Appl. Phys. 43, 264001 (2010).

[15] A. A. Serga, A. V. Chumak, and B. Hillebrands, J. Phys. D: Appl. Phys. 43, 264002 (2010).

[16] B. Lenk, H. Ulrichs, F. Garbs, M. Münzenberg, Phys. Rep. 507, 107 (2011).

[17] M. P. Kostylev, A. A. Serga, T. Schneider, B. Leven, and B. Hillebrands, Appl. Phys. Lett. 87, 153501 (2005).

[18] T. Schneider, A. A. Serga, B. Leven, B. Hillebrands, R. L. Stamps, and M. P. Kostylev, Appl. Phys. Lett. 92, 022505 (2008).

[19] K.-S. Lee and S.-K. Kim, J. Appl. Phys. 104, 053909 (2008).

[20] A. Khitun, M. Bao, and K. L. Wang, J. Phys. D: Appl. Phys. 43, 264005 (2010).

[21] Z. Gu, M. E. Nowakowski, D. B. Carlton, R. Storz, M.-Y. Im, J. Hong, W. Chao, B. Lambson, P. Bennett, M. T. Alam, M. A. Marcus, A. Doran, A. Young, A. Scholl, P. Fischer, J. Bokor, arXiv:1408.3157 (2014).

[22] The Physics of Ultrahigh-Density Magnetic Recording, ed. M. Plumer, J. van Ek, and D. Weller. Springer, Berlin, 2001.

[23] J. M. Slaughter, R. W. Dave, M. DeHerrera, M. Durlam, B. N. Engel, J. Janesky, N. D. Rizzo, and S. Tehrani, J. Superconductivity 15, 19 (2002).

[24] S. Tehrani, J. M. Slaughter, M. DeHerrera, B. N. Engel, N. D. Rizzo, J. Salter, M. Durlam, R. W. Dave, J. Janesky, B. Butcher, K. Smith, and G. Grynkewich, Proc. IEEE 91, 703 (2003).

[25] A. Ney, C. Pampuch, R. Koch, and K. H. Ploog, Nature 425, 485 (2003).

[26] B. D. Terris, J. Magn. Magn. Mater. 321, 512 (2009).

[27] M. V. Costache, M. Sladkov, S. M. Watts, C. H. van der Wal, and B. J. van Wees, Phys. Rev. Lett. 97, 216603 (2006).

[28] K. Hika, L.V. Panina, and K. Mohri, IEEE Trans. Magnet. 32, 4594 (1996).

[29] C. S. Chang, M. Kostylev, and E. Ivanov, Appl. Phys. Lett. 102, 142405 (2013).

[30] F. Klose, Ch. Rehm, D. Nagengast, H. Maletta, and A. Weidinger, Phys. Rev. Lett. 78, 1150 (1997).

[31] K. Munbodh, F. A. Perez, and D. Lederman, J. Appl. Phys. 111, 123919 (2012).

[32] J. C. Slonczewski, J. Magn. Magn. Mater. 159, L1 (1996). 
[33] A. Hirohata and K. Takanashi, J. Phys. D: Appl. Phys. 47, 193001 (2014).

[34] A. Brataas, A. D. Kent, and H. Ohno, Nat. Mater. 11, 372 (2012).

[35] T. Jungwirth, J. Wunderlich, and K. Olejník, Nature Mater. 11, 382 (2012)

[36] M. Ranjbar, F. Gerhard, P. Dürrenfeld, R. K. Dumas, M. Balinsky, C. Gould, L. W. Molenkamp, and J. Akerman, "Spin Pumping in NiMnSb/Pt bilayers", abstract of the ICMM 2014 Conference, Sendai, Japan, 29 June- 2 July 2014.

[37] O. d'Allivy Kelly, A. Anane, R. Bernard, J. Ben Youssef, C. Hahn, A H. Molpeceres, C. Carrétéro, E. Jacquet, C. Deranlot, P. Bortolotti, R. Lebourgeois, J.-C. Mage, G. de Loubens, O. Klein, V. Cros, and A. Fert, Appl. Phys. Lett. 103, 082408 (2013).

[38] H. Chang, P. Li, W. Zhang, T. Liu, A. Hoffmann, L. Deng, and M. Wu, IEEE Magnetics Lett. 5, 6700104 (2014).

[39] M. Prutton, Thin Ferromagnetic Films (Butterworth, Washington, 1964).

[40] J. M. H. Seavey and P. Tannenwald, Phys. Rev. Lett. 1, 168 (1958).

[41] J. M. Shaw, H. T. Nembach, and T. J. Silva, Phys. Rev. B 87, 054416 (2013).

[42] C. T. Boone, H. T. Nembach, J. M. Shaw, and T. J. Silva, J. Appl. Phys. 113, 153906 (2013).

[43] D. D. Stancil and A. Prabhakar, Spin Waves: Theory and Application, Springer, Berlin, 2009.

[44] R. Arias and D. L. Mills, Phys. Rev. B 60, 7395 (1999).

[45] K. Lenz, H. Wende, W. Kuch, K. Baberschke, K. Nagy, and A. Janossy, Phys. Rev. B 73, 144424 (2006).

[46] C. Bilzer, T. Devolder, P. Crozat, C. Chappert, S. Cardoso, and P. P. Freitas, J. Appl. Phys. 101, 074505 (2007).

[47] M. Weiler, J. M. Shaw, H. T. Nembach, and T. J. Silva, ArXiv:1409.1290v1.

[48] J. A. Katine, F. J. Albert, R. A. Buhrmann, E. B. Myers, and D. C. Ralph, Phys. Rev. Lett. 84, 3149 (2000).

[49] H. Morise and S. Nakamura, Phys. Rev. B 71, 014439 (2005).

[50] J. Grollier, V. Cros, H. Jaffrès, A. Hamzic, J. M. George, G. Faini, J. Ben Youssef, H. Le Gall, and A. Fert, Phys. Rev. B 67, 174402 (2003).

[51] Q. Mistral, A. Deac, J. Grollier, O. Redon, Y. Liu, M. Li, P. Wang, B. Dieny, and T. Devolder, Mater. Sci. Eng. B 126, 267 (2006).

[52] F. Falcone, F. Martín, J. Bonache, R. Marqués, and M. Sorolla, Microw. Opt. Technol. Lett. 40, 3 (2004).

[53] G. B. G. Stenning, G. J. Bowden, L. C. Maple, S. A. Gregory, A. Sposito, R. E. Eason, N. I. Zheludev, and P. A. J. de Groot, Opt. Express 21, 1456 (2013).

[54] S. A. Gregory, G. B. G. Stenning, G. J. Bowden, N. I. Zheludev, and P. A. J. de Groot, New. J. Phys. 16, 063002 (2014).

[55] S. Ghionea, P. Dhagat, and A. Jander, IEEE Sensors J. 8, 896 (2008).

[56] E. Chatterjee, T. Marr, P. Dhagat, and V. T. Remcho, Sensors and Actuators B 156, 651 (2011).

[57] P. J. Metaxas, M. Sushruth, R. Begley, J. Ding, M. Albert, W. Wang, H. Fangohr, R. C. Woodward, I. S. Maksymov, A.O. Adeyeye, and M. Kostylev, submitted

[58] B. Heinrich, Radio Frequency Techniques, in: B. Heinrich, J. Bland (Eds.), Ultrathin Magnetic Structures II: Measurement Techniques and Novel Magnetic Properties, Springer, 2005, pp. 195296.

[59] M. Farle, Rep. Prog. Phys. 61, 755 (1998).

[60] I. Neudecker, G. Woltersdorf, B. Heinrich, T. Okuno, G. Gubbiotti, C.H. Back, J. Magn. Magn. Mater. 307, 148 (2006). 
[61] P. Janssen, Technical report, University of Alabama, Center for Materials for Information Technology, Department of Physics and Astronomy, http://www.bama.ua.edu/ tmewes/People/Report\%20Paul\%20Janssen.pdf

[62] R. E. Camley, Z. Celinski, T. Fal, A. V. Glushchenko, A. J. Hutchison, Y. Khivintsev, B. Kuanr, I. R. Harward, V. Veerakumar, and V. V. Zagorodnii, J. Magn. Magn. Mater. 321, 2048 (2009).

[63] E. Montoya, T. McKinnon, A. Zamani, E. Girt, and B. Heinrich, J. Magn. Magn. Mater. 356, 12 (2014).

[64] O. Yalein, Ferromagnetic Resonance: Theory and Application, InTech Publishing, Zagreb, Croatia, 2013.

[65] G. S. Abo , Y.-K. Hong, J. Park , J. Lee , W. Lee , and B.-C. Choi. IEEE Trans. Magnet. 49, 4937, (2013).

[66] V. K. Arkad'yev, Absorption of electric waves in parallel wires, Journal of Russian Society of Physical Chemistry Society (Physics Series) 44, 165 (1912).

[67] J. Dorfmann, Z. Phys. 17, 98 (1923).

[68] C. Kittel, Phys. Rev. 110, 1295 (1958).

[69] C. Kittel, Introduction to Solid State Physics, $8^{\text {th }}$ ed. Wiley, New York, 2004.

[70] R. F. Soohoo, Magnetic Thin Films, Harper \& Row, New York, 1965.

[71] G. T. Rado and J. R. Weertman, J. Phys. Chem. Solids 11, 315 (1959).

[72] F. Hoffmann, Phys. Status Solidi B 41, 807 (1970).

[73] M. Vohl, J. Barnaś, and P. Grünberg, Phys. Rev. B 39, 12003 (1989).

[74] J. Barnaś, J. Magn. Magn. Mater. 102, 319 (1991).

[75] S.Krupicka, Physik der Ferrite und der Verwandten Magnetischen Oxide, Springer Fachmedien: Wiesbaden, 1973.

[76] B A Kalinikos, M P Kostylev, N V Kozhus and A N Slavin, J. Phys. Condens. Matter 2, 9861-9877 (1990).

[77] R. Magaraggia, K. Kennewell, M. Kostylev, R. L. Stamps, M. Ali, D. Greig, B. J. Hickey, and C. H. Marrows, Phys. Rev. B. 83, 054405 (2011).

[78] I. E. Dzialoshinskii, Sov. Phys. JETP 5, 1259 (1957).

[79] I. Dzyaloshinsky, J. Phys. Chem. Solids 4, 241 (1958).

[80] T. Moriya, Phys. Rev. Lett. 4, 228 (1960).

[81] T. Moriya, Phys. Rev. 120, 91 (1960).

[82] M. Kostylev, J. Appl. Phys. 115, 23 (2014).

[83] G. R. Eaton, S. S. Eaton, D. P. Barr, and R. T. Weber, Quantitative EPR, Springer-Verlag, New York, 2010.

[84] Y. Tabuchi, S. Ishino, T. Ishikawa, R. Yamazaki, K. Usami, and Y. Nakamura, Phys. Rev. Lett. 113, 083603 (2014).

[85] M. Goryachev, W. G. Farr, D. L. Creedon,1 Y. Fan, M. Kostylev, and M. E. Tobar, Phys. Rev. Appl. 2, 054002 (2014).

[86] C. S. Chang, M. Kostylev, A. O. Adeyeye, M. Bailleul, and S. Samarin, Euro. Phys. Lett. 96, 57007 (2011).

[87] S. Pinon, D. L. Diedhiou, A.-M. Gue, N. Fabre, G. Prigent, V. Conedera, E. Rius, C. Quendo, B. Potelon, J.-F. Favennec, and A. Boukabache, J. Micromech. Microeng. 22, 074005 (2012).

[88] K. J. Kennewell, M. Kostylev, N. Ross, R. Magaraggia, R. L. Stamps, M. Ali, A. A. Stashkevich, D. Greig, and B. J. Hickey, J. Appl. Phys. 108, 073917 (2010)

[89] T. Schneider, A. A. Serga, T. Neumann, B. Hillebrands, and M. P. Kostylev, Phys. Rev. B 77, 214411 (2008). 
[90] M. Kostylev, J. Appl. Phys. 106, 043903 (2009).

[91] P. Silvester, Proc. IEEE 115, 43 (1968).

[92] T. J. Silva, C. S. Lee, T. M. Crawford, and C. T. Rogers, J. Appl. Phys. 85, 7849 (1999).

[93] A. Abragam. Principles of Nuclear Magnetic Resonance, Cambridge University Press: Cambridge, UK, 1968.

[94] K. J. Kennewell, D. C. Crew, M. J. Lwin, R. C. Woodward, S. Prasad, and R. L. Stamps, Surface Science 601, 5766 (2007).

[95] A. Fessant, J. Geraltowski, J. Loaec, and H. Legall, J. Magn. Magn. Mater. 83, 557 (1990).

[96] G. Counil, J.-V. Kim, T. Devolder, C. Chappert, K. Shigeto, and Y. Otani, J. Appl. Phys. 95, 5646 (2004).

[97] G. Counil, P. Crozat, T. Devolder, C. Chapper, S. Zoll, and R. Fournel, IEEE Trans. Magn. 42, 3321 (2006).

[98] M. L. Schneider, T. Gerrits, A. B. Kos, and T. J. Silva, Appl. Phys. Lett. 87, 072509 (2005).

[99] S. S. Kalarickal, P. Krivosik, M. Wu, C. E. Patton, M. L. Schneider, P. Kabos, T. J. Silva, and J. P. Nibarger, J. Appl. Phys. 99, 093909 (2006).

[100] M. L. Schneider, A. B. Kos, and T. J. Silva, Appl. Phys. Lett. 85, 254 (2004).

[101] M. Belmeguenai, T. Martin, G. Woltersdorf, M. Maier, and G. Bayreuther, Phys. Rev. B 76, 104414 (2007).

[102] M. Belmeguenai, T. Martin, G. Woltersdorf, G. Bayreuther, V. Baltz, A. K. Suszka, and B. J. Hickey, J. Phys.: Condens. Matter 20, 345206 (2008).

[103] M. Belmeguenai, F. Zighem, Y. Roussigné, S.-M. Chérif, P. Moch, K. Westerholt, G. Woltersdorf, and G. Bayreuther, Phys. Rev. B 79, 024419 (2009).

[104] C. Brosseau, S. Mallégol, P. Quéffelec, and J. Ben Youssef, Phys. Rev. B 70, 092401 (2004).

[105] Y. Nozaki, K. Tateishi, S.-I. Taharazako, S. Yoshimura, and K. Matsuyama, J. Appl. Phys. 105, 013911 (2009).

[106] F. G. Aliev, J. F. Sierra, A. A. Awad, G. N. Kakazei, D.-S. Han, S.-K. Kim, V. Metlushko, B. Ilic, and K. Y. Guslienko, Phys. Rev. B 79, 174433 (2009).

[107] D. Crew, K. Kennewell, M. Lwin, R. Woodward, S. Prasad, and R. Stamps, J. Appl. Phys. 97, 10A707 (2005).

[108] M. Kostylev, R. Magaraggia, F. Y. Ogrin, E. Sirotkin, V. F. Mescheryakov, N. Ross, and R. L. Stamps, IEEE Trans. Magn. 44, 2741 (2008).

[109] V. K. Sakharov, Y. V. Khivintsev, S. A. Nikitov, and Y. A. Filimonov, FMR investigation of magnonic crystals based on cobalt and Permalloy. International conference "Days on Diffraction 2012", Saint Petersburg, May 28 - June 1, 2012. - P. 166-167.

[110] V. Vlaminck, J. E. Pearson, S. D. Bader, and A. Hoffmann, Phys. Rev. B 88, 064414 (2013).

[111] S. Neusser and D. Grundler, Adv. Mater. 21, 2927 (2009).

[112] J. F. Sierra, A. A. Awad, G. N. Kakazei, F. J. Palomares, and F. G. Aliev, IEEE Trans. Magnet. 44, 3063 (2008).

[113] J. Ding, S. Jain, and A. O. Adeyeye, J. Appl. Phys. 109, 07D301 (2011).

[114] J. Ding, D. Tripathy and A. O. Adeyeye, EPL 98, 16004 (2012).

[115] H. Głowiński, M. Schmidt, I. Gościańska, J-Ph. Ansermet, and J. Dubowik, J. Appl. Phys. 116, 053901 (2014).

[116] K. Kurokawa, IEEE Trans. Microwave Theory. Tech. 13, 194 (1965).

[117] S. Beguhn, Ziyao Zhou, S. Rand, X. Yang, J. Lou, and N. X. Sun, J. Appl. Phys. 111, 07 A503 (2012).

[118] H. Le Gall and N. Vukadinovic, IEEE Trans Magn. MAG-24, 3051 (1988).

[119] P. Dutta and P. M. Horn, Rev. Mod. Phys. 53, 497 (1981). 
[120] M. Kostylev, J. Ding, E. Ivanov, S. Samarin, and A. O. Adeyeye, J. Appl. Phys. 115, 173903 (2014).

[121] M. Kostylev, A. A. Stashkevich, A. O. Adeyeye, C. Shakespeare, N. Kostylev, N. Ross, K. Kennewell, R. Magaraggia, Y. Roussigné, and R. L. Stamps, J. Appl. Phys. 108, 103914 (2010).

[122] S. Jain, M. Kostylev, and A. O. Adeyeye, Phys. Rev. B 82, 214422 (2010).

[123] E. N. Ivanov and M. Kostylev, arXiv:1402.3459 (2014).

[124] S. Mangin, D. Ravelosona, J. A. Katine, M. J. Carey, B. D. Terris, and E. E. Fullerton, Nat. Mater. 5, 210 (2006).

[125] W. H. Rippard, A. M. Deac, M. R. Pufall, J. M. Shaw, M. W. Keller, S. E. Russek, G. E. W. Bauer, and C. Serpico, Phys, Rev. B 81, 014426 (2010)

[126] B. D. Terris and T. Thomson, J. Phys. D 38, R199 (2005)

[127] G. H. O. Daalderop, P. J. Kelly, and F. J. A. den Broeder, Phys. Rev. Lett. 68, 682 (1992).

[128] J. M. Shaw, H. T. Nembach, and T. J. Silva, Appl. Phys. Lett. 99, 012503 (2011).

[129] S. Hashimoto,Y. Ochiai, and K. Aso, J. Appl. Phys. 66, 4909 (1989).

[130] T. Suzuki, H. Notarys, D. C. Dobbertin, C.-J. Lin, D. Weller, D. C. Miller, and G. Gorman, IEEE Trans. Magn. 28, 2754 (1992).

[131] W. B. Zeper, H. W. Vankesteren, B. A. J. Jacobs, J. H. M. Spruit, and P. F. Carcia, J. Appl. Phys. 70, 2264 (1991).

[132] R. L. Stamps, L. Louail, M. Hehn, M. Gester, and K. Ounadjela, J. Appl. Phys. 81, 4751 (1997).

[133]. N. Engel, C. D. England, R. A. VanLeeuwen, M. H. Wiedmann, and C. M. Falco, Phys. Rev. Lett. 67, 1910 (1991).

[134] R. Law, R. Sbiaa, T. Liew, and T. C. Chong, Appl. Phys. Lett. 91, 242504 (2007).

[135] S. Sindhu, M. A. M. Haast, K. Ramstöck, L. Abelmann, and J. C. Lodder, J. Magn. Magn. Mater. 238, 246 (2002).

[136] S. Mangin, Y. Henry, D. Ravelosona, J. A. Katine, and E. E. Fullerton, Appl. Phys. Lett. 94, 012502 (2009).

[137] J. M. Shaw, M. Olsen, J. W. Lau, M. L. Schneider, T. J. Silva, O. Hellwig, E. Dobisz, and B. D. Terris, Phys. Rev. B 82, 144437 (2010).

[138] H. T. Nembach, T. J. Silva, J. M. Shaw, M. L. Schneider, M. J. Carey, S. Maat, and J. R. Childress, Phys. Rev. B 84, 054424 (2011).

[139] J. M. Shaw, H. T. Nembach, and T. J. Silva, Phys. Rev. B 85, 054412 (2012).

[140] L. Néel, J. Phys. Radium 15, 225 (1954)

[141] N. Nakajima, T. Koide, T. Shidara, H. Miyauchi, H. Fukutani, A. Fujimori, K. Iio, T. Katayama, M. Nývlt, and Y. Suzuki, Phys. Rev. Lett. 81, 5229 (1998).

[142] M. Tischer, O. Hjortstam, D. Arvanitis, J. H. Dunn, F. May, K. Baberschke, J. Trygg, J. M. Wills, B. Johansson, and O. Eriksson, Phys. Rev. Lett. 75, 1602 (1995).

[143] A. Hahlin, J. H. Dunn, O. Karis, P. Poulopoulos, R. Nünthel, J. Lindner, and D. Arvanitis, J. Phys.: Condens. Matter 15, S573 (2003).

[144] H. A.Dürr, G. van der Laan, J. Vogel, G. Panaccione, N. B. Brookes, E. Dudzik, and R. McGrath, Phys. Rev. B 58, R11853 (1998).

[145] W. Kuch, J. Gilles, S. S. Kang, S. Imada, S. Suga, and J. Kirschner, Phys. Rev. B 62, 3824 (2000).

[146] P. Ryan, R. P. Winarski, D. J. Keavney, J. W. Freeland, R. A. Rosenberg, S. Park, and C. M. Falco, Phys. Rev. B 69, 054416 (2004).

[147] B. T. Thole, P. Carra, F. Sette, and G. van der Laan, Phys. Rev. Lett. 68, 1943 (1992). 
[148] C. T. Chen, Y. U. Idzerda, H.-J. Lin, N. V. Smith, G. Meigs, E. Chaban, G. H. Ho, E. Pellegrin, and F. Sette, Phys. Rev. Lett. 75, 152 (1995).

[149] A. N. Anisimov, M. Farle, P. Poulopoulos, W. Platow, K. Baberschke, P. Isberg, R. Wäppling, A. M. N. Niklasson, and O. Eriksson, Phys. Rev. Lett. 82, 2390 (1999).

[150] R. D. McMichael and P. Krivosik, IEEE Trans. Magn. 40, 2 (2004).

[151] M. Farle, B. Mirwald-Schulz, A. N. Anisimov, W. Platow, and K. Baberschke, Phys. Rev. B 55, 3708 (1997).

[152] B. K. Kuanr, R. Camley, and Z. Celinski, J. Magn. Magn. Mater. 286, 276 (2005).

[153] O. Mosendz, B. Kardasz, D. Schmool, and B. Heinrich, J. Magn. Magn. Mater. 300, 174 (2006).

[154] Y. Ding, T. J. Klemmer, and T. M. Crawford, J. Appl. Phys. 96, 2969 (2004)

[155] D. Ghodgaonkar, V. Varadan, and V. Varadan, IEEE Trans. Instrum. Meas. 39, 387 (1990).

[156] H. Nemec, F. Kadlec, P. Kuzel, L. Duvillaret, and J.-L. Coutaz, Opt. Commun. 260, 175 (2006).

[157] S. S. Kalarickal, P. Krivosik, M. Wu, C. E. Patton, M. L. Schneider, P. Kabos, T. J. Silva, and J. P. Nibarger, J. Appl. Phys. 99, 093909 (2006).

[158] C.Bilzer, T. Devolder, P Crozat, and C. Chappert, IEEE Trans. Mag. 44, 3265 (2008)

[159] Y. Khivintsev, J. Marsh, V. Zagorodnii, I. Harward, J. Lovejoy, P. Krivosik, R. E. Camley, and Z. Celinski, Appl. Phys. Lett. 98, 042505 (2011).

[160] B. K. Kuanr, Y. V. Khivintsev, A. Hutchison, R. E. Camley, and Z. J. Celinski, IEEE Trans. Magnet. 43, 2645 (2007).

[161] Z. Lin and M. Kostylev, arXiv:1412.0777 (2014).

[162] R. W. Damon and H. van de Vaart, J. Appl. Phys. 36, 3453 (1965).

[163] C. S. Chang, M. Kostylev, E. Ivanov, J. Ding, and A. O. Adeyeye, Appl. Phys. Lett. 104, 032408 (2014).

[164] A. V. Chumak, P. Pirro, A. A. Serga, M. P. Kostylev, R. L. Stamps, H. Schultheiss, K. Vogt, S. J. Hermsdoerfer, B. Laegel, P. A. Beck, and B. Hillebrands, Appl. Phys. Lett. 95, 262508 (2009).

[165] Y. V. Khivintsev, L. Reisman, J. Lovejoy, R. Adam, C. M. Schneider, R. E. Camley, and Z. J. Celinski, J. Appl. Phys. 108, 023907 (2010).

[166] V. F. Dmitriev and B. A. Kalinikos, Sov. Phys. J. 31, 875 (1988).

[167] I. S. Maksymov and M. Kostylev, J. Phys. D: Appl. Phys. 46, 495001 (2013).

[168] V. E. Demidov, M. P. Kostylev, K. Rott, P. Krzysteczko, G. Reiss, and S. O. Demokritov, Appl. Phys. Lett. 95, 112509 (2009).

[169] M. Jamali, J. H. Kwon, S.-M. Seo, K.-J. Lee, and H. Yang, Scientific Reports 3, 3160 (2013).

[170] N. S. Almeida and D. L. Mills, Phys. Rev. B 53, 12232 (1996).

[171] M. Bailleul, Appl. Phys. Lett. 103, 192405 (2013).

[172] I. S. Maksymov, Z. Zhang, C. Chang, and M. Kostylev, IEEE Magnetics Letters, doi: 10.1109/LMAG.2014.2379721.

[173] A. Taflove and S. C. Hagness, Computational Electrodynamics: The Finite-Difference TimeDomain Method, 3rd ed., Artech House Publishers, Boston, 2005.

[174] M. Kostylev, J. Appl. Phys. 112, 093901 (2012).

[175] T. Kaneki, Electron. Lett. 5, 463 (1969).

[176] E. J. Denlinger, IEEE Trans. Microwave Theory Tech. 19, 30 (1971)

[177] M. Kostylev, J. Appl. Phys. 113, 053908 (2013).

[178] L. N. Hadley and D. M. Dennison, J. Opt. Soc. Am. 37, 451 (1947).

[179] S. Bauer, Am. J. Phys. 60, 257 (1992). 
[180] S. Fahy, C. Kittel, S. G. Louie, American Journal of Physics 56, 989 (1988).

[181] S. A Schelkunoff, Electromagnetic Waves, Van Nostrand, Princeton, NJ, 1943

[182] R. B. Schultz, V. C. Plantz, and D. R. Brush, IEEE Trans. Electromagn. Compat. 30, 187 (1988)

[183] I. S. Maksymov and M. Kostylev, J. Appl. Phys. 116, 173905 (2014).

[184] R. Bali, M. Kostylev, D. Tripathy, A. O. Adeyeye, and S. Samarin, Phys. Rev. B 85, 104414 (2012)

[185] V. E. Demidov, M. P. Kostylev, K. Rott, J. Münchenberger, G. Reiss, and S. O. Demokritov, Appl. Phys. Lett. 99, 082507 (2011).

[186] R. Magaraggia, M. Hambe, M. Kostylev, V. Nagarajan, and R. L. Stamps, Phys. Rev. B 84, 104441 (2011).

[187] Ngoc Chan, V. Kambersky, and D. Fraitová, J. Magn. Magn. Mater. 214, 93 (2000).

[188] W. E. Bailey, C. Cheng, R. Knut, O. Karis, S. Auffret, S. Zohar, D. Keavney, P. Warnicke, J.S. Lee, and D. A. Arena, Nature Commun. 4, 2025 (2013).

[189] J. W. Chen, D. M. Tang, B. S. Zhang, Y. Yang, M. Lu, and H. X. Lu, J. Phys.:Condens. Matter 19, 346227 (2007).

[190] M. N. O. Sadiku, Numerical Techniques in Electromagnetics, CRC Press, Boca Raton, 2000.

[191] V. Flovik, F. Macià, A. D. Kent, and E. Wahlström, arXiv:1412.1385 (2014).

[192] M. Kostylev, unpublished.

[193] R. F. Soohoo, J. Appl. Phys. 34, 1149 (1963).

[194] P. Krams, F. Lauks, R. L. Stamps, B. Hillebrands, and G. Guntherodt, Phys. Rev. Lett. 69, 3674 (1992).

[195] M. Kostylev, X. Wu, F. Altaf, P. Metaxas, M. Ali, D.Craig, R.L. Stamps, B. J. Hickey, and C. H. Marrows, unpublished

[196] W. Stoecklein, S. S. P. Parkin, and J. C. Scott, Phys. Rev. B. 38, 6847 (1988).

[197] J. Dho, X. Qi, H. Kim, J. L. MacManus-Driscoll, and M. G. Blamire, Adv. Mater. 18, 1445 (2006).

[198] L. W. Martin, Y.-H. Chu, M. B. Holcomb, M. Huijben, P. Yu, S.-J. Han, D. Lee, S. X. Wang, and R. Ramesh, Nano Lett. 8, 2050 (2008).

[199] S. M. Wu, S. A. Cybart, P. Yu, M. D. Rossell, J. X. Zhang, R. Ramesh, and R. C. Dynes, Nat. Mater. 9, 756 (2010).

[200] H. Ulrichs, V. E. Demidov, S. O. Demokritov, W. L. Lim, J. Melander, N. Ebrahim-Zadeh, and S. Urazhdin, Appl. Phys. Lett. 102, 132402 (2013).

[201] B. F. Miao, S. Y. Huang, D. Qu, and C. L. Chien, Phys. Rev. Lett. 111, 066602 (2013).

[202] M. Harder, Z. X. Cao, Y. S. Gui, X. L. Fan, and C.-M. Hu, Phys. Rev. B 84, 054423 (2011).

[203] I. S. Maksymov and M. Kostylev J. Appl. Phys. 113, 043927 (2013).

[204] "Artificial crystals and metamaterials for spin waves from nanopatterned $\mathrm{Ni}_{80} \mathrm{Fe}_{20}$ antidot lattices", Chapter 14 (pp. 191 - 203) in Topics in Applied Physics: Magnonics, S.O. Demokritov, A.N. Slavin (Eds), Springer (2013).

[205] M. Krawczyk and D. Grundler, J. Phys.: Condens. Matter. 26, 123202 (2014).

[206] F. Aliev, A. Awad, D. Dieleman, A. Lara, V. Metlushko, and K.Y. Guslienko, Phys. Rev. B 84, 144406 (2011).

[207] J. Ding, M. Kostylev, and A. O. Adeyeye, Phys. Rev. B 84, 054425 (2011).

[208] J. Ding and A. O. Adeyeye, Appl. Phys. Lett. 101, 103117 (2012).

[209] X. M. Liu, J. Ding, G. N. Kakazei, and A. O. Adeyeye, Appl. Phys. Lett. 103, 062401 (2013).

[210] A. A. Awad, G. R. Aranda, D. Dieleman, K. Y. Guslienko, G. N. Kakazei, B. A. Ivanov, and F. 
G. Aliev, Appl. Phys. Lett. 97, 132501 (2010).

[211] G. N. Kakazei, X. M. Liu, J. Ding1 and A. O. Adeyeye, Appl. Phys. Lett. 104, 042403 (2014).

[212] K. Yu. Guslienko, S. O. Demokritov, B. Hillebrands, and A. N. Slavin, Phys. Rev. B 66, 132402 (2002).

[213] C. Mathieu, J. Jorzick, A. Frank, S. O. Demokritov, A. N. Slavin, B. Hillebrands, Phys. Rev. Lett. 81, 3968 (1998).

[214] A. O. Adeyeye and N. Singh, J. Phys. D: Appl. Phys. 41, 153001 (2008).

[215] L. Willig, Magnetisation Dynamics and Magneto-Optics of Nanostructures, Master Thesis, School of Physics, University of Western Australia (2014).

[216] J. Ding, M. Kostylev, and A. O. Adeyeye, Phys. Rev. Lett. 107, 047205 (2011).

[217] V. E. Demidov, S. Urazhdin, E. R. J. Edwards, M. D. Stiles, R. D. McMichael, and S. O. Demokritov, Phys. Rev. Lett. 107, 107204 (2011).

[218] B. Bhoi, T. Cliff, I. S. Maksymov, M. Kostylev, R. Aiyar, N. Venkataramani, S. Prasad, and R. L. Stamps, J. Appl. Phys., DOI: 10.1063/1.4904857.

[219] Y. Kobljanskyj, G. Melkov, K. Guslienko, V. Novosad, S. D. Bader, M. Kostylev, and A. Slavin, Scientific Reports 2, 478 (2012).

[220] M. Lapine, I. V. Shadrivov, and Yu. S. Kivshar, Rev. Mod. Phys. 86, 1093 (2014).

[221] O. Klein, V. Charbois, V. V. Naletov, and C. Fermon, Phys. Rev. B 67, 220407(R) (2003)

[222] V. V. Naletov, G. de Loubens, V. Charbois, O. Klein, V. S. Tiberkevich, and A. N. Slavin, Phys Rev. B 75, 140405(R) (2007).

[223] G. de Loubens, V. V. Naletov, O. Klein, J. Ben Youssef, F. Boust, and N. Vukadinovic, Phys. Rev. Lett. 98, 127601 (2007).

[224] E. Nazaretski, T. Mewes, D. V. Pelekhov, P. C. Hammel and R. Movshovich, AIP Conf. Proc. 850, 1641 (2006).

[225] E. Nazaretski, I. Martin, R. Movshovich, D. V. Pelekhov, P. C. Hammel, M. Zalalutdinov, J. W. Baldwin, B. Houston, and T. Mewes, Appl. Phys. Lett. 90, 234105 (2007).

[226] E. Nazaretski, J. D. Thompson, D. V. Pelekhov, T. Mewes, P. E. Wigen, J. Kim, M. Zalalutdinov, J. W. Baldwin, B. Houston, P. C. Hammel, and R. Movshovich, J. Magn. Magn. Mater. 310, e941 (2007).

[227] V. E. Demidov, S. Urazhdin, and S. O. Demokritov, Nature Materials 9, 984 (2010).

[228] V. E. Demidov, S. Urazhdin, E. R. J. Edwards, and S. O. Demokritov, Appl. Phys. Lett. 99, $172501(2011)$

[229] C. W. Sandweg, Y. Kajiwara, A. V. Chumak, A. A. Serga, V. I. Vasyuchka, M. B. Jungfleisch, E. Saitoh, and B. Hillebrands, Phys. Rev. Lett. 106, 216601 (2011).

[230] C. Hahn, G. de Loubens, O. Klein, M. Viret, V. V. Naletov, and J. Ben Youssef, Phys. Rev. B 87, 174417 (2013).

[231] O. Mosendz, V. Vlaminck, J. E. Pearson, F. Y. Fradin, G. E. W. Bauer, S. D. Bader, and A. Hoffmann, Phys. Rev. B 82, 214403 (2010).

[232] Y. Sun, H. Chang, M. Kabatek, Y.-Y. Song, Z. Wang, M. Jantz, W. Schneider, and M. Wu, Phys. Rev. Lett. 111, 106601 (2013)

[233] C. Nistor, K. Sun, Z. Wang, M. Wu, C. Mathieu, and M. Hadley, Appl. Phys. Lett. 95, 012504 (2009).

[234] Z. Wang, Y.-Y. Song, Y. Sun, J. Bevivino, M. Wu, V. Veerakumar, T. J. Fal, and R. E. Camley, Appl. Phys. Lett. 97, 072509 (2010).

[235] Z. Wang, Y. Sun, M. Wu, V. Tiberkevich, and A. Slavin, Phys. Rev. Lett. 107, 146602 (2011).

[236] J. E. Lezac, P. Quéffélec, and A. Chevalier, IEEE Trans. Magnet. 46, 1687 (2010). 
[237] S. T. B. Goennenwein, S. W. Schink, A. Brandlmaier, A. Boger, M. Opel, R. Gross, R. S. Keizer, T. M. Klapwijk, A. Gupta, H. Huebl, C. Bihler, and M. S. Brandt, Appl. Phys. Lett. 90, 162507 (2007).

[238] B. Obry, V. I. Vasyuchka, A. V. Chumak, A. A. Serga, and B. Hillebrands, Appl. Phys. Lett. 101, 192406 (2012)

[239] L. A. Harris, J. S. Riffle, and T. G. St. Pierre, J. Australian Ceramic Society 41, 23 (2005).

[240] S.-H. Chung, A. Hoffmann, K. Guslienko, S. D. Bader, C. Liu, B. Kay, L. Makowski, and L. Chen, J. Appl. Phys. 97, 10R101 (2005).

[241] S.-H. Chung, A. Hoffmann, L. Chen, S. Sun, K. Guslienko, M. Grimsditch, and S. D. Bader, Journal of Magnetics (Korean Magnetic Society) 11, 189 (2006). 